\title{
Spectral gaps of Schrödinger operators with periodic singular potentials
}

\author{
Plamen Djakov and Boris Mityagin \\ Communicated by Charles Li, received March 27, 2009.
}

\begin{abstract}
By using quasi-derivatives we develop a Fourier method for studying the spectral gaps of one dimensional Schrödinger operators with periodic singular potentials $v$. Our results reveal a close relationship between smoothness of potentials and spectral gap asymptotics under a priori assumption $v \in H_{\text {loc }}^{-1}(\mathbb{R})$. They extend and strengthen similar results proved in the classical case $v \in L_{\text {loc }}^{2}(\mathbb{R})$.
\end{abstract}

\section{Contents}

1. Inroduction $\quad 95$

2. Preliminaries $\quad 99$

3. Basic equation 104

4. Estimates of $\alpha(v ; n, z)$ and $\beta^{ \pm}(v ; n, z) \quad 109$

5. Estimates for $\gamma_{n}$. 117

6. Main results for real-valued potentials 121

7. Complex-valued $H^{-1}$-potentials 127

8. Comments 133

9. Appendix:Deviations of Riesz projections of Hill operators with singular potentials 143

$\begin{array}{ll}\text { References } & 162\end{array}$

\section{Inroduction}

We consider the Hill operator

$$
L y=-y^{\prime \prime}+v(x) y, \quad x \in I=[0, \pi],
$$

1991 Mathematics Subject Classification. 34L40, 47E05, 34B30.

Key words and phrases. Schrödinger operator, singular periodic potential, spectral gaps.

B. Mityagin acknowledges the support of the Scientific and Technological Research Council of Turkey and the hospitality of Sabanci University, Istanbul, in April-June, 2008. 
with a singular complex-valued $\pi$-periodic potential $v \in H_{l o c}^{-1}(\mathbb{R})$, i.e.,

$$
v(x)=v_{0}+Q^{\prime}(x), \quad Q \in L_{l o c}^{2}(\mathbb{R}), \quad Q(x+\pi)=Q(x),
$$

with $Q$ having zero mean

$$
q(0)=\int_{0}^{\pi} Q(x) d x=0
$$

then

$$
Q(x)=\sum_{m \in 2 \mathbb{Z} \backslash\{0\}} q(m) e^{i m x}, \quad\|Q\|_{L^{2}(I)}^{2}=\|q\|^{2}=\sum_{m \in 2 \mathbb{Z} \backslash\{0\}}|q(m)|^{2}<\infty,
$$

where $q=(q(m))_{m \in 2 \mathbb{Z} \text {. }}$

Analysis of the Hill or Sturm-Liouville operators, or their multi-dimensional analogues $-\Delta+v(x)$ with point (surface) interaction ( $\delta$-type) potentials has a long history. From the early 1960's (F. Berezin, L. Faddeev, R. Minlos [6, 7, 57]) to around 2000 the topic has been studied in detail; see books [2, 3] and references there. For specific potentials see W. N. Everitt and A. Zettl [23, 24] and P. Kurasov [51].

A more general approach which allows to consider any singular potential (beyond $\delta$-functions or Coulomb type) in negative Sobolev spaces has been initiated by A. Shkalikov and his coauthors Dzh. Bak, M. Nel̆man-zade and A. Savchuk $[\mathbf{5}, \mathbf{6 2}, \mathbf{6 3}, \mathbf{6 9}]$. It led to the spectral theory of Sturm-Liouville operators with distribution potentials developed by A. Savchuk and A. Shkalikov $[\mathbf{6 8}, \mathbf{7 0}, \mathbf{7 1}, \mathbf{7 2}]$, and R. Hryniv and Ya. Mykytyuk $[34,35,36,37,38,39])$.

Another approach to the study of the Sturm-Liouville operators with nonclassical potentials comes from M. Krein $[\mathbf{4 8 , 4 9 ]}$. E. Korotyaev (see $[\mathbf{4 5}, \mathbf{4 6}, \mathbf{4 7}]$ and the references therein) uses this approach very successfully but it seems to be limited to the case of real potentials.

A. Savchuk and A. Shkalikov [71] consider a broad class of boundary conditions (bc) - see Formula (1.6) in Theorem 1.5 there - in terms of a function $y$ and its quasi-derivative

$$
u=y^{\prime}-Q y .
$$

In particular, the proper form of periodic $\mathrm{Per}^{+}$and antiperiodic $\left(\mathrm{Per}^{-}\right) b c$ is

$$
\operatorname{Per}^{ \pm}: \quad y(\pi)= \pm y(0), \quad u(\pi)= \pm u(0) .
$$

If the potential $v$ happens to be an $L^{2}$-function those $b c$ are identical to the classical ones (see discussion in [16], Section 6.2).

The Dirichlet Dir bc is more simple:

$$
\text { Dir : } \quad y(0)=0, \quad y(\pi)=0
$$

it does not require quasi-derivatives, so it is defined in the same way as for $L^{2}-$ potentials.

In our analysis of instability zones of the Hill and Dirac operators (see [14] and the comments there) we follow an approach $([\mathbf{4 0}, \mathbf{4 1}, \mathbf{9}, \mathbf{1 1}, \mathbf{6 0}, \mathbf{1 2}])$ based on Fourier Method. But in the case of singular potentials it may happen that the functions

$$
u_{k}=e^{i k x} \quad \text { or } \quad \sin k x, \quad k \in \mathbb{Z}
$$


have their $L_{b c}$-images outside $L^{2}$. Moreover, for some singular potentials $v$ we have $L_{b c} f \notin L^{2}$ for any nonzero smooth (say $C^{2}$-function) $f$. (For example, choose

$$
v(x)=\sum_{r} a(r) \delta_{*}(x-r), \quad r \text { rational, } r \in I,
$$

with $a(r)>0, \sum_{r} a(r)=1$ and $\delta_{*}(x)=\sum_{k \in \mathbb{Z}} \delta(x-k \pi)$.) This implies, for any reasonable $b c$, that the eigenfunctions $\left\{u_{k}\right\}$ of the free operator $L_{b c}^{0}$ are not necessarily in the domain of $L_{b c}$.

Yet, in $[\mathbf{1 5}, \mathbf{1 6}]$ we gave a justification of the Fourier method ${ }^{1}$ for operators $L_{b c}$ with $H^{-1}$-potentials and $b c=P e r^{ \pm}$or Dir. Our results are announced in [15], and all technical details of justification of the Fourier method are provided in [16]. In Section 2 we remind our constructions from [16] which is essentially a general introduction to the present paper. A proper understanding of the boundary conditions $\left(\mathrm{Per}^{ \pm}\right.$or Dir) - see (1.5) and the formulas $(2.1),\left(a^{*}\right),\left(b^{*}\right)$ below - and careful definitions of the corresponding operators and their domains are provided by using quasi-derivatives. To great extend we follow the approach suggested and developed by A. Savchuk and A. Shkalikov $[\mathbf{6 9}, \mathbf{7 1}]$ (see also $[\mathbf{7 0}, \mathbf{7 2}]$ ) and further, by R. Hryniv and Ya. Mykytyuk [34] and [37, 39].

The Hill-Schrödinger operator $L$ with a singular potential $v \in H^{-1}$ has, for each $n \geq n_{0}(v)$ in the disc of center $n^{2}$ and radius $n$, one Dirichlet eigenvalue $\mu_{n}$ and two (counted with their algebraic multiplicity) periodic (if $n$ is even) or antiperiodic (if $n$ is odd) eigenvalues $\lambda_{n}^{-}, \lambda_{n}^{+}$(see Proposition 4 below, or Theorem 21 in $[\mathbf{1 6}])$.

Our main goal in the present paper is to study, for singular potentials $v \in H^{-1}$, the relationship between the smoothness of $v$ and the asymptotic behavior of spectral gaps $\gamma_{n}=\left|\lambda_{n}^{+}-\lambda_{n}^{-}\right|$and deviations $\delta_{n}=\left|\mu_{n}-\left(\lambda_{n}^{+}+\lambda_{n}^{-}\right) / 2\right|$. In the classical case $v \in L^{2}$ this relationship means, roughly speaking, that the sequences $\left(\gamma_{n}\right)$ and $\left(\delta_{n}\right)$ decay faster if the potential is smoother, and vise versa. Of course, to make this statement precise one needs to consider appropriate classes of smooth functions and related classes of sequences.

This phenomenon was discovered by H. Hochstadt [32, 33], who showed for real-valued potentials $v \in L^{2}$ the following connection between the smoothness of $v$ and the rate of decay of spectral gaps (or, the lengths of instability zones) $\gamma_{n}=\lambda_{n}^{+}-\lambda_{n}^{-}:$If

(i) $v \in C^{\infty}$, i.e., $v$ is infinitely differentiable, then

(ii) $\gamma_{n}$ decreases more rapidly than any power of $1 / n$.

If a continuous function $v$ is a finite-zone potential, i.e., $\gamma_{n}=0$ for all large enough $n$, then $v \in C^{\infty}$.

In the middle of 70's (see [53], [54]) the latter statement was extended, namely, it was shown, for real $L^{2}([0, \pi])$-potentials $v$, that a power decay of spectral gaps implies infinite differentiability, i.e., the implication $(i i) \Rightarrow(i)$ holds.

\footnotetext{
${ }^{1}$ Maybe it is worth to mention that T. Kappeler and C. Möhr [42] analyze "periodic and Dirichlet eigenvalues of Schrödinger operators with singular potential" but they never tell how these operators (or boundary conditions) are defined on the interval, i.e., in a Hilbert space $L^{2}([0, \pi])$. At some point they jump without any justification or explanation into weighted $\ell^{2}-$ sequence spaces (an analog of Sobolev spaces $H^{a}$ ) and consider the same sequence space operators that appear in the regular case, i.e., if $v \in L_{\text {per }}^{2}(\mathbb{R})$. But without formulating which Sturm-Liouville problem is considered, what are the corresponding boundary conditions, what is the domain of the operator, etc., it is not possible to pass from a non-defined differential operator to its Fourier representation.
} 
E. Trubowitz [76] has used the Gelfand-Levitan [26] trace formula and the Dubrovin equations $[\mathbf{2 0 , 2 1}]$ to explain, that a real $L^{2}([0, \pi])$-potential $v(x)=$ $\sum_{k \in \mathbb{Z}} V(2 k) \exp (2 i k x)$ is analytic, i.e.,

$$
\exists A>0: \quad|V(2 k)| \leq M e^{-A|k|},
$$

if and only if the spectral gaps decay exponentially, i.e.,

$$
\exists a>0: \quad \gamma_{n} \leq C e^{-a|k|}
$$

M. Gasymov [25] showed that if a potential $v \in L^{2}([0, \pi])$ has the form $v(x)=$ $\sum_{k=0}^{\infty} v_{k} \exp (2 i k x)$ then $\gamma_{n}=0 \forall n$. Therefore, in general the decay of $\gamma_{n}$ cannot give any restriction on the smoothness of complex potentials. V. Tkachenko $[\mathbf{7 4}, \mathbf{7 5}]$ suggested to bring the deviations $\delta_{n}$ into consideration. As a further development, J.-J. Sansuc and V. Tkachenko [67] gave a statement of Hochstadt type: A potential $v \in L^{2}([0, \pi])$ belongs to the Sobolev space $H^{m}, m \in \mathbb{N}$ if and only if

$$
\sum\left(\left|\gamma_{n}\right|^{2}+\left|\delta_{n}\right|^{2}\right)\left(1+n^{2 m}\right)<\infty
$$

When talking about general classes of $\pi$-periodic smooth functions, we characterize the smoothness by a weight $\Omega=(\Omega(n))$, and consider the "Sobolev" space

$$
H(\Omega)=\left\{v(x)=\sum_{k \in \mathbb{Z}} v_{k} e^{2 i k x}, \quad \sum_{k \in \mathbb{Z}}\left|v_{k}\right|^{2}(\Omega(k))^{2}<\infty\right\} .
$$

The related sequence space is determined as

$$
\ell_{\Omega}^{2}=\left\{\xi=\left(\xi_{n}\right)_{0}^{\infty}: \sum_{n \in \mathbb{N}}\left|\xi_{n}\right|^{2}(\Omega(n))^{2}<\infty\right\} .
$$

In this terminology and under a priori assumption $v \in L^{2}$, one may consider the following general question on the relationship between the potential smoothness and decay rate of spectral gaps and deviations: Is it true that the following conditions $(A)$ and $(B)$ are equivalent:

$$
\text { (A) } v \in H(\Omega) ; \quad(B) \gamma \in \ell^{2}(\Omega) \text { and } \delta \in \ell^{2}(\Omega)
$$

The answer is positive for weights $\Omega$ in a broad range of growths from being constant to growing exponentially - see more detailed discussion and further results in $[\mathbf{1 4}]$, in particular Theorems 54 and 67.

Let us note that in the classical case $v \in L^{2}$ we have $v_{k} \rightarrow 0$ as $|k| \rightarrow \infty$, and $\gamma_{n} \rightarrow 0, \delta_{n} \rightarrow 0$ as $n \rightarrow \infty$. Therefore, if $v \in L^{2}$ then we consider weights which satisfy the condition

$$
\inf _{n} \Omega(n)>0
$$

In the case $v \in H^{-1}$ the sequences $\left(v_{k}\right),\left(\gamma_{n}\right),\left(\delta_{n}\right)$ may not converge to zero, but $v_{k} / k \rightarrow 0$ as $|k| \rightarrow \infty$, and $\gamma_{n} / n \rightarrow 0, \delta_{n} / n \rightarrow 0$ as $n \rightarrow \infty$. Therefore, in the case $v \in H^{-1}$ it is natural to consider weights which satisfy

$$
\inf _{n} n \Omega(n)>0 \text {. }
$$

The main results of the present paper assert, under a priori assumption $v \in H^{-1}$, that the conditions (A) and (B) above are equivalent if $\Omega$ satisfies (1.10) and some 
other mild restrictions (see for precise formulations Theorem 28 and Theorem 29). Since the condition (1.9) is more restrictive than (1.10), these results extend and strengthen our previous results in the classical case $v \in L^{2}$ as well.

Sections 3-7, step by step, lead us to the proofs of Theorems 28 and 29. As before in the regular case $v \in L^{2}([\mathbf{1 1}]$, Proposition 4) an important ingredient of the proof (see in particular Section 7) is the following assertion about the deviations of Riesz projections $P_{n}-P_{n}^{0}$ of Hill operators (with singular potentials) $L_{b c}$ and free operators $L_{b c}^{0}$ :

$$
\left\|P_{n}-P_{n}^{0}\right\|_{L^{1} \rightarrow L^{\infty}} \rightarrow 0 .
$$

This fact is important not only in the context of Theorem 29 but also for a series of results on convergence of spectral decompositions, both in the case of Hill operators with singular potentials and 1D Dirac operators with periodic $L^{2}$-potentials (see $[\mathbf{1 7}, \mathbf{1 8}, \mathbf{1 9}])$. The proof and analysis of the statement (1.11) is put aside of the main text as Appendix, Section 9.

Section 8 gives a few comments (in historical context) on different parts of the general scheme and its realization. In particular, we remind and extend in Proposition 38 the observation of J. Meixner and F. W. Schäfke [55, 56] that the Dirichlet eigenvalues of the Hill-Mathieu operator could be analytic only in a bounded disc. They gave upper bounds of the radii of these discs (see Satz 8, Section 1.5 in [55] and p. 87, the last paragraph, in [56]). This is an interesting topic of its own (see [78]-[82] and [13]). This analysis has been extended to families of tri-diagonal matrix operators in $[\mathbf{1}]$.

\section{Preliminaries}

It is known (e.g., see [34], Remark 2.3) that every $\pi$-periodic potential $v \in$ $H_{l o c}^{-1}(\mathbb{R})$ has the form

$$
v=C+Q^{\prime}, \quad \text { where } C=\text { const }, Q \text { is } \pi \text { - periodic, } \quad Q \in L_{\text {loc }}^{2}(\mathbb{R}) .
$$

Therefore, formally we have

$$
-y^{\prime \prime}+v \cdot y=\ell(y):=-\left(y^{\prime}-Q y\right)^{\prime}-Q\left(y^{\prime}-Q y\right)+\left(C-Q^{2}\right) y .
$$

So, one may introduce the quasi-derivative $u=y^{\prime}-Q y$ and replace the distribution equation $-y^{\prime \prime}+v y=0$ by the following system of two linear differential equations with coefficients in $L_{l o c}^{1}(\mathbb{R})$

$$
y^{\prime}=Q y+u, \quad u^{\prime}=\left(C-Q^{2}\right) y-Q u .
$$

By the Existence-Uniqueness Theorem for systems of linear o.d.e. with $L_{l o c}^{1}(\mathbb{R})-$ coefficients (e.g., see $[\mathbf{4}, \mathbf{6 1}]$ ), the Cauchy initial value problem for the system (2.1) has, for each pair of numbers $(a, b)$, a unique solution $(y, u)$ such that $y(0)=$ $a, u(0)=b$. This makes possible to apply the Floquet theory to the system (2.1), to define a Lyapunov function, etc.

We define the Schrödinger operator $L(v)$ in the domain

$$
D(L(v))=\left\{y \in H^{1}(\mathbb{R}): \quad y^{\prime}-Q y \in L^{2}(\mathbb{R}) \cap W_{1, l o c}^{1}(\mathbb{R}), \quad \ell(y) \in L^{2}(\mathbb{R})\right\},
$$

$$
L(v) y=\ell(y)=-\left(y^{\prime}-Q y\right)^{\prime}-Q y^{\prime} .
$$


The domain $D(L(v))$ is dense in $L^{2}(\mathbb{R})$, the operator $L(v)$ is a closed, and its spectrum could be described in terms of the corresponding Lyapunov function (see Theorem 4 in [16]).

In the classical case $v \in L_{l o c}^{2}(\mathbb{R})$, if $v$ is a real-valued then by the FloquetLyapunov theory (see $[\mathbf{2 2}, \mathbf{5 0}, \mathbf{5 2}, \mathbf{8 3}]$ ) the spectrum of $L(v)$ is absolutely continuous and has a band-gap structure, i.e., it is a union of closed intervals separated by spectral gaps

$$
\left(-\infty, \lambda_{0}\right),\left(\lambda_{1}^{-}, \lambda_{1}^{+}\right),\left(\lambda_{2}^{-}, \lambda_{2}^{+}\right), \ldots,\left(\lambda_{n}^{-}, \lambda_{n}^{+}\right), \ldots
$$

The points $\left(\lambda_{n}^{ \pm}\right)$are defined by the spectra of the corresponding Hill operator considered on the interval $[0, \pi]$, respectively, with periodic (for even $n$ ) and antiperiodic (for odd $n$ ) boundary conditions $(b c)$ :

(a) periodic $\operatorname{Per}^{+}: \quad y(\pi)=y(0), y^{\prime}(\pi)=y^{\prime}(0)$;

(b) antiperiodic $P e r^{-}$: $\quad y(\pi)=-y(0), y^{\prime}(\pi)=-y^{\prime}(0)$;

Recently a similar statement was proved in the case of real singular potentials $v \in H^{-1}$ by R. Hryniv and Ya. Mykytyuk [34].

Following A. Savchuk and A. Shkalikov [69, 71], let us consider (in the case of singular potentials) periodic and anti-periodic boundary conditions $\mathrm{Per}^{ \pm}$of the form

$\left(a^{*}\right) \quad P e r^{+}: \quad y(\pi)=y(0),\left(y^{\prime}-Q y\right)(\pi)=\left(y^{\prime}-Q y\right)(0)$.

$\left(b^{*}\right) \quad P_{e r^{-}:} \quad y(\pi)=-y(0),\left(y^{\prime}-Q y\right)(\pi)=-\left(y^{\prime}-Q y\right)(0)$.

R. Hryniv and Ya. Mykytyuk [34] showed, that the Floquet theory for the system (2.1) could be used to explain that if the potential $v \in H_{l o c}^{-1}$ is real-valued, then $L(v)$ is a self-adjoint operator having absolutely continuous spectrum with band-gap structure, and the spectral gaps are determined by the spectra of the corresponding Hill operators $L_{P e r^{ \pm}}$defined on $[0, \pi]$ by $L_{P e r^{ \pm}}(y)=\ell(y)$ for $y \in$ $D\left(L_{P e r^{ \pm}}\right)$, where

$$
D\left(L_{P e r^{ \pm}}\right)=\left\{y \in H^{1}: y^{\prime}-Q y \in W_{1}^{1}([0, \pi]), \quad\left(a^{*}\right) \text { or }\left(b^{*}\right) \text { holds, } \ell(y) \in H^{0}\right\} .
$$

(Hereafter the short notations $H^{1}=H^{1}([0, \pi]), \quad H^{0}=L^{2}([0, \pi])$ are used.)

We set

$$
H_{P e r^{ \pm}}^{1}=\left\{f \in H^{1}: f(\pi)= \pm f(0)\right\}, H_{D i r}^{1}=\left\{f \in H^{1}: f(\pi)=f(0)=0\right\} .
$$

One can easily see that $\left\{u_{k}=e^{i k x}, k \in \Gamma_{P_{e r}+}=2 \mathbb{Z}\right\}$ is an orthogonal basis in $H_{P e r^{+}}^{1},\left\{u_{k}=e^{i k x}, k \in \Gamma_{P_{e r^{-}}}=1+2 \mathbb{Z}\right\}$ is an orthogonal basis in $H_{P e r^{-}}^{1}$, and $\left\{u_{k}=\sqrt{2} \sin k x, k \in \Gamma_{D i r}=\mathbb{N}\right\}$ is an orthogonal basis in $H_{D i r}^{1}$. From here it follows, for $b c=P e r^{ \pm}$or Dir, that

$$
H_{b c}^{1}=\left\{f(x)=\sum_{k \in \Gamma_{b c}} f_{k} u_{k}(x): \quad\|f\|_{H^{1}}^{2}=\sum_{k \in \Gamma_{b c}}\left(1+k^{2}\right)\left|f_{k}\right|^{2}<\infty\right\} .
$$

Now, we are ready to explain the Fourier method for studying the spectra of the operators $L_{P e r^{ \pm}}$. We set

$$
V(k)=i k q(k), \quad k \in 2 \mathbb{Z},
$$

where $q(k)$ are the Fourier coefficients of $Q$ defined in (1.4).

Let $\mathcal{F}: H^{0} \rightarrow \ell^{2}\left(\Gamma_{P e r^{ \pm}}\right)$be the Fourier isomorphism defined by mapping a function $f \in H^{0}$ to the sequence $\left(f_{k}\right)$ of its Fourier coefficients $f_{k}=\left(f, u_{k}\right)$, where $\left\{u_{k}, k \in \Gamma_{P e r^{ \pm}}\right\}$is the corresponding basis introduced above. Let $\mathcal{F}^{-1}$ be the inverse Fourier isomorphism. 
Consider the operators $\mathcal{L}_{+}$and $\mathcal{L}_{-}$acting as

$$
\mathcal{L}_{ \pm}(z)=\left(h_{k}(z)\right)_{k \in \Gamma_{P e r} \pm}, h_{k}(z)=k^{2} z_{k}+\sum_{m \in \Gamma_{P e r} \pm} V(k-m) z_{m}+C z_{k},
$$

respectively, in

$$
D\left(\mathcal{L}_{ \pm}\right)=\left\{z \in \ell^{2}\left(|k|, \Gamma_{\text {Per }}\right): \mathcal{L}_{ \pm}(z) \in \ell^{2}\left(\Gamma_{P e r^{ \pm}}\right)\right\}
$$

where

$$
\ell^{2}\left(|k|, \Gamma_{P e r^{ \pm}}\right)=\left\{z=\left(z_{k}\right)_{k \in \Gamma_{P e r^{ \pm}}}: \sum_{k}\left(1+|k|^{2}\right)\left|z_{k}\right|^{2}<\infty\right\} .
$$

Proposition 1. (Theorem 11 in [16]) In the above notations, we have

$$
D\left(L_{P e r^{ \pm}}\right)=\mathcal{F}^{-1}\left(D\left(\mathcal{L}_{ \pm}\right)\right), \quad L_{P e r \pm}=\mathcal{F}^{-1} \circ \mathcal{L}_{ \pm} \circ \mathcal{F} .
$$

Next we study the Hill-Schrödinger operator $L_{D i r}(v), v=C+Q^{\prime}$, generated by the differential expression $\ell_{Q}(y)$ when considered with Dirichlet boundary conditions Dir : $y(0)=y(\pi)=0$. We set

$$
L_{D i r}(v) y=\ell_{Q}(y), \quad y \in D\left(L_{D i r}(v)\right)
$$

where

$$
D\left(L_{D i r}\right)=\left\{y \in H^{1}: y^{\prime}-Q y \in W_{1}^{1}([0, \pi]), y(0)=y(\pi)=0, \ell_{Q}(y) \in H^{0}\right\} .
$$

Proposition 2. (Theorem 13 in $[\mathbf{1 6}]$ ) Suppose $v \in H_{l o c}^{-1}(\mathbb{R})$ is $\pi$-periodic. Then:

(a) the domain $D\left(L_{D i r}(v)\right)$ is dense in $H^{0}$;

(b) the operator $L_{D i r}(v)$ is closed and its conjugate operator is

$$
\left(L_{\operatorname{Dir}}(v)\right)^{*}=L_{\operatorname{Dir}}(\bar{v}),
$$

so, in particular, if $v$ is real, then the operator $L_{D i r}(v)$ is self-adjoint;

(c) the spectrum $S p\left(L_{D i r}(v)\right)$ of the operator $L_{D i r}(v)$ is discrete, and

$$
S p\left(L_{D i r}(v)\right)=\left\{\lambda \in \mathbb{C}: y_{2}(\pi, \lambda)=0\right\} .
$$

Let

$$
Q(x)=\sum_{k \in \mathbb{N}} \tilde{q}(k) \sqrt{2} \sin k x
$$

be the sine Fourier series of $Q$. We set

$$
\tilde{V}(k)=k \tilde{q}(k), \quad k \in \mathbb{N} .
$$

Let $\mathcal{F}: H^{0} \rightarrow \ell^{2}(\mathbb{N})$ be the Fourier isomorphisms that maps a function $f \in H^{0}$ to the sequence $\left(f_{k}\right)_{k \in \mathbb{N}}$ of its Fourier coefficients $f_{k}=(f, \sqrt{2} \sin k x)$, and let $\mathcal{F}^{-1}$ be the inverse Fourier isomorphism.

We set, for each $z=\left(z_{k}\right) \in \ell^{2}(\mathbb{N})$,

$$
h_{k}(z)=k^{2} z_{k}+\frac{1}{\sqrt{2}} \sum_{m \in \mathbb{N}}(\tilde{V}(|k-m|)-\tilde{V}(k+m)) z_{m}+C z_{k},
$$

and consider the operator $\mathcal{L}_{d}$ defined by

in the domain

$$
\mathcal{L}_{d}(z)=\left(h_{k}(z)\right)_{k \in \mathbb{N}}
$$

$$
D\left(\mathcal{L}_{d}\right)=\left\{z \in \ell^{2}(|k|, \mathbb{N}): \quad \mathcal{L}_{d}(z) \in \ell^{2}(\mathbb{N})\right\}
$$


where

$$
\ell^{2}(|k|, \mathbb{N})=\left\{z=\left(z_{k}\right)_{k \in \mathbb{N}}: \sum_{k}|k|^{2}\left|z_{k}\right|^{2}<\infty\right\} .
$$

Proposition 3. (Theorem 16 in [16]) In the above notations, we have

$$
D\left(L_{\text {Dir }}\right)=\mathcal{F}^{-1}\left(D\left(\mathcal{L}_{d}\right)\right), \quad L_{\text {Dir }}=\mathcal{F}^{-1} \circ \mathcal{L}_{d} \circ \mathcal{F} .
$$

Let $L^{0}$ be the free operator, and let $V$ denotes the operator of multiplication by $v$. One of the technical difficulties that arises for singular potentials is connected with the standard perturbation type formulae for the resolvent $R_{\lambda}=\left(\lambda-L^{0}-V\right)^{-1}$. In the case where $v \in L^{2}([0, \pi])$ one can represent the resolvent in the form

$R_{\lambda}=\left(1-R_{\lambda}^{0} V\right)^{-1} R_{\lambda}^{0}=\sum_{k=0}^{\infty}\left(R_{\lambda}^{0} V\right)^{k} R_{\lambda}^{0}$ or $R_{\lambda}=R_{\lambda}^{0}\left(1-V R_{\lambda}^{0}\right)^{-1}=\sum_{k=0}^{\infty} R_{\lambda}^{0}\left(V R_{\lambda}^{0}\right)^{k}$,

where $R_{\lambda}^{0}=\left(1-L^{0}\right)^{-1}$. The simplest conditions that guarantee the convergence of these series are

$$
\left\|R_{\lambda}^{0} V\right\|<1, \quad \text { respectively, } \quad\left\|V R_{\lambda}^{0}\right\|<1 .
$$

Each of these conditions can be easily verified for large enough $n$ if $\operatorname{Re} \lambda \in[n-$ $1, n+1]$ and $\left|\lambda-n^{2}\right| \geq C\left(\|v\|_{L^{2}}\right)$, which leads to a series of results on the spectra, zones of instability and spectral decompositions.

The situation is more complicated if $v$ is a singular potential. Then, in general, there are no good estimates for the norms of $R_{\lambda}^{0} V$ and $V R_{\lambda}^{0}$. However, one can write $R_{\lambda}$ in the form

$$
R_{\lambda}=R_{\lambda}^{0}+R_{\lambda}^{0} V R_{\lambda}^{0}+R_{\lambda}^{0} V R_{\lambda}^{0} V R_{\lambda}^{0}+\cdots=K_{\lambda}^{2}+\sum_{m=1}^{\infty} K_{\lambda}\left(K_{\lambda} V K_{\lambda}\right)^{m} K_{\lambda},
$$

provided $\left(K_{\lambda}\right)^{2}=R_{\lambda}^{0}$. We define an operator $K=K_{\lambda}$ with this property by its matrix representation

$$
K_{j m}=\left(\lambda-j^{2}\right)^{-1 / 2} \delta_{j m}, \quad j, m \in \Gamma_{b c},
$$

where $z^{1 / 2}=\sqrt{r} e^{i \varphi / 2} \quad$ if $z=r e^{i \varphi},-\pi \leq \varphi<\pi$. Then $R_{\lambda}$ is well-defined if

$$
\left\|K_{\lambda} V K_{\lambda}: \ell^{2}\left(\Gamma_{b c}\right) \rightarrow \ell^{2}\left(\Gamma_{b c}\right)\right\|<1 .
$$

By proving good estimates from above of the Hilbert-Schmidt norms of the operators $K_{\lambda} V K_{\lambda}$ for $b c=\mathrm{Per}^{ \pm}$or Dir we get the following statements.

Proposition 4. (Theorem 21 in $[\mathbf{1 6}]$ ) For each periodic potential $v \in H_{l o c}^{-1}(\mathbb{R})$, the spectrum of the operator $L_{b c}(v)$ with $b c=P e r^{ \pm}$, Dir is discrete. Moreover, if $b c=P e r^{ \pm}$then, respectively, for each large enough even number $N^{+}>0$ or odd number $N^{-}$, we have

$$
S p\left(L_{P e r^{ \pm}}\right) \subset R_{N^{ \pm}} \cup \bigcup_{n \in N^{ \pm}+2 \mathbb{N}} D_{n},
$$

where the rectangle $R_{N}=\left\{\lambda=x+i y:-N<x<N^{2}+N,|y|<N\right\}$ contains, respectively, $2 N^{+}+1$ or $2 N^{-}$eigenvalues, while each disc $D_{n}=\{\lambda: \mid \lambda-$ $\left.n^{2} \mid<n / 4\right\}$ contains two eigenvalues ( each eigenvalue is counted with its algebraic multiplicity). 
If $b c=$ Dir then, for each large enough $N \in \mathbb{N}$, we have

$$
S p\left(L_{D i r}\right) \subset R_{N} \cup \bigcup_{n=N+1}^{\infty} D_{n}
$$

and

$$
\left.\#\left(S p\left(L_{D i r}\right) \cap R_{N}\right)=N+1, \quad \#\left(S p\left(L_{D i r}\right)\right) \cap D_{n}\right)=1, \quad n>N .
$$

This localization theorem, i.e., Proposition 4, says that for each $n>N^{*}=$ $\max \left\{N^{+}, N^{-}, N\right\}$ the disc $D_{n}$ contains exactly one Dirichlet eigenvalue $\mu_{n}$ and two periodic (if $n$ is even) or antiperiodic (if $n$ is odd) eigenvalues $\lambda_{n}^{+}$and $\lambda_{n}^{-}$, counted with their algebraic multiplicity, where either $\operatorname{Re} \lambda_{n}^{+}>\operatorname{Re} \lambda_{n}^{-}$, or $\operatorname{Re} \lambda_{n}^{+}=\operatorname{Re} \lambda_{n}^{-}$ and $\operatorname{Im} \lambda_{n}^{+} \geq \operatorname{Im} \lambda_{n}^{-}$.

After this observation we define spectral gaps

$$
\gamma_{n}=\left|\lambda_{n}^{+}-\lambda_{n}^{-}\right|, \quad n \geq N^{*}
$$

and deviations

$$
\delta_{n}=\left|\gamma_{n}-\left(\lambda_{n}^{+}+\lambda_{n}^{-}\right) / 2\right|, \quad n \geq N^{*} .
$$

We characterize "the rate of decay" of these sequences by saying that they are elements of an appropriate weight sequence space

$$
\ell^{2}(\Omega)=\left\{\left(x_{k}\right)_{k \in \mathbb{N}}: \sum\left|x_{k}\right|^{2}(\Omega(k))^{2}<\infty\right\} .
$$

The condition (1.10) means that $\ell^{2}(\Omega) \subset \ell^{2}(\{n\})$. Now, with the restriction (1.10) - which is weaker than (1.9) - we permit the weights to decrease to zero. For example, the weights $\Omega_{\beta}(n)=n^{-\beta}, \beta \in(0,1]$, satisfy (1.10). Moreover, if $\beta>1 / 2$, then the sequence $x_{k}=k^{\alpha}, 0<\alpha<\beta-1 / 2$ goes to $\infty$ as $k \rightarrow \infty$ but $\left(x_{k}\right) \in \ell^{2}\left(\Omega_{\beta}\right)$.

By the same token, the "smoothness" of a potential $v$ with Fourier coefficients $V(2 k)$ given by (2.4) is characterized by saying that $v$ belongs to an appropriate weight space $H(\Omega)$, i.e.,

$$
\sum|V(2 k)|^{2}(\Omega(k))^{2}<\infty .
$$

The inclusion $H(\Omega) \subset L^{2}$ is equivalent to (1.9) while (1.10) is equivalent to the inclusion $H(\Omega) \subset H^{-1}$.

Let us recall that a sequence of positive numbers, or a weight $A=(A(n))_{n \in \mathbb{Z}}$, is called sub-multiplicative if

$$
A(m+n) \leq A(m) A(n), \quad m, n \in \mathbb{Z} .
$$

In this paper we often consider weights of the form

$$
a(n)=\frac{A(n)}{n} \quad \text { for } n \neq 0, \quad a(0)=1,
$$

where $A(n)$ is sub-multiplicative and even.

Now we are ready to formulate the main result of the present paper (this is Theorem 28 in Section 7).

Main Theorem. Let $L=L^{0}+v(x)$ be the Hill-Schrödinger operator with a $\pi$-periodic potential $v \in H_{\text {loc }}^{-1}(\mathbb{R})$.

Then, for large enough $n>N(v)$ the operator $L$ has, in a disc of center $n^{2}$ and radius $r_{n}=n / 4$, exactly two (counted with their algebraic multiplicity) periodic 
(for even $n$ ), or antiperiodic (for odd $n$ ) eigenvalues $\lambda_{n}^{+}$and $\lambda_{n}^{-}$, and one Dirichlet eigenvalue $\mu_{n}$.

Let

$$
\Delta_{n}=\left|\lambda_{n}^{+}-\lambda_{n}^{-}\right|+\left|\frac{\lambda_{n}^{+}+\lambda_{n}^{-}}{2}-\mu_{n}\right|, \quad n>N(v),
$$

and let $\Omega=(\Omega(m))_{m \in \mathbb{Z}}, \Omega(m)=\omega(m) / m, m \neq 0$, where $\omega$ is a sub-multiplicative weight. Then we have

$$
v \in H(\Omega) \Rightarrow\left(\Delta_{n}\right) \in \ell^{2}(\Omega) .
$$

Conversely, in the above notations, if $\omega=(\omega(n))_{n \in \mathbb{Z}}$ is a sub-multiplicative weight such that

$$
\frac{\log \omega(n)}{n} \searrow 0
$$

then

$$
\left(\Delta_{n}\right) \in \ell^{2}(\Omega) \Rightarrow v \in H(\Omega)
$$

If $\omega$ is of exponential type, i.e., $\lim _{n \rightarrow \infty} \frac{\log \omega(n)}{n}>0$, then

$$
\left(\Delta_{n}\right) \in \ell^{2}(\Omega) \Rightarrow \exists \varepsilon>0: v \in H\left(e^{\varepsilon|n|}\right) .
$$

Important tool in the proof of this theorem is the following statement. Let $E_{n}$ be the Riesz invariant subspace corresponding to the (periodic for even $n$, or antiperiodic for odd $n$ ) eigenvalues of $L_{P e r^{ \pm}}$lying in the $\operatorname{disc}\left\{z:\left|z-n^{2}\right|<n\right\}$, and let $P_{n}$ be the corresponding Riesz projection, i.e.,

$$
P_{n}=\frac{1}{2 \pi i} \int_{C_{n}}(\lambda-L)^{-1} d \lambda, \quad C_{n}:=\left\{\lambda:\left|\lambda-n^{2}\right|=n\right\} .
$$

We denote by $P_{n}^{0}$ the Riesz projection that corresponds to the free operator.

In the above notations and under the assumptions of Proposition 4

$$
\left\|P_{n}-P_{n}^{0}\right\|_{L^{2} \rightarrow L^{\infty}} \rightarrow 0 \text { as } n \rightarrow \infty \text {. }
$$

This statement and its stronger version are proven in Section 9, Appendix; see Proposition 44 and Theorem 45 there.

\section{Basic equation}

By the localization statement given in Proposition 4, the spectrum of the operator $L_{P e r^{ \pm}}$is discrete, and there exists $N_{*}$ such that for each $n \geq N_{*}$ the disc $D_{n}=\left\{\lambda:\left|\lambda-n^{2}\right|<n / 4\right\}$ contains exactly two eigenvalues (counted with their algebraic multiplicity) of $L_{P e r^{+}}$(for $n$ even), or $L_{P e r^{-}}$(for $n$ odd).

For each $n \in \mathbb{N}$, let

$$
E^{0}=E_{n}^{0}=\operatorname{Span}\left\{e_{-n}=e^{-i n x}, e_{n}=e^{i n x}\right\}
$$

be the eigenspace of the free operator $L^{0}=-d^{2} / d x^{2}$ corresponding to its eigenvalue $n^{2}$ (subject to periodic boundary conditions for even $n$, and antiperiodic boundary conditions for odd $n$ ). We denote by $P^{0}=P_{n}^{0}$ the orthogonal projection on $E^{0}$, and set $Q^{0}=Q_{n}^{0}=1-P_{n}^{0}$. Notice that

$$
D_{n} \cap S p\left(Q^{0} L^{0} Q^{0}\right)=\emptyset \text {. }
$$


Consider the operator $\tilde{K}$ given by its matrix representation

$$
\tilde{K}_{j k}= \begin{cases}\frac{1}{\left(\lambda-k^{2}\right)^{1 / 2}} \delta_{j k} & \text { for } \quad k \neq \pm n \\ 0 & \text { for } \quad k= \pm n\end{cases}
$$

where $z^{1 / 2}:=\sqrt{r} e^{i \varphi / 2}$ if $z=r e^{i \varphi},-\pi \leq \varphi<\pi$, and $j, k \in n+2 \mathbb{Z}$. One can easily see that $\tilde{K}$ acts from $L^{2}([0, \pi])$ into $H^{1}$, and from $H^{-1}$ into $L^{2}([0, \pi])$.

We consider also the operator

$$
T=T(n ; \lambda)=\tilde{K} V \tilde{K}
$$

By the diagram

$$
L^{2}([0, \pi]) \stackrel{\tilde{K}}{\rightarrow} H^{1} \stackrel{V}{\rightarrow} H^{-1} \stackrel{\tilde{K}}{\rightarrow} L^{2}([0, \pi])
$$

the operator $T$ acts in $L^{2}([0, \pi])$. In view of $(2.4)$, (3.2) and (3.3), the matrix representation of $T$ is

$$
T_{j k}= \begin{cases}\frac{V(j-k)}{\left(\lambda-j^{2}\right)^{1 / 2}\left(\lambda-k^{2}\right)^{1 / 2}}=\frac{i(j-k) q(j-k)}{\left(\lambda-j^{2}\right)^{1 / 2}\left(\lambda-k^{2}\right)^{1 / 2}} & \text { if } j, k \neq \pm n \\ 0 & \text { otherwise }\end{cases}
$$

Let us set

$$
H_{n}=\left\{z:(n-1)^{2} \leq \operatorname{Re} z \leq(n+1)^{2}\right\}
$$

and

$$
\mathcal{E}_{m}(q)=\left(\sum_{|k| \geq m}|q(m)|^{2}\right)^{1 / 2}
$$

LEMMA 5. In the above notations, we have

$$
\|T\|_{H S} \leq C\left(\mathcal{E}_{\sqrt{n}}(q)+\|q\| / \sqrt{n}\right), \quad \lambda \in H_{n},
$$

where $C$ is an absolute constant.

ProOF. In view of (3.4),

$$
\|T\|_{H S}^{2}=\sum_{j, k \neq \pm n} \frac{(j-k)^{2}|q(j-k)|^{2}}{\left|\lambda-j^{2}\right|\left|\lambda-k^{2}\right|}
$$

so we have to estimate from above the sum in (3.8) with $\lambda \in H_{n}$.

The operator $\tilde{K}$ is a modification of the operator $K$ defined by

$$
K_{j k}=\frac{1}{\left(\lambda-k^{2}\right)^{1 / 2}} \delta_{j k}, \quad j, k \in n+2 \mathbb{Z}, \lambda \in H_{n} \backslash\left\{n^{2}\right\} .
$$

Moreover, for $\lambda \neq n^{2}$, we have $\tilde{K}=Q^{0} K Q^{0}$.

Therefore (compare (3.8) with Formula (128) in [16]), by repeating the proof of Lemma 19 in [16]) with a few simple changes there one can easily see that (3.7) holds.

LEMMA 6. In the above notations, if $n \in \mathbb{N}$ is large enough and $\lambda \in D_{n}$, then $\lambda$ is an eigenvalue of the operator $L=L^{0}+V$ (considered with periodic boundary conditions if $n$ is even, or antiperiodic boundary conditions if $n$ is odd) if and only if $\lambda$ is an eigenvalue of the operator $P^{0} L^{0} P^{0}+S$, where

$$
S=S(\lambda ; n)=P^{0} V P^{0}+P^{0} V \tilde{K}(1-T)^{-1} \tilde{K} V P^{0}: E_{n}^{0} \rightarrow E_{n}^{0} .
$$


Moreover,

$$
\begin{gathered}
L f=\lambda f, \quad f \neq 0 \Rightarrow\left(L^{0}+S\right) f_{1}=\lambda f_{1}, \quad f_{1}=P^{0} f \neq 0 \\
\left(L^{0}+S\right) f_{1}=\lambda f_{1}, \quad f_{1} \in E^{0} \Rightarrow L f=\lambda f, \quad f=f_{1}+\tilde{K}(1-T)^{-1} \tilde{K} V f_{1} .
\end{gathered}
$$

Proof. The equation

$$
\left(\lambda-L^{0}-V\right) f=g
$$

is equivalent to the system of two equations

$$
\begin{aligned}
& P^{0}\left(\lambda-L^{0}-V\right)\left(f_{1}+f_{2}\right)=g_{1}, \\
& Q^{0}\left(\lambda-L^{0}-V\right)\left(f_{1}+f_{2}\right)=g_{2},
\end{aligned}
$$

where $f_{1}=P^{0} f, f_{2}=Q^{0} f, g_{1}=P^{0} g, g_{2}=Q^{0} g$.

Since the operator $L^{0}$ is self-adjoint, the range $Q^{0}(H)$ of the projection $Q^{0}$ is an invariant subspace of $L^{0}$ also. Therefore,

$$
P^{0} Q^{0}=Q^{0} P^{0}=0, \quad P^{0} L^{0} Q^{0}=Q^{0} L^{0} P^{0}=0,
$$

so Equations (3.13) and (3.14) may be rewritten as

$$
\begin{aligned}
& \left(\lambda-L^{0}\right) f_{1}-P^{0} V f_{1}-P^{0} V f_{2}=g_{1}, \\
& \left(\lambda-L^{0}\right) f_{2}-Q^{0} V f_{1}-Q^{0} V f_{2}=g_{2} .
\end{aligned}
$$

Since $f_{2}$ belongs to the range of $Q^{0}$, it can be written in the form

$$
f_{2}=\tilde{K} \tilde{f}_{2} .
$$

Next we substitute this expression for $f_{2}$ into (3.16), and after that act from the left on the equation by $\tilde{K}$. As a result we get

$$
\tilde{K}\left(\lambda-L^{0}\right) \tilde{K} \tilde{f}_{2}-\tilde{K} Q^{0} V f_{1}-\tilde{K} V \tilde{K} \tilde{f}_{2}=\tilde{K} g_{2} .
$$

By the definition of $\tilde{K}$, we have the identity

$$
\tilde{K}\left(\lambda-L^{0}\right) \tilde{K} \tilde{f}_{2}=\tilde{f}_{2} \text {. }
$$

Therefore, in view of (3.3), the latter equation can be written in the form

$$
(1-T) \tilde{f}_{2}=\tilde{K} V f_{1}+\tilde{K} g_{2} .
$$

By Lemma 5 the operator $1-T$ is invertible for large enough $n$. Thus, (3.17) and (3.18) imply, for large enough $n$,

$$
f_{2}=\tilde{K}(1-T)^{-1} \tilde{K} V f_{1}+\tilde{K}(1-T)^{-1} \tilde{K} g_{2}
$$

By inserting this expression for $f_{2}$ into (3.15) we get

$$
\left(\lambda-P^{0} L^{0} P^{0}-S\right) f_{1}=g_{1}+P^{0} V \tilde{K}(1-T)^{-1} \tilde{K} g_{2},
$$

where the operator $S$ is given by (3.9).

If $\lambda$ is an eigenvalue of $L$ and $f \neq 0$ is a corresponding eigenvector, then (3.12) holds with $g=0$, so $g_{1}=0, g_{2}=0$, and (3.20) implies (3.10), i.e., $\lambda$ is an eigenvalue of $P^{0} L^{0} P^{0}+S$ and $f_{1}=P^{0} f$ is a corresponding eigenvector. Then we have $f_{1} \neq 0$; otherwise (3.19) yields $f_{2}=0$, so $f=f_{1}+f_{2}=0$ which is a contradiction.

Conversely, let $\lambda$ be an eigenvalue of $P^{0} L^{0} P^{0}+S$, and let $f_{1}$ be a corresponding eigenvector. We set

$$
f_{2}=\tilde{K}(1-T)^{-1} \tilde{K} V f_{1}, \quad f=f_{1}+f_{2}
$$


and show that $L f=\lambda f$ by checking that (3.15) and (3.16) hold with $g_{1}=0$ and $g_{2}=0$. Then $(3.15)$ coincides with $\left(P^{0} L^{0} P^{0}+S\right) f_{1}=0$, so it holds. On the other hand one can easily verify (3.16) by using that

$$
(1-T)^{-1}=1+T(1-T)^{-1}, \quad T=\tilde{K} V \tilde{K}, \quad\left(\lambda-L^{0}\right) \tilde{K}^{2}=Q^{0} .
$$

This completes the proof.

Notice that (3.10) and (3.11) imply the following.

REMARK 7. Under the assumptions and notations of Lemma 6, for large enough $n$, the operator $L$ has an eigenvalue $\lambda \in D_{n}$ of geometric multiplicity 2 if and only if $\lambda$ is an eigenvalue of $P^{0} L^{0} P^{0}+S(\lambda, n)$ of geometric multiplicity 2.

Of course, in the proof of Lemma 6 it was enough to consider the equation (3.12) for $g=0$ (and, respectively, to set $g_{1}=0, g_{2}=0$ in the following equations). But we consider the case of arbitrary $g$ in order to explain the following remark that will be used later.

REMARK 8. Under the assumptions and notations of Lemma 6 and its proof, Equation (3.12), i.e., the system (3.13) and (3.14), implies Equation (3.20).

Let $\left(\begin{array}{ll}S^{11} & S^{12} \\ S^{21} & S^{22}\end{array}\right)$ be the matrix representation of the two-dimensional operator $S$ with respect to the basis $e_{n}^{1}:=e_{-n}, e_{n}^{2}:=e_{n}$; then

$$
S^{i j}=\left\langle S e_{n}^{j}, e_{n}^{i}\right\rangle, \quad i, j \in\{1,2\} .
$$

Consider the eigenvalue equation for the operator $S$ :

$$
\operatorname{det}\left|\begin{array}{cc}
S^{11}-z & S^{12} \\
S^{21} & S^{22}-z
\end{array}\right|=0 .
$$

This is the basic equation that we use to estimate spectral gaps.

$A$ number $\lambda=n^{2}+z \in D_{n}$ is a periodic or anti-periodic eigenvalue of $L^{0}+V$ if and only if $z$ is a solution of (3.22).

Next we give explicit formulas for the matrix elements $S^{i j}$. By Lemma 5, we have $\left\|T_{n}\right\| \leq 1 / 2$ for $n \geq n_{0}(v)$, so $\left(1-T_{n}\right)^{-1}=\sum_{k=1}^{\infty} T_{n}^{k-1}$, and therefore,

$$
S=P_{n}^{0} V P_{n}^{0}+\sum_{k=1}^{\infty} P_{n}^{0} V \tilde{K} T_{n}^{k-1} \tilde{K} V P_{n}^{0} .
$$

By (3.9) and (3.21),

$$
S^{i j}=\sum_{k=0}^{\infty} S_{k}^{i j}, \quad \text { where } \quad S_{0}^{i j}=\left\langle P^{0} V P^{0} e_{n}^{j}, e_{n}^{i}\right\rangle
$$

and

$$
S_{k}^{i j}=\left\langle V \tilde{K} T_{n}^{k-1} \tilde{K} V e_{n}^{j}, e_{n}^{i}\right\rangle, \quad k=1,2, \ldots
$$

By (3.3), it follows that

$$
V \tilde{K} T_{n}^{k-1} \tilde{K} V=\left(V \tilde{K}^{2}\right)^{k} V .
$$

Therefore, in view of (3.23), (3.24) and (3.2), we have

$$
S_{0}^{11}=\left\langle V e_{n}^{1}, e_{n}^{1}\right\rangle=V(0)=0, \quad S_{0}^{22}=\left\langle V e_{n}^{2}, e_{n}^{2}\right\rangle=V(0)=0,
$$


and for each $k=1,2, \ldots$,

$$
\begin{aligned}
S_{k}^{11} & =\sum_{j_{1}, \ldots, j_{k} \neq \pm n} \frac{V\left(-n-j_{1}\right) V\left(j_{1}-j_{2}\right) \cdots V\left(j_{k-1}-j_{k}\right) V\left(j_{k}+n\right)}{\left(n^{2}-j_{1}^{2}+z\right) \cdots\left(n^{2}-j_{k}^{2}+z\right)}, \\
S_{k}^{22} & =\sum_{j_{1}, \ldots, j_{k} \neq \pm n} \frac{V\left(n-j_{1}\right) V\left(j_{1}-j_{2}\right) \cdots V\left(j_{k-1}-j_{k}\right) V\left(j_{k}-n\right)}{\left(n^{2}-j_{1}^{2}+z\right) \cdots\left(n^{2}-j_{k}^{2}+z\right)}
\end{aligned}
$$

In the same way, we obtain

$$
S_{0}^{12}=\left\langle V e_{n}^{2}, e_{n}^{1}\right\rangle=V(-2 n), \quad S_{0}^{21}=\left\langle V e_{n}^{1}, e_{n}^{2}\right\rangle=V(2 n),
$$

and, for $k=1,2, \ldots$,

$$
\begin{aligned}
S_{k}^{12} & =\sum_{j_{1}, \ldots, j_{k} \neq \pm n} \frac{V\left(-n-j_{1}\right) V\left(j_{1}-j_{2}\right) \cdots V\left(j_{k-1}-j_{k}\right) V\left(j_{k}-n\right)}{\left(n^{2}-j_{1}^{2}+z\right) \cdots\left(n^{2}-j_{k}^{2}+z\right)} \\
S_{k}^{21} & =\sum_{j_{1}, \ldots, j_{k} \neq \pm n} \frac{V\left(n-j_{1}\right) V\left(j_{1}-j_{2}\right) \cdots V\left(j_{k-1}-j_{k}\right) V\left(j_{k}+n\right)}{\left(n^{2}-j_{1}^{2}+z\right) \cdots\left(n^{2}-j_{k}^{2}+z\right)}
\end{aligned}
$$

The operator $S$ depends on $v, n \in \mathbb{N}$ and $\lambda$ (or, $z=\lambda-n^{2}$ ). Of course, the same is true for its matrix, i.e.,

$$
S^{i j}=S^{i j}(v ; n, z), \quad S_{k}^{i j}=S_{k}^{i j}(v ; n, z) .
$$

Lemma 9. (a) For any (complex-valued) potential $v$

$$
S^{11}(v ; n, z)=S^{22}(v ; n, z) .
$$

(b) If $v$ is a real-valued potential, then

$$
S^{12}(v ; n, z)=\overline{S^{21}(v ; n, \bar{z})} .
$$

Proof. (a) By (3.26) and (3.27), the change of indices

$$
i_{s}=-j_{k+1-s}, \quad s=1, \ldots, k,
$$

proves that $S_{k}^{11}(v ;-n, z)=S_{k}^{22}(v ; n, z)$ for $k=1,2, \ldots$ Thus, in view of (3.23), (3.31) holds.

(b) If $v$ is real-valued, we have for its Fourier coefficients $V(-m)=\overline{V(m)}$. By (3.28),

$$
S_{0}^{12}(v ;-n, z)=V(-2 n)=\overline{V(2 n)}=\overline{S_{0}^{21}(v ; n, \bar{z})} .
$$

By (3.29) and (3.30), for each $k=1,2, \ldots$, the change of indices

$$
i_{s}=j_{k+1-s}, \quad s=1, \ldots, k,
$$

explains that $S_{k}^{12}(-n, z)=\overline{S_{k}^{21}(n, \bar{z})}$. Thus, in view of (3.23), (3.32) holds. Lemma 9 is proved.

We set, for $n>n_{0}(v)$,

$$
\alpha_{n}(v ; z)=S^{11}(v ; n, z), \quad \beta_{n}^{+}(v ; z)=S^{21}(v ; n, z), \quad \beta_{n}^{-}(v ; z)=S^{12}(v ; n, z) .
$$


4. Estimates of $\alpha(v ; n, z)$ and $\beta^{ \pm}(v ; n, z)$

Let $\Omega=(\Omega(m))$ be a weight of the form

$$
\Omega(m)=\omega(m) / m \text { for } m \neq 0 \quad \Omega(0)=1,
$$

where $\omega$ is a submultiplicative weight. Our main goal in this section is to estimate the $\ell^{2}(\Omega)$-norm of the sequence $\left(\beta^{ \pm}(v ; n, z)\right)_{n>N_{0}}$ from (3.33) under the assumption $v \in H(\Omega)$.

Lemma 10. If $r \in \ell^{2}(\mathbb{Z})$, then

$$
\sum_{i \neq-n}\left|\frac{n-i}{n+i}\right|^{2}|r(n-i)|^{2} \leq 8 n^{2}\left(\mathcal{E}_{n}(r)+\frac{\|r\|}{n}\right)^{2}
$$

Proof. Since $|n-i| /|n+i| \leq 1$ for $i \geq 0$, and

$$
\left(\frac{n-i}{n+i}\right)^{2}=\left(\frac{2 n}{n+i}-1\right)^{2} \leq \frac{8 n^{2}}{(n+i)^{2}}+2 \text { for } i<0,
$$

the sum in (4.2) does not exceed

$$
\sum_{i<0, i \neq-n} \frac{8 n^{2}}{(n+i)^{2}}|r(n-i)|^{2}+\sum_{i} 2|r(n-i)|^{2} \leq 8 n^{2}\left(\mathcal{E}_{n}(r)\right)^{2}+2\|r\|^{2},
$$

which proves (4.2).

Lemma 11. If $r \in \ell^{2}(\mathbb{Z})$ and $H(n)=\left(H_{i j}(n)\right), n \in \mathbb{N}, n>N$, are HilbertSchmidt operators such that $H_{*}:=\sup _{n>N}\|H(n)\|_{H S}<\infty$, then

$$
\sum_{i, j \neq \pm n}\left|\frac{n-i}{n+i}\right|^{1 / 2}\left|\frac{n+j}{n-j}\right|^{1 / 2}\left|r(n-i)\|r(n+j)\| H_{i j}(n)\right| \leq C n\|r\|\left(\mathcal{E}_{n}(r)+\frac{\|r\|}{n}\right) H_{*},
$$

where $C$ is an absolute constant.

Proof. By the Cauchy inequality, for each $n \in \mathbb{N}$ with $n>N$, we have

$$
\left(\sum_{i, j \neq \pm n}\left|\frac{n-i}{n+i}\right|^{1 / 2}\left|\frac{n+j}{n-j}\right|^{1 / 2}|r(n-i)||r(n+j)|\left|H_{i j}(n)\right|\right)^{2} \leq \sigma(n)\|H(n)\|_{H S}^{2},
$$

where

$$
\sigma(n)=\sum_{i, j \neq \pm n}\left|\frac{n-i}{n+i}\right|\left|\frac{n+j}{n-j}\right||r(n-i)|^{2}|r(n+j)|^{2} .
$$

The index change $j=-k$ yields

$$
\sigma(n)=\left(\sum_{i \neq \pm n}\left|\frac{n-i}{n+i}\right||r(n-i)|^{2}\right)^{2}
$$

Now the Cauchy inequality and (4.2) imply

$$
\sigma(n) \leq\|r\|^{2} \sum_{i \neq \pm n}\left|\frac{n-i}{n+i}\right|^{2}|r(n-i)|^{2} \leq C n^{2}\|r\|^{2}\left(\mathcal{E}_{n}(r)+\frac{\|r\|}{n}\right)^{2} .
$$


By (4.5) and (4.6), the latter estimate proves (4.4).

LEMMA 12. If $r \in \ell^{2}(\mathbb{Z})$ and $H(n)=\left(H_{i j}(n)\right), n \in \mathbb{N}, n>N$, are HilbertSchmidt operators such that $H_{*}:=\sup _{n>N}\|H(n)\|_{H S}<\infty$, then

$$
\begin{gathered}
\sum_{n>N} \frac{1}{n^{2}} \sum_{i, j \neq \pm n}\left(\left|\frac{n-i}{n+i}\right|^{1 / 2}\left|\frac{n+j}{n-j}\right|^{1 / 2}\left|r(n-i)\|r(n+j)\| H_{i j}(n)\right|\right)^{2} \\
\leq C H_{*}^{2}\|r\|^{2}\left(\left(\mathcal{E}_{N}(r)\right)^{2}+\frac{\|r\|^{2}}{N}\right)
\end{gathered}
$$

where $C$ is an absolute constant.

Proof. Let $\Sigma$ denote the sum on the left of (4.7). By (4.5) we have

$$
\Sigma \leq H_{*}^{2} \sum_{n>N} \frac{1}{n^{2}} \sigma(n)
$$

where $\sigma(n)$ is given by (4.6).

Changing the summation index by $k=-j$ we get

$$
\sigma(n)=\left(\sum_{i \neq \pm n}\left|\frac{n-i}{n+i}\right||r(n-i)|^{2}\right)^{2} \leq 2 \sigma_{1}(n)+2 \sigma_{2}(n)
$$

where

$$
\sigma_{1}(n)=\left(\sum_{i<0, i \neq-n}\left|\frac{n-i}{n+i}\right||r(n-i)|^{2}\right)^{2}, \quad \sigma_{2}(n)=\left(\sum_{i \geq 0} \ldots\right)^{2} .
$$

By the Cauchy inequality and (4.3), we have

$$
\sum_{n>N} \frac{1}{n^{2}} \sigma_{1}(n) \leq\|r\|^{2}\left(\sum_{n>N} \sum_{i<0, i \neq-n} \frac{8}{(n+i)^{2}}|r(n-i)|^{2}+\sum_{n>N} \frac{2}{n^{2}}\|r\|^{2}\right) .
$$

Now, with $\nu=n-i$,

$$
\sum_{n>N} \sum_{i<0, i \neq-n} \frac{8}{(n+i)^{2}}|r(n-i)|^{2} \leq \sum_{\nu>N}|r(\nu)|^{2} \sum_{n \neq \nu / 2} \frac{8}{(2 n-\nu)^{2}} \leq \frac{8 \pi^{2}}{3}\left(\mathcal{E}_{N}(r)\right)^{2},
$$

so it follows that

$$
\sum_{n>N} \frac{1}{n^{2}} \sigma_{1}(n) \leq\|r\|^{2}\left(\frac{8 \pi^{2}}{3}\left(\mathcal{E}_{N}(r)\right)^{2}+\frac{2\|r\|^{2}}{N}\right) .
$$

On the other hand, since $|n-i| /|n+i| \leq 1$ for $i \geq 0$, we have $\sigma_{2}(n) \leq\|r\|^{4}$. Thus,

$$
\sum_{n>N} \frac{1}{n^{2}} \sigma_{2}(n) \leq \sum_{n>N} \frac{1}{n^{2}}\|r\|^{4} \leq \frac{1}{N}\|r\|^{4} .
$$

Finally, (4.8)-(4.12) imply (4.7), which completes the proof.

Let us recall that a weight $\omega$ is called slowly increasing, if $\sup _{n} \frac{\omega(2 n)}{\omega(n)}<\infty$. 
Lemma 13. Suppose $\omega=(\omega(m))_{m \in 2 \mathbb{Z}}$ is a slowly increasing weight such that

$$
\omega(m) \leq C_{1}|m|^{1 / 4}, \quad m \in 2 \mathbb{Z},
$$

$r=(r(m))_{m \in 2 \mathbb{Z}} \in \ell^{2}(\omega, 2 \mathbb{Z})$, and $H(n)=\left(H_{i j}(n)\right)_{i, j \in n+2 \mathbb{Z}}, n \geq n_{0}$, are Hilbert-

Schmidt operators such that $H_{*}=\sup _{n \geq n_{0}}\|H(n)\|_{H S}<\infty$. Then, for $N>n_{0}$,

$$
\begin{gathered}
\sum_{n>N} \frac{(\omega(2 n))^{2}}{n^{2}} \sum_{i, j \neq \pm n}\left(\left|\frac{n-i}{n+i}\right|^{1 / 2}\left|\frac{n+j}{n-j}\right|^{1 / 2}|r(n-i) \| r(n+j)|\left|H_{i j}(n)\right|\right)^{2} \\
\leq C H_{*}^{2}\|r\|_{\omega}^{4}\left(\frac{1}{(\omega(N))^{2}}+\frac{1}{\sqrt{N}}\right)
\end{gathered}
$$

where $C=C(\omega)$.

Proof. Let $\Sigma$ denote the sum on the left of (4.14). By (4.5) we have

$$
\Sigma \leq H_{*}^{2} \sum_{n>N} \frac{(\omega(2 n))^{2}}{n^{2}} \sigma(n),
$$

where $\sigma(n)$ is given by (4.6), so (4.9) holds, i.e.,

$$
\sigma(n) \leq 2 \sigma_{1}(n)+2 \sigma_{2}(n)
$$

with $\sigma_{1}(n)$ and $\sigma_{2}(n)$ defined by (4.10).

Consider the sequence $\bar{r}=(\bar{r}(m))_{m \in 2 \mathbb{Z}}$ defined by

$$
\bar{r}(m)=\omega(m) r(m), \quad m \in 2 \mathbb{Z} .
$$

Then $\bar{r} \in \ell^{2}$ and $\|\bar{r}\|=\|r\|_{\omega}$.

Taking into account that

$$
\bar{r}(n-i)=\omega(n-i) r(n-i) \geq \omega(n) r(n-i) \quad \text { if } i<0,
$$

we estimate from above:

$$
\begin{aligned}
& \sigma_{1}(n) \leq \frac{1}{(\omega(n))^{4}}\left(\sum_{i<0, i \neq-n}\left|\frac{n-i}{n+i}\right||\bar{r}(n-i)|^{2}\right)^{2} \\
& \leq \frac{1}{(\omega(n))^{4}}\left(\sum_{i<0, i \neq-n}\left|\frac{n-i}{n+i}\right|^{2}|\bar{r}(n-i)|^{2}\right) \cdot\|\bar{r}\|^{2}
\end{aligned}
$$

(by the Cauchy inequality).

Since $\omega$ is a slowly increasing weight, there is a constant $C_{0}>0$ such that

$$
\omega(2 n) \leq C_{0} \omega(n) \quad \forall n \in \mathbb{N} .
$$

Therefore, in view of (4.3), we have

$$
\begin{aligned}
& \sum_{n>N} \frac{(\omega(2 n))^{2}}{n^{2}} \sigma_{1}(n) \leq\|\bar{r}\|^{2} \sum_{n>N} \frac{C_{0}^{2}}{(\omega(n))^{2}} \sum_{i<0, i \neq-n}\left(\frac{8}{(n+i)^{2}}+\frac{2}{n^{2}}\right)|\bar{r}(n-i)|^{2} \\
& \leq \frac{C_{0}^{2}\|\bar{r}\|^{2}}{(\omega(N))^{2}}\left(\sum_{n>N} \sum_{i<0, i \neq-n} \frac{8}{(n+i)^{2}}|\bar{r}(n-i)|^{2}+\sum_{n>N} \frac{2}{n^{2}} \sum_{i}|\bar{r}(n-i)|^{2}\right) .
\end{aligned}
$$


Since we have, with $\nu=n-i$,

$$
\sum_{n>N} \sum_{i<0, i \neq-n} \frac{8}{(n+i)^{2}}|\bar{r}(n-i)|^{2} \leq \sum_{\nu>N}|\bar{r}(\nu)|^{2} \sum_{n \neq \nu / 2} \frac{8}{(2 n-\nu)^{2}} \leq \frac{8 \pi^{2}}{3}\left(\mathcal{E}_{N}(\bar{r})\right)^{2},
$$

it follows that

$$
\sum_{n>N} \frac{(\omega(2 n))^{2}}{n^{2}} \sigma_{1}(n) \leq \frac{C_{0}^{2}\|\bar{r}\|^{4}}{(\omega(N))^{2}}\left(\frac{8 \pi^{2}}{3}+2\right) .
$$

On the other hand, since $|n-i| /|n+i| \leq 1$ for $i \geq 0$, we have

$$
\sigma_{2}(n) \leq\|r\|^{4} \text {. }
$$

Thus, by (4.13),

$$
\sum_{n>N} \frac{(\omega(2 n))^{2}}{n^{2}} \sigma_{2}(n) \leq \sum_{n>N} \frac{C_{1}^{2}(2 n)^{1 / 2}}{n^{2}}\|r\|^{4} \leq 2 \sqrt{2} C_{1}^{2} \frac{\|r\|^{4}}{\sqrt{N}} .
$$

Now (since $\|r\| \leq\|\bar{r}\|$ ) and $\|\bar{r}\|=\|r\|_{\omega}$ ) (4.15)-(4.16) imply (4.14), which completes the proof.

We set, for each sequence $\rho=(\rho(m))_{m \in 2 \mathbb{Z}}$ such that

$$
\begin{gathered}
\rho(0)=0, \quad \sum_{m \neq 0}(\rho(m))^{2} / m^{2}<\infty \\
A_{k}(\rho ; n)=\sum_{j_{1}, \ldots, j_{k} \neq \pm n} \frac{\left|\rho\left(n-j_{1}\right) \rho\left(j_{1}-j_{2}\right) \cdots \rho\left(j_{k}-n\right)\right|}{\left|n^{2}-j_{1}^{2}\right| \cdots\left|n^{2}-j_{k}^{2}\right|}, \quad k, n \in \mathbb{N}, \\
B_{k}(\rho ; \pm n)=\sum_{j_{1}, \ldots, j_{k} \neq \pm n} \frac{\left|\rho\left( \pm n-j_{1}\right) \rho\left(j_{1}-j_{2}\right) \cdots \rho\left(j_{k} \pm n\right)\right|}{\left|n^{2}-j_{1}^{2}\right| \cdots\left|n^{2}-j_{k}^{2}\right|}, \quad k, n \in \mathbb{N},
\end{gathered}
$$

and

$$
A(\rho ; n)=\sum_{k=1}^{\infty} A_{k}(\rho ; n), \quad B(\rho ; \pm n)=\sum_{k=1}^{\infty} B_{k}(\rho ; \pm n) .
$$

By the elementary inequality, for $n \geq 2$,

$$
\frac{1}{\left|n^{2}+z-j^{2}\right|} \leq \frac{2}{\left|n^{2}-j^{2}\right|}, \quad j \in n+2 \mathbb{Z}, \quad j \neq \pm n, \quad|z| \leq n / 2,
$$

(3.26),(3.29), (3.30) and (3.31) imply, for $k \in \mathbb{N},|z| \leq n / 2$, that

$$
\begin{gathered}
\left|S_{k}^{11}(v ; n, z)\right|=\left|S_{k}^{22}(v ; n, z)\right| \leq A_{k}(2 V ; n), \\
\left|S_{k}^{12}(v ; n, z)\right| \leq B_{k}(2 V ;-n), \quad\left|S_{k}^{21}(v ; n, z)\right| \leq B_{k}(2 V ; n),
\end{gathered}
$$

where $V=(V(m))_{m \in 2 \mathbb{Z}}$ is the sequence of the Fourier coefficients of the potential $v(x)=\sum_{m \in 2 \mathbb{Z}} V(m) \exp (i m x)$. Therefore, by (3.23), (3.28), (3.33) and (4.21), we have

(4.24) $\quad\left|\alpha_{n}(v ; z)\right| \leq A(2 V ; n), \quad \mid \beta_{n}^{ \pm}(v ; z)-V( \pm 2 n|\leq B(2 V ; \pm n), \quad| z \mid<n / 2$.

In view of (4.19)-(4.21) we have

$$
\begin{aligned}
& A(\rho, n)=\left\langle\hat{V} \hat{K}^{2} \hat{V} e_{n}, e_{n}\right\rangle+\left\langle\hat{V} \hat{K} \hat{T}(1-\hat{T})^{-1} \hat{K} \hat{V} e_{n}, e_{n}\right\rangle, \\
& B(\rho, \pm n)=\left\langle\hat{V} \hat{K}^{2} \hat{V} e_{\mp n}, e_{ \pm n}\right\rangle+\left\langle\hat{V} \hat{K} \hat{T}(1-\hat{T})^{-1} \hat{K} \hat{V} e_{\mp n}, e_{ \pm n}\right\rangle
\end{aligned}
$$


where $\hat{V}$ and $\hat{K}$ denote, respectively, the operators with matrix representations

$$
\hat{V}_{i j}=|\rho(i-j)|, \quad \hat{K}_{i j}= \begin{cases}\frac{1}{\left|n^{2}-j^{2}\right|^{1 / 2}} \delta_{i j} & \text { if } i, j \neq \pm n, \\ 0 & \text { if } i= \pm n, \text { or } j= \pm n,\end{cases}
$$

and

$$
\hat{T}=\hat{T}_{n}=\hat{K} \hat{V} \hat{K}
$$

The operator $\hat{T}$ is a slight modification of the operator $T$ defined by (3.3). Therefore, one can easily see that we have the same estimate for its Hilbert-Schmidt norm, namely (compare with (3.3)),

$$
\|\hat{T}\|_{H S} \leq C\left(\mathcal{E}_{\sqrt{n}}(r)+\frac{\|r\|}{\sqrt{n}}\right)
$$

where $r=(r(m))$ is an $\ell^{2}$-sequence defined by $\rho$ from (4.18) so that

$$
r(0)=0, \quad r(m)=\rho(m) / m \quad \text { for } m \neq 0,
$$

and $C$ is an absolute constant.

LEMma 14. Under the above assumptions and notations, there is $n_{0}=n_{0}(\rho)$ such that, for $n>n_{0}$, we have

$$
A(\rho ; n) \leq C n\left(\mathcal{E}_{\sqrt{n}}(r)+\frac{\|r\|}{\sqrt{n}}\right)\|r\|, \quad B(\rho ; \pm n) \leq C n\left(\mathcal{E}_{\sqrt{n}}(r)+\frac{\|r\|}{\sqrt{n}}\right)\|r\|,
$$

where $C$ is an absolute constant.

Proof. By (4.25),

$$
A(\rho, n)=\left\langle\hat{V} \hat{K}^{2} \hat{V} e_{n}, e_{n}\right\rangle+\left\langle\hat{V} \hat{K} \hat{T}(1-\hat{T})^{-1} \hat{K} \hat{V} e_{n}, e_{n}\right\rangle=\Sigma_{1}+\Sigma_{2} .
$$

In view of (4.19)-(4.21) and (4.30), we have

$$
\begin{aligned}
& \Sigma_{1} \leq \sum_{j \neq \pm n} \frac{|\rho(n-j) \rho(j-n)|}{\left|n^{2}-j^{2}\right|} \leq \sum_{j \neq \pm n}\left|\frac{n-j}{n+j}\right||r(n-j)||r(j-n)| \\
& \leq\left(\sum_{j \neq \pm n}\left|\frac{n-j}{n+j}\right|^{2}|r(n-j)|^{2}\right)^{1 / 2} \cdot\|r\| \leq C n\left(\mathcal{E}_{\sqrt{n}}(r)+\frac{\|r\|}{\sqrt{n}}\right)\|r\|
\end{aligned}
$$

(by the Cauchy inequality and Lemma 10). In an analogous way we get

$$
\begin{gathered}
\Sigma_{2} \leq \sum_{i, j \neq \pm n} \frac{|\rho(n-i) \rho(j-n)|}{\left|n^{2}-i^{2}\right|^{1 / 2}\left|n^{2}-j^{2}\right|^{1 / 2}} H_{i j}(n) \\
=\sum_{i, j \neq \pm n}\left|\frac{n-i}{n+i}\right|^{1 / 2}\left|\frac{n+j}{n-j}\right|^{1 / 2}|r(n-i)||r(n+j)| H_{i j}(n),
\end{gathered}
$$

where $H(n)=\hat{T}(1-\hat{T})^{-1}$, so $H_{i j} \geq 0$. By $(4.30),\|\hat{T}\| \leq 1 / 2$ for $n>n_{0}$, which implies

$$
\|H(n)\| \leq 2\|\hat{T}\| \leq 1 \quad \text { for } n>n_{0} .
$$

Thus, by Lemma 11, we get

$$
\Sigma_{2} \leq C n\|r\|\left(\mathcal{E}_{n}(r)+\frac{\|r\|}{n}\right)
$$


where $C$ is an absolute constant.

The obtained estimates of $\Sigma_{1}$ and $\Sigma_{2}$ imply the first inequality in (4.31). We omit the proof of the second inequality there (which gives an upper bound for $B(\rho, n))$, because it is practically the same.

Proposition 15. Let $v$ be a $H^{-1}$-potential of the form (1.2) with (1.3), (1.4), and let (2.4) define the sequence of its Fourier coefficients $V=(V(m))_{m \in 2 \mathbb{Z} \text {. }}$

There exists $n_{0}(v)$ such that for $n>n_{0}$ we have

$$
\left|\alpha_{n}(v ; z)\right|,\left|\beta_{n}^{ \pm}(v ; z)-V( \pm 2 n)\right| \leq C n\left(\mathcal{E}_{\sqrt{n}}(q)+\frac{\|q\|}{\sqrt{n}}\right)\|q\| \quad \text { for }|z| \leq n / 2,
$$

and moreover,

$$
\left|\frac{\partial \alpha_{n}(v ; z)}{\partial z}\right|,\left|\frac{\partial \beta_{n}(v ; z)}{\partial z}\right| \leq 2 C\left(\mathcal{E}_{\sqrt{n}}(q)+\frac{\|q\|}{\sqrt{n}}\right)\|q\| \quad \text { for }|z| \leq n / 4
$$

where $C$ is an absolute constant.

Proof. In view of (4.24), (4.32) follows immediately from Lemma 14.

Since $\alpha_{n}$ and $\beta_{n}^{ \pm}$depend analytically on $z$ for $|z| \leq n / 2$, the Cauchy inequality for their derivatives proves (4.33). Proposition 15 is proved.

Lemma 16. Suppose $\omega=(\omega(m))_{m \in 2 \mathbb{Z}}$ is a weight such that

$$
\omega(m)=\omega_{1}(m) / m, \text { for } m \neq 0,
$$

where $\omega_{1}=\left(\omega_{1}(m)\right)_{m \in 2 \mathbb{Z}}$ is a slowly increasing unbounded weight such that

$$
\omega_{1}(m) \leq C_{1}|m|^{1 / 4}, \quad m \in 2 \mathbb{Z} .
$$

If $\rho=(\rho(m))_{m \in 2 \mathbb{Z}} \in \ell^{2}(\omega, 2 \mathbb{Z}), \rho(0)=0$, then there exists $n_{0}\left(\|\rho\|_{\omega}, \omega_{1}\right)$ such that

$$
\sum_{n>N}(\omega(2 n))^{2}|B(\rho ; \pm n)|^{2} \leq C\|\rho\|_{\omega}^{4}\left(\frac{1}{\left(\omega_{1}(N)\right)^{2}}+\frac{1}{\sqrt{N}}\right), \quad N>n_{0},
$$

where $C=C\left(\omega_{1}\right)$.

Proof. By changing the summation indices one can easily see that

$$
B_{k}(\rho ;-n)=B_{k}(\tilde{\rho} ; n),
$$

where the sequence $\tilde{\rho}$ is defined by $\tilde{\rho}(j)=\rho(-j)$, so we have

$$
B(\rho ;-n)=B(\tilde{\rho} ; n) .
$$

Thus, it is enough to consider only the case of positive $n$ in (4.36).

Consider the sequences $r=(r(m))$ and $\bar{r}=(\bar{r}(m))$ defined by

$$
r(0)=0, \quad r(m)=\rho(m) / m \quad \text { for } m \neq 0, \quad \bar{r}(m)=\omega_{1}(m) r(m) .
$$

Then we have

$$
r \in \ell^{2}\left(\omega_{1}, 2 \mathbb{Z}\right), \quad \bar{r} \in \ell^{2}(2 \mathbb{Z}), \quad\|\bar{r}\|=\|r\|_{\omega_{1}}=\|\rho\|_{\omega} .
$$

Since $\omega_{1}$ is a slowly increasing weight, there exists $C_{0} \geq 1$ such that

$$
\omega_{1}(2 m) \leq C_{0} \omega_{1}(m), \quad m \in 2 \mathbb{Z} .
$$

One can easily see that the weight $C_{0} \omega_{1}(m)$ is sub-multiplicative. Therefore,

$$
\omega_{1}(2 n) \leq C_{0} \omega_{1}(n-j) \omega_{1}(n+j), \quad j \in 2 \mathbb{Z} .
$$


By (4.26) we have

$$
B(\rho ; n)=\left\langle\hat{V} \hat{K}^{2} \hat{V} e_{-n}, e_{n}\right\rangle+\left\langle\hat{V} \hat{K} \hat{T}(1-\hat{T})^{-1} \hat{K} \hat{V} e_{-n}, e_{n}\right\rangle=\Sigma_{3}+\Sigma_{4}
$$

By (4.20),(4.21), (4.27) and (4.37)

$$
\Sigma_{3}=\left\langle\hat{V} \hat{K}^{2} \hat{V} e_{-n}, e_{n}\right\rangle=\sum_{j \neq \pm n} \frac{|\rho(n-j)||\rho(j+n)|}{\left|n^{2}-j^{2}\right|} \leq \sum_{j \neq \pm n}|r(n-j)||r(j+n)| .
$$

Therefore, from $(4.37)-(4.39)$ it follows that

$$
\sum_{n>N} \frac{\left(\omega_{1}(2 n)\right)^{2}}{n^{2}}\left|\Sigma_{3}\right|^{2} \leq \sum_{n>N} \frac{1}{n^{2}}\left(\sum_{j \neq \pm n} C_{0}|\bar{r}(n-j) \| \bar{r}(j+n)|\right)^{2} \leq \frac{1}{N} C_{0}^{2}\|\bar{r}\|^{4} .
$$

On the other hand, (4.20),(4.21), (4.28) and (4.37) imply that

$$
\begin{aligned}
\Sigma_{4}= & \left\langle\hat{V} \hat{K} H(n) \hat{K} \hat{V} e_{-n}, e_{n}\right\rangle \leq \sum_{i, j \neq \pm n} \frac{|\rho(n-i)||\rho(j+n)|}{\left|n^{2}-i^{2}\right|^{1 / 2}\left|n^{2}-j^{2}\right|^{1 / 2}} H_{i j}(n) \\
& =\sum_{i, j \neq \pm n}\left|\frac{n-i}{n+i}\right|^{1 / 2}\left|\frac{n+j}{n-j}\right|^{1 / 2}|r(n-i)||r(n+j)| H_{i j}(n),
\end{aligned}
$$

where $H(n)=\hat{T}(1-\hat{T})^{-1}$. By (4.29) and (4.37) we have

$$
\|\hat{T}\|_{H S} \leq C\left(\mathcal{E}_{\sqrt{n}}(r)+\frac{\|r\|}{\sqrt{n}}\right)
$$

which implies, since $\omega_{1}$ is unbounded,

$$
\|\hat{T}\|_{H S} \leq C\left(\frac{\mathcal{E}_{\sqrt{n}}(\bar{r})}{\omega_{1}(\sqrt{n})}+\frac{\|r\|}{\sqrt{n}}\right) \leq C\left(\frac{1}{\omega_{1}(\sqrt{n})}+\frac{1}{\sqrt{n}}\right) \cdot\|\rho\|_{\omega} \leq \frac{1}{2}
$$

for $n>n_{0}\left(\|\rho\|_{\omega}, \omega_{1}\right)$. Therefore, for $n>n_{0}$, it follows that

$$
\|H(n)\|_{H S}=\left\|\hat{T}(1-\hat{T})^{-1}\right\|_{H S} \leq 2\|\hat{T}\|_{H S} \leq 1 .
$$

Hence, by Lemma 13, we get

$$
\sum_{n>N} \frac{\left(\omega_{1}(2 n)\right)^{2}}{n^{2}}\left|\Sigma_{4}\right|^{2} \leq C\|\bar{r}\|^{4}\left(\frac{1}{\left(\omega_{1}(N)\right)^{2}}+\frac{1}{\sqrt{N}}\right)
$$

Now (4.34), (4.41) and (4.44) imply (4.36), may be with another $n_{0}$. This completes the proof.

In Lemma 16 we assume that the weight $\omega_{1}$ is unbounded, and this assumption is used to get (4.43). But if $\omega_{1}$ is bounded, say $\omega_{1}(k) \equiv 1$, (4.42) implies $\|\hat{T}\|_{H S} \leq$ $1 / 2$ for $n>n_{0}(r)$, so we have $\|H(n)\|_{H S} \leq 1$ for $n>n_{0}$.

Hence, by Lemma 12, we get

$$
\sum_{n>N} \frac{1}{n^{2}}\left|\Sigma_{4}\right|^{2} \leq C\|r\|^{2}\left(\left(\mathcal{E}_{N}(r)\right)^{2}+\frac{\|r\|^{2}}{N}\right)
$$

where $C$ is an absolute constant. Since all other estimates in the proof of Lemma 16 hold with $r=\bar{r}$, we get the following statement. 
LEMMA 17. If $r=(r(m))_{m \in 2 \mathbb{Z}} \in \ell^{2}(2 \mathbb{Z})$ and $\rho=(\rho(m)), \rho(m)=m r(m)$, then

$$
\sum_{n>N} \frac{1}{n^{2}}|B(\rho ; \pm n)|^{2} \leq C\|r\|^{2}\left(\left(\mathcal{E}_{N}(r)\right)^{2}+\frac{\|r\|^{2}}{N}\right) \text {, }
$$

where $C$ is an absolute constant.

If $\omega=(\omega(m))_{m \in 2 \mathbb{Z}}$ is a weight, then we set $\omega \rho=(\omega(m) \rho(m))_{m \in 2 \mathbb{Z}}$.

LEMMA 18. In the notations (4.20)-(4.21), if $\omega$ is a sub-multiplicative weight on $2 \mathbb{Z}$, then

$$
B(\rho ; \pm n) \omega(2 n) \leq B(\omega \rho ; \pm n) .
$$

Proof. In view of (4.21), it is enough to show that

$$
B_{k}(\rho ; \pm n) \omega(2 n) \leq B_{k}(\omega \rho ; \pm n), \quad k \in \mathbb{N} .
$$

Since $\omega$ is a sub-multiplicative weight, for each $k$-tuple of indices $j_{1}, \ldots, j_{k}$ we have

$$
\omega(2 n)=\omega(-2 n) \leq \omega\left( \pm n-j_{1}\right) \omega\left(j_{1}-j_{2}\right) \cdots \omega\left(j_{k-1}-j_{k}\right) \omega\left(j_{k} \pm n\right) .
$$

Thus

$$
\begin{gathered}
\rho\left( \pm n-j_{1}\right) \rho\left(j_{1}-j_{2}\right) \cdots \rho\left(j_{k} \pm n\right) \omega(2 n) \leq \\
{\left[\rho\left( \pm n-j_{1}\right) \omega\left( \pm n-j_{1}\right)\right]\left[\rho\left(j_{1}-j_{2}\right) \omega\left(j_{1}-j_{2}\right)\right] \cdots\left[\rho\left(j_{k} \pm n\right) \omega\left(j_{k} \pm n\right)\right],}
\end{gathered}
$$

which implies (4.48). This proves Lemma 18.

Proposition 19. Suppose $\Omega=(\Omega(k))_{k \in \mathbb{Z}}$ is a weight of the form

$$
\Omega(k)=\frac{\Omega_{1}(k) \Omega_{2}(k)}{k}, \quad k \in \mathbb{Z},
$$

where $\Omega_{1}$ is a slowly increasing weight such that

$$
\Omega_{1}(k) \leq C_{1}|k|^{1 / 4},
$$

and $\Omega_{2}$ is a sub-multiplicative weight.

If $v \in H(\Omega)$, then, for large enough $N$, we have

$$
\begin{gathered}
\sum_{n>N}\left(\left|\beta_{n}^{-}(z)-V(-2 n)\right|^{2}+\left|\beta_{n}^{+}(z)-V(2 n)\right|^{2}\right)(\Omega(n))^{2} \\
\leq C\left(\frac{1}{\left(\Omega_{1}(N)\right)^{2}}+\frac{1}{\sqrt{N}}\right)\|v\|_{\Omega}^{4}, \quad|z|<n / 2,
\end{gathered}
$$

where $C=C\left(\Omega_{1}\right) \geq 1$.

Proof. Consider the weight $\omega=(\omega(m))_{m \in 2 \mathbb{Z}}$, where $\omega(m)=\Omega(m / 2)$. Then we have $\omega(m)=2 \omega_{1}(m) \omega_{2}(m) / m$, where $\omega_{1}$ is a slowly increasing weight such that $\omega_{1}(m)=\Omega_{1}(m / 2)$, and $\omega_{2}$ is a sub-multiplicative weight such that $\omega_{2}(m)=$ $\Omega_{2}(m / 2)$.

Consider also the sequence $\rho=(\rho(m))_{m \in 2 \mathbb{Z}}$, defined by

$$
\rho(m)=2 \max (|V(-m)|,|V(m)|) .
$$

Then we have $\rho \in \ell^{2}(\omega)$, and moreover,

$$
\frac{1}{4}\|\rho\|_{\omega} \leq\|v\|_{\Omega} \leq\|\rho\|_{\omega} .
$$


Since $\rho(-m)=\rho(m)$, we have $B(\rho ;-n)=B(\rho ; n)$, and therefore, by (4.24),

$$
\left|\beta_{n}^{-}(z)-V(-2 n)\right|+\left|\beta_{n}^{+}(z)-V(2 n)\right| \leq 2 B(\rho ; n) .
$$

Thus, it is enough to estimate $\sum_{n>N}|B(\rho ; n)|^{2}(\omega(2 n))^{2}$.

By Lemma 18, we have

$$
B(\rho ; n) \omega_{2}(2 n) \leq B\left(\omega_{2} \rho ; n\right)
$$

and therefore,

$$
\sum_{n>N}|B(\rho ; n)|^{2}(\omega(2 n))^{2} \leq \sum_{n>N}\left|B\left(\omega_{2} \rho ; n\right)\right|^{2} \frac{\left(\omega_{1}(2 n)\right)^{2}}{n^{2}} .
$$

In view of (4.36) in Lemma 16 and the identity $\left\|\omega_{2} \rho\right\|_{\omega_{1} / n}=\|\rho\|$, the latter sum does not exceed

$$
C\left(\frac{1}{\left(\Omega_{1}(N)\right)^{2}}+\frac{1}{N}\right)\|\rho\|_{\omega}^{4}
$$

where $C=C\left(\omega_{1}\right)$. In view of (4.52), this completes the proof of Proposition 19 .

\section{Estimates for $\gamma_{n}$.}

In this section we give estimates of $\gamma_{n}$ from above and below in terms of matrix elements (3.21) of operators $S$ in Basic Equation (3.22), i.e., in terms of $\alpha_{n}(v ; z), \beta_{n}^{ \pm}(v ; z)$ defined in $(3.33)$.

The proofs are essentially the same as in the case of $L^{2}$-potentials, provided the necessary a priori estimates of $\alpha_{n}(v ; z), \beta^{ \pm}(v ; z)$ and their derivatives are proved (which is done in Section 4, Proposition 15).

By Proposition 4, if the potential $v \in H_{l o c}^{-1}(\mathbb{R})$ is $\pi$-periodic, then the operator $L_{P e r^{ \pm}}$has exactly two eigenvalues $\lambda_{n}^{-}$and $\lambda_{n}^{+}$in the $\operatorname{disc} D_{n}=\left\{\lambda^{2}+z,|z|<n / 4\right\}$ (counted with their multiplicity, periodic for even $n$, or antiperiodic for odd $n$ ).

By Lemma 6 and Remark 7, the numbers

$$
z_{n}^{-}=\lambda_{n}^{-}-n^{2} \quad \text { and } \quad z_{n}^{+}=\lambda_{n}^{+}-n^{2}
$$

are eigenvalues of the operator $S$ defined in (3.9), and therefore, $z_{n}^{+}$and $z_{n}^{-}$are roots of the basic equation (3.22). Let us rewrite (3.22) in the form

$$
\left(\zeta_{n}(z)\right)^{2}=\beta_{n}^{-}(z) \cdot \beta_{n}^{+}(z)
$$

where

$$
\zeta_{n}(z)=z-\alpha_{n}(v ; z)
$$

By Proposition 15, (4.33),

$$
\sup _{\left[z_{n}^{-}, z_{n}^{+}\right]}\left|\partial \alpha_{n} / \partial z\right| \leq \varepsilon_{n}, \quad \sup _{\left[z_{n}^{-}, z_{n}^{+}\right]}\left|\partial \beta_{n}^{ \pm} / \partial z\right| \leq \varepsilon_{n},
$$

where $\left[z_{n}^{-}, z_{n}^{+}\right]$denotes the segment with end points $z_{n}^{-}$and $z_{n}^{+}$, and

$$
\varepsilon_{n}=2 C\left(\mathcal{E}_{\sqrt{n}}(q)+\frac{\|q\|}{\sqrt{n}}\right)\|q\| \rightarrow 0 \quad \text { as } n \rightarrow \infty .
$$

Therefore, in view of (5.3),

$$
\begin{gathered}
\left|z_{n}^{+}-z_{n}^{-}\right| \leq\left|\zeta_{n}\left(z_{n}^{+}\right)-\zeta_{n}\left(z_{n}^{-}\right)\right|+\left|\alpha\left(z_{n}^{+}\right)-\alpha\left(z_{n}^{-}\right)\right| \\
\leq\left|\zeta_{n}\left(z_{n}^{+}\right)-\zeta_{n}\left(z_{n}^{-}\right)\right|+\varepsilon_{n} \cdot\left|z_{n}^{+}-z_{n}^{-}\right|
\end{gathered}
$$


which yields

$$
\left(1-\varepsilon_{n}\right)\left|z_{n}^{+}-z_{n}^{-}\right| \leq\left|\zeta_{n}\left(z_{n}^{+}\right)-\zeta_{n}\left(z_{n}^{-}\right)\right| .
$$

On the other hand, in view of (5.4), the identity

implies

$$
\zeta_{n}\left(z_{n}^{+}\right)-\zeta_{n}\left(z_{n}^{-}\right)=\int_{z_{n}^{-}}^{z_{n}^{+}}\left(1-\partial \alpha_{n} / \partial z\right) d z
$$

$$
\left|\zeta_{n}\left(z_{n}^{+}\right)-\zeta_{n}\left(z_{n}^{-}\right)\right| \leq\left(1+\varepsilon_{n}\right)\left|z_{n}^{+}-z_{n}^{-}\right| .
$$

Thus, we have the following two-side estimate:

$$
\left(1-\varepsilon_{n}\right)\left|z_{n}^{+}-z_{n}^{-}\right| \leq\left|\zeta_{n}\left(z_{n}^{+}\right)-\zeta_{n}\left(z_{n}^{-}\right)\right| \leq\left(1+\varepsilon_{n}\right)\left|z_{n}^{+}-z_{n}^{-}\right| .
$$

LEMMA 20. In the above notations, for large enough $n$, we have

$$
\gamma_{n}=\left|\lambda_{n}^{+}-\lambda_{n}^{-}\right| \leq\left(1+\eta_{n}\right)\left(\left|\beta_{n}^{-}\left(z_{n}^{*}\right)\right|+\left|\beta_{n}^{+}\left(z_{n}^{*}\right)\right|\right), \quad z_{n}^{*}=\frac{\lambda_{n}^{+}+\lambda_{n}^{-}}{2}-n^{2},
$$

with $\eta_{n} \rightarrow 0$ as $n \rightarrow \infty$.

Proof. By Equation (5.2),

$$
\left|\zeta_{n}(z)\right| \leq \frac{1}{2}\left(\left|\beta_{n}^{-}(z)\right|+\left|\beta_{n}^{+}(z)\right|\right) \quad \text { for } \quad z=z_{n}^{ \pm} .
$$

Thus, by (5.6),

$$
\left(1-\varepsilon_{n}\right)\left|z_{n}^{+}-z_{n}^{-}\right| \leq \frac{1}{2}\left(\left|\beta_{n}^{-}\left(z_{n}^{-}\right)\right|+\left|\beta_{n}^{-}\left(z_{n}^{+}\right)\right|+\left|\beta_{n}^{+}\left(z_{n}^{-}\right)\right|+\left|\beta_{n}^{+}\left(z_{n}^{+}\right)\right|\right) .
$$

By the estimates for $\partial \beta / \partial z$ given in Proposition 15, (4.33), we have

$$
\left|\beta_{n}^{ \pm}\left(z_{n}^{ \pm}\right)-\beta_{n}^{ \pm}\left(z_{n}^{*}\right)\right| \leq \varepsilon_{n} \cdot\left|z_{n}^{+}-z_{n}^{-}\right| / 2,
$$

where one may assume that $\varepsilon_{n}$ is the same as in (5.4) and (5.5). Thus we get

$$
\left(1-2 \varepsilon_{n}\right)\left|z_{n}^{+}-z_{n}^{-}\right| \leq\left|\beta_{n}^{+}\left(z_{n}^{*}\right)\right|+\left|\beta_{n}^{-}\left(z_{n}^{*}\right)\right|,
$$

which, in view of (5.1), implies (5.7). Lemma 20 is proved.

5.1. Estimates of $\gamma_{n}$ from below. In the previous Lemma 20 were obtained estimates of $\gamma_{n}$ from above in terms of $\left|\beta_{n}^{ \pm}\right|$. The next statement gives estimates of $\gamma_{n}$ from below. (This is an analogue of Lemma 49 in [14].)

LEMMA 21. In the notations of Lemma 20, there exists a sequence $\eta_{n} \downarrow 0$ such that, for large enough $|n|$, if $\gamma_{n} \neq 0$ and $\beta_{n}^{-}\left(z_{n}^{+}\right) \cdot \beta_{n}^{+}\left(z_{n}^{+}\right) \neq 0$, then

$$
\gamma_{n} \geq\left(\frac{2 \sqrt{t_{n}}}{1+t_{n}}-\eta_{n}\right)\left(\left|\beta_{n}^{-}\left(z_{n}^{*}\right)\right|+\left|\beta_{n}^{+}\left(z_{n}^{*}\right)\right|\right),
$$

where

$$
t_{n}=\left|\beta_{n}^{+}\left(z_{n}^{+}\right)\right| /\left|\beta_{n}^{-}\left(z_{n}^{+}\right)\right| .
$$

Remark. The crucial role of the ratio $t_{n}$ has been realized in [11], Section 4, in particular Proposition 8 (see the comment (a) at the beginning of Section 7 below). Since 2003 it has become an essential ingredient in the analysis of eigenvalue pairs $\lambda^{+}, \lambda^{-}$or troikas $\lambda^{+}, \lambda^{-}, \mu$ in the case of complex-valued potentials (or nonsymmetric potentials in the case of Dirac operators); in the present paper see (7.25) in Proposition 34 and (7.28) in Lemma 35.

Other authors follow [11], Section 4, as well but - strangely enough - do not tell that to the reader; see, for example, Lemma 10 in [64]. 
Proof. By Proposition 15, (4.33),

$$
\sup _{\left[z_{n}^{-}, z_{n}^{+}\right]}\left|\partial \beta_{n}^{ \pm} / \partial z\right| \leq \varepsilon_{n}, \quad \varepsilon_{n} \downarrow 0,
$$

where $\left[z_{n}^{-}, z_{n}^{+}\right]$denotes the segment determined by $z_{n}^{-}$and $z_{n}^{+}$. Since

$$
\beta_{n}^{ \pm}(z)-\beta_{n}^{ \pm}\left(z_{n}^{*}\right)=\int_{z}^{z_{n}^{*}} \frac{d}{d z}\left(\beta_{n}^{ \pm}(z)\right) d z
$$

(5.10) implies that

$$
\left|\beta_{n}^{ \pm}(z)-\beta_{n}^{ \pm}\left(z_{n}^{*}\right)\right| \leq \varepsilon_{n}\left|z-z_{n}^{*}\right| \leq \varepsilon_{n}\left|z_{n}^{+}-z_{n}^{-}\right| \quad \text { for } \quad z \in\left[z_{n}^{-}, z_{n}^{+}\right] .
$$

Thus, for $z \in\left[z_{n}^{-}, z_{n}^{+}\right]$,

$$
\left|\beta_{n}^{ \pm}\left(z_{n}^{*}\right)\right|-\varepsilon_{n}\left|z_{n}^{+}-z_{n}^{-}\right| \leq\left|\beta_{n}^{ \pm}(z)\right| \leq\left|\beta_{n}^{ \pm}\left(z_{n}^{*}\right)\right|+\varepsilon_{n}\left|z_{n}^{+}-z_{n}^{-}\right|
$$

By (5.3) and (5.6), we have

$$
\left(1-\varepsilon_{n}\right)\left|z_{n}^{+}-z_{n}^{-}\right| \leq\left|\zeta_{n}^{+}-\zeta_{n}^{-}\right| \leq\left(1+\varepsilon_{n}\right)\left|z_{n}^{+}-z_{n}^{-}\right|
$$

where

$$
\zeta_{n}^{+}=\zeta_{n}\left(z_{n}^{+}\right), \quad \zeta_{n}^{-}=\zeta_{n}\left(z_{n}^{-}\right) .
$$

On the other hand, by (5.2) (i.e., by the basic equation (3.22)), we have

$$
\left(\zeta_{n}^{+}\right)^{2}=\beta_{n}^{+}\left(z_{n}^{+}\right) \beta_{n}^{-}\left(z_{n}^{+}\right), \quad\left(\zeta_{n}^{-}\right)^{2}=\beta_{n}^{+}\left(z_{n}^{-}\right) \beta_{n}^{-}\left(z_{n}^{-}\right)
$$

and therefore,

$$
\left(\zeta_{n}^{+}\right)^{2}-\left(\zeta_{n}^{-}\right)^{2}=\int_{z_{n}^{-}}^{z_{n}^{+}} \frac{d}{d z}\left[\beta_{n}^{+}(z) \beta_{n}^{-}(z)\right] d z .
$$

By (5.10) and (5.11), we have

$$
\sup _{\left[z_{n}^{-}, z_{n}^{+}\right]}\left|\frac{d}{d z}\left[\beta_{n}^{+}(z) \beta_{n}^{-}(z)\right]\right| \leq \varepsilon_{n}\left(\left|\beta_{n}^{+}\left(z_{n}^{*}\right)\right|+\left|\beta_{n}^{-}\left(z_{n}^{*}\right)\right|+2 \varepsilon_{n}\left|z_{n}^{+}-z_{n}^{-}\right|\right) .
$$

In view of (5.12) and (5.13), we get

$$
\begin{aligned}
\left|\zeta_{n}^{+}+\zeta_{n}^{-}\right| & \cdot\left|\zeta_{n}^{+}-\zeta_{n}^{-}\right| \leq \varepsilon_{n}\left(\left|\beta_{n}^{+}\left(z_{n}^{*}\right)\right|+\left|\beta_{n}^{-}\left(z_{n}^{*}\right)\right|+2 \varepsilon_{n}\left|z_{n}^{+}-z_{n}^{-}\right|\right)\left|z_{n}^{+}-z_{n}^{-}\right| \\
& \leq \varepsilon_{n}\left(\left|\beta_{n}^{+}\left(z_{n}^{*}\right)\right|+\left|\beta_{n}^{-}\left(z_{n}^{*}\right)\right|+2 \varepsilon_{n}\left|z_{n}^{+}-z_{n}^{-}\right|\right) \frac{\left|\zeta_{n}^{+}-\zeta_{n}^{-}\right|}{1-\varepsilon_{n}} .
\end{aligned}
$$

Since $\varepsilon_{n} \rightarrow 0$, we may assume that $\varepsilon_{n}<1 / 2$. Then, $1 /\left(1-\varepsilon_{n}\right) \leq 2$, and the latter inequality implies

$$
\left|\zeta_{n}^{+}+\zeta_{n}^{-}\right| \leq 2 \varepsilon_{n}\left(\left|\beta_{n}^{+}\left(z_{n}^{*}\right)\right|+\left|\beta_{n}^{-}\left(z_{n}^{*}\right)\right|\right)+2 \varepsilon_{n}\left|z_{n}^{+}-z_{n}^{-}\right|
$$

By (5.9), we have

$$
\left|\zeta_{n}^{+}\right|=\sqrt{\left|\beta_{n}^{+}\left(z_{n}^{+}\right)\right|\left|\beta_{n}^{-}\left(z_{n}^{+}\right)\right|}=\frac{\sqrt{t_{n}}}{1+t_{n}}\left(\left|\beta_{n}^{+}\left(z_{n}^{+}\right)\right|+\left|\beta_{n}^{-}\left(z_{n}^{+}\right)\right|\right) .
$$

Therefore, by (5.11) ( since $\frac{\sqrt{t}}{1+t} \leq 1 / 2$ for $t \geq 0$ ) we get

$$
\left|\zeta_{n}^{+}\right| \geq \frac{\sqrt{t_{n}}}{1+t_{n}}\left(\left|\beta_{n}^{+}\left(z_{n}^{*}\right)\right|+\left|\beta_{n}^{-}\left(z_{n}^{*}\right)\right|\right)-\varepsilon_{n}\left|z_{n}^{+}-z_{n}^{-}\right| .
$$

Now, from (5.14)-(5.16) it follows that

$$
\left|\zeta_{n}^{+}-\zeta_{n}^{-}\right|=\left|2 \zeta_{n}^{+}-\left(\zeta_{n}^{+}+\zeta_{n}^{-}\right)\right| \geq 2\left|\zeta_{n}^{+}\right|-\left|\zeta_{n}^{+}+\zeta_{n}^{-}\right|
$$




$$
\geq\left(\frac{2 \sqrt{t_{n}}}{1+t_{n}}-2 \varepsilon_{n}\right)\left(\left|\beta_{n}^{+}\left(z_{n}^{*}\right)\right|+\left|\beta_{n}^{-}\left(z_{n}^{*}\right)\right|\right)-4 \varepsilon_{n}\left|z_{n}^{+}-z_{n}^{-}\right| .
$$

By (5.12), this leads to

$$
\left(1+5 \varepsilon_{n}\right)\left|z_{n}^{+}-z_{n}^{-}\right| \geq\left(\frac{2 \sqrt{t_{n}}}{1+t_{n}}-2 \varepsilon_{n}\right)\left(\left|\beta_{n}^{+}\left(z_{n}^{*}\right)\right|+\left|\beta_{n}^{-}\left(z_{n}^{*}\right)\right|\right) .
$$

Taking into account that $(1+5 \varepsilon)^{-1} \geq 1-5 \varepsilon$ and $\frac{2 \sqrt{t_{n}}}{1+t_{n}} \leq 1$, we obtain

$$
\begin{gathered}
\gamma_{n}=\left|z_{n}^{+}-z_{n}^{-}\right| \geq\left(1-5 \varepsilon_{n}\right)\left(\frac{2 \sqrt{t_{n}}}{1+t_{n}}-2 \varepsilon_{n}\right)\left(\left|\beta_{n}^{+}\left(z_{n}^{*}\right)\right|+\left|\beta_{n}^{-}\left(z_{n}^{*}\right)\right|\right) \\
\geq\left(\frac{2 \sqrt{t_{n}}}{1+t_{n}}-7 \varepsilon_{n}\right)\left(\left|\beta_{n}^{+}\left(z_{n}^{*}\right)\right|+\left|\beta_{n}^{-}\left(z_{n}^{*}\right)\right|\right) .
\end{gathered}
$$

Thus (5.8) holds with $\eta_{n}=7 \varepsilon_{n}$. Lemma 21 is proved.

If the potential $v$ is real-valued, then we have the following two-side estimate of $\gamma_{n}$.

TheOREm 22. Suppose $v$ is a periodic real-valued $H^{-1}$ potential, $L$ is the corresponding self-adjoint Hill-Schrödinger operator and $\left(\gamma_{n}\right)$ is the gap sequence of $L$. Then there exists a sequence $\eta_{n} \downarrow 0$ such that, for $n \geq n_{0}(v)$,

$$
\left(1-\eta_{n}\right)\left(\left|\beta_{n}^{-}\left(z_{n}^{*}\right)\right|+\left|\beta_{n}^{+}\left(z_{n}^{*}\right)\right|\right) \leq\left|\gamma_{n}\right| \leq\left(1+\eta_{n}\right)\left(\left|\beta_{n}^{-}\left(z_{n}^{*}\right)\right|+\left|\beta_{n}^{+}\left(z_{n}^{*}\right)\right|\right)
$$

This result is known in the case of $L^{2}$-potentials (see Theorem 8 in $[\mathbf{9}]$, or Theorem 50 in $[\mathbf{1 4}])$.

Proof. The right inequality in (5.17) has been proved in Lemma 20 for arbitrary (complex-valued) potentials.

Since $L$ is self-adjoint, we know, by Part (b) of Lemma 9, that

$$
\left|\beta_{n}^{+}\left(z_{n}^{+}\right)\right|=\left|\beta_{n}^{-}\left(z_{n}^{+}\right)\right| \text {. }
$$

If $\left|\beta_{n}^{+}\left(z_{n}^{+}\right)\right|=\left|\beta_{n}^{-}\left(z_{n}^{+}\right)\right| \neq 0$ and $\gamma_{n} \neq 0$, then the left inequality in (5.17) follows immediately from Lemma 21 .

If $\left|\beta_{n}^{+}\left(z_{n}^{+}\right)\right|=\left|\beta_{n}^{-}\left(z_{n}^{+}\right)\right|=0$ for some $n$, then $\lambda_{n}^{+}=\lambda^{0}+z_{n}^{+}$is an eigenvalue of geometric multiplicity 2 of the operator $P^{0} L^{0} P^{0}+S\left(\lambda^{+}\right): E^{0} \rightarrow E^{0}$. Therefore, by Remark $7, \lambda_{n}^{+}$is an eigenvalue of geometric multiplicity 2 of the operator $L$, so $\gamma_{n}=0$ and $z_{n}^{*}=z_{n}^{+}$. Thus (5.17) holds.

If $\gamma_{n}=0$ for some $n$, then (since $L$ is self-adjoint) $\lambda_{n}^{+}$is an eigenvalue of $L$ of geometric multiplicity 2 . Therefore, by Remark $7, \lambda_{n}^{+}$is an eigenvalue of geometric multiplicity 2 of the operator $P^{0} L^{0} P^{0}+S\left(\lambda^{+}\right)$. Then the off-diagonal entries of the matrix representation $S\left(\lambda_{n}^{+}\right)$are zeros, i.e., we have $\beta_{n}^{+}\left(z_{n}^{+}\right)=\beta_{n}^{-}\left(z_{n}^{+}\right)=0$ and (5.17) becomes trivial because $z_{n}^{*}=z_{n}^{+}$. Theorem 22 is proved.

Remark. We used to write the Fourier expansion of a potential $v$ in the form $v \sim \sum_{m \in 2 \mathbb{Z}} V(m) e^{i m x}$. Now, for convenience, we set

$$
v_{k}=V(2 k), \quad k \in \mathbb{Z},
$$

and define, for every weight $\Omega=(\Omega(k))_{k \in \mathbb{Z}}$,

$$
\|v\|_{\Omega}^{2}=\sum_{k \in \mathbb{Z}}\left|v_{k}\right|^{2}(\Omega(k))^{2} .
$$


The next theorem generalizes a series of results about asymptotic behavior of $\gamma_{n}$ (see and compare Theorem 41 in [13] in the case of $L^{2}$-potentials $v$ ).

Theorem 23. Suppose $L=L^{0}+v(x), L^{0}=-d^{2} / d x^{2}$, is a periodic HillSchrödinger operator on $I=[0, \pi]$ with $H^{-1}(I)$-potential $v(x)=\sum_{k \in \mathbb{Z}} v_{k} e^{2 k i x}$. Then, for $n>n_{0}(v)$, the operator $L$ has in the disc of center $n^{2}$ and radius $n / 4$ exactly two (counted with their algebraic multiplicity) periodic (for even $n$ ), or anti-periodic (for odd $n$ ) eigenvalues $\lambda_{n}^{+}$and $\lambda_{n}^{-}$. Moreover, for each weight $\Omega=(\Omega(k))_{k \in \mathbb{Z}}$ of the form

$$
\Omega(k)=\tilde{\Omega}(k) / k, \quad k \neq 0,
$$

where $\tilde{\Omega}$ is a submultiplicative weight, we have

$$
\sum_{k \in \mathbb{Z}}\left|v_{k}\right|^{2}(\Omega(k))^{2}<\infty \Rightarrow \sum_{n>n_{0}(v)}\left|\gamma_{n}\right|^{2}(\Omega(n))^{2}<\infty,
$$

where $\gamma_{n}=\lambda_{n}^{+}-\lambda_{n}^{-}$. Moreover,

$$
\sum_{n>n_{0}(v)}\left|\gamma_{n}\right|^{2}(\Omega(n))^{2} \leq C_{1}\|v\|_{\Omega}^{4}+4\|v\|_{\Omega}^{2}
$$

where $C_{1}=C_{1}(\Omega)$.

Proof. By Lemma 20, we have, for $n>n_{0}(v)$,

$$
\left|\gamma_{n}\right| \leq 2\left(\left|\beta_{n}^{-}\left(v ; z_{n}^{*}\right)\right|+\left|\beta_{n}^{+}\left(v ; z_{n}^{*}\right)\right|\right),
$$

where $z_{n}^{*}=\frac{\lambda_{n}^{-}+\lambda_{n}^{+}}{2}-n^{2}$.

Therefore, by Proposition 19, considered with $\Omega_{1} \equiv 1$, we get

$$
\begin{aligned}
& \sum_{n>n_{0}(v)}\left|\gamma_{n}\right|^{2}(\Omega(n))^{2} \leq 4 \sum_{n>n_{0}(v)}\left(\left|\beta_{n}^{-}\left(v ; z_{n}^{*}\right)\right|+\left|\beta_{n}^{+}\left(v ; z_{n}^{*}\right)\right|\right)^{2}(\Omega(n))^{2} \\
& \left.\leq 8 \sum_{n>n_{0}(v)}\left(\left|\beta_{n}^{-}\left(v ; z_{n}^{*}\right)-v_{-n}\right|+\mid \beta_{n}^{+}\left(v ; z_{n}^{*}\right)-v_{n}\right) \mid\right)^{2}(\Omega(n))^{2} \\
& \quad+2 \sum_{n>n_{0}(v)}\left(\left|v_{-n}\right|+\left|v_{n}\right|\right)^{2}(\Omega(n))^{2} \leq 32 C\|v\|_{\Omega}^{4}+4\|v\|_{\Omega}^{2}<\infty
\end{aligned}
$$

with $C=C(\Omega)$, which completes the proof.

\section{Main results for real-valued potentials}

In this section we present our main results on the relationship between spectral gaps rate of decay and potential smoothness for Hill-Schrödinger operators with real-valued periodic singular potentials. However most of the proofs are carried out for arbitrary potentials (see formulas (6.1)-(6.5), Lemma 24 and Proposition 26).

In Theorems 9 and 10, and Section 5.2 in [9], and Theorem 54 in [14], it is proved that the inverse of the implication (5.21) holds for real-valued $L^{2}$-potentials $v$ and (log-concave) submultiplicative weights $\Omega$. Now we extend this result to the case of singular potentials and a wider class of weights.

By Lemma 5, for each periodic potential

$$
v \in H^{-1}([0, \pi]), \quad v(x)=\sum_{k \in \mathbb{Z}} v_{k} e^{2 i k x},
$$


there exists $n_{0}=n_{0}(v)$ such that the constructions of Section 3 work for $n>n_{0}$. In particular, the numbers $\beta_{n}^{ \pm}(v ; z)$ are well-defined if $n>n_{0}(v)$ and $|z| \leq n$.

We set, for $N>n_{0}(v)$,

$$
\Phi_{N}(v)=\sum_{n>N}\left(\left[\beta_{n}^{-}\left(v ; z_{n}^{*}(v)\right)-v_{-n}\right] e^{-2 i n x}+\left[\beta_{n}^{+}\left(v ; z_{n}^{*}(v)\right)-v_{n}\right] e^{2 i n x}\right),
$$

where $z_{n}^{*}(v)=\frac{\lambda_{n}^{-}(v)+\lambda_{n}^{+}(v)}{2}-n^{2}$. Consider the mapping

$$
A_{N}(v)=v+\Phi_{N}(v),
$$

or

$$
A_{N}(v)=H_{N}(v)+T_{N}(v)
$$

with a "head"

$$
H_{N}(v)=\sum_{n \leq N}\left(v_{-n} e^{-2 i n x}+v_{n} e^{2 i n x}\right)
$$

and a "tail"

$$
T_{N}(v)=\sum_{n>N}\left(\beta_{n}^{-}\left(v ; z_{n}^{*}(v)\right) e^{-2 i n x}+\beta_{n}^{+}\left(v ; z_{n}^{*}(v)\right) e^{2 i n x}\right) .
$$

As a finite sum, $H_{N}(v)$ is in $H(\Omega)$ for any $\Omega$. If $v$ is a real-valued potential in $H^{-1}$ then we have, by (6.2) and Theorem 22 ,

$$
\left(\gamma_{n}\right) \in \ell^{2}(\Omega) \Rightarrow\left(\left|\beta_{n}^{-}\left(z_{n}^{*}\right)\right|+\left|\beta_{n}^{+}\left(z_{n}^{*}\right)\right|\right) \in \ell^{2}(\Omega) \Rightarrow T_{N}(v) \in H(\Omega),
$$

and therefore,

$$
\left(\gamma_{n}\right) \in \ell^{2}(\Omega) \Rightarrow A_{N}(v) \in H(\Omega),
$$

for every weight $\Omega$.

Thus, the inverse of the implication (5.21) will be proved if we show that

$$
A_{N}(v) \in H(\Omega) \Rightarrow v \in H(\Omega) .
$$

If $v$ is a real-valued potential, then the operator $L=L^{0}+v$ is self-adjoint, its periodic and anti-periodic spectra are real, so the numbers $z_{n}^{*}=\frac{1}{2}\left(\lambda_{n}^{+}-\lambda_{n}^{-}\right)-n^{2}$ are real.

Therefore, by (3.33) and Lemma 9 we have

$$
\beta_{n}^{-}\left(v ; z_{n}^{*}\right)=\overline{\beta_{n}^{+}\left(v ; z_{n}^{*}\right)} .
$$

Thus, in view of (6.1) and (6.2),

$$
v \text { is real-valued } \Rightarrow T_{N}(v), \Phi_{N}(v), A_{N}(v) \text { are real-valued. }
$$

For each weight $\Omega$ on $\mathbb{Z}$ we denote by $\mathcal{B}_{r}^{\Omega}$ the ball of complex-valued potentials

$$
\mathcal{B}_{r}^{\Omega}=\left\{v \in H(\Omega):\|v\|_{\Omega} \leq r\right\} .
$$

The following lemma plays a crucial role in the proof of the inverse of (5.21).

LEMMA 24. Let $\Omega_{1}$ be a slowly increasing unbounded weight such that

$$
\Omega_{1}(k) \leq C_{1}|k|^{1 / 4} .
$$

Then there exist a sequence of positive numbers $\left(r_{N}\right)_{N \in \mathbb{N}}, r_{N} \nearrow \infty$, and $N^{*}=$ $N^{*}\left(\Omega_{1}\right) \in \mathbb{N}$ such that the mapping $\Phi_{N}$ is well defined on the ball $\mathcal{B}_{3 r_{N}}^{\Omega_{1}}$ for $N>N^{*}$. 
Moreover, if $\Omega=(\Omega(k))_{k \in \mathbb{Z}}$ is a weight of the form

$$
\Omega(k)=\frac{\Omega_{1}(k) \Omega_{2}(k)}{k}, \quad k \in \mathbb{Z},
$$

where $\Omega_{2}$ is a sub-multiplicative weight, then the mapping $\Phi_{N}: \mathcal{B}_{3 r_{N}}^{\Omega} \rightarrow H(\Omega)$ is well defined for $N>N^{*}$ and has the following properties:

$$
\left\|\Phi_{N}\left(v_{1}\right)-\Phi_{N}\left(v_{2}\right)\right\|_{\Omega} \leq \frac{1}{2}\left\|v_{1}-v_{2}\right\|_{\Omega} \quad \text { for } \quad v_{1}, v_{2} \in \mathcal{B}_{r_{N}}^{\Omega}
$$

$$
\begin{gathered}
\frac{1}{2}\left\|v_{1}-v_{2}\right\|_{\Omega} \leq\left\|A_{N}\left(v_{1}\right)-A_{N}\left(v_{2}\right)\right\|_{\Omega} \leq \frac{3}{2}\left\|v_{1}-v_{2}\right\|_{\Omega} \quad \text { for } \quad v_{1}, v_{2} \in \mathcal{B}_{r_{N}}^{\Omega}, \\
A_{N}\left(\mathcal{B}_{r_{N}}^{\Omega}\right) \supset \mathcal{B}_{r_{N} / 2}^{\Omega} .
\end{gathered}
$$

Proof. By Proposition 4, for each $H^{-1}$ periodic potential $v$ there exists $N^{*}=$ $N^{*}(v)$ such that, for $n>N^{*}$, the operator $L=L^{0}+v$ has two (counted according to their algebraic multiplicity, periodic for even $n$, and antiperiodic for odd $n$ ) eigenvalues $\lambda_{n}^{-}(v)$ and $\lambda_{n}^{+}(v)$ in the disk $D_{n}=\left\{z:\left|z-n^{2}\right|<n / 4\right\}$. On the other hand, in view of Lemma 5 , if $N^{*}$ is large enough then all constructions of Section 3 hold.

Moreover, one can choose $N^{*}$ depending only on the weight $\Omega$ and $\|v\|_{\omega_{1}}$, where $\omega_{1}(k)=\Omega(k) / k, k \neq 0$.

Indeed, by the proof of Theorem 21 and Lemma 19, (5.30) in [16], and by the proof of Lemma $5,(3.7)$, it is enough to choose $N^{*}$ so that

$$
\kappa_{n}(v):=C\left(\mathcal{E}_{\sqrt{n}}(q)+\|q\| / \sqrt{n}\right) \leq 1 / 2 \text { for } n \geq N^{*},
$$

where $q=(q(m))$ is defined in (1.4) and $C$ is an absolute constant. So,

$$
\|v\|_{\omega_{1}}^{2}=\sum\left(|q(-k)|^{2}+|q(k)|^{2}\right)\left(\Omega_{1}(k)\right)^{2},
$$

and therefore, since $\Omega_{1}(k)$ is monotone increasing and $\Omega_{1}(k) \geq 1$,

$$
\|q\| \leq\|q\|_{\Omega_{1}}=\|v\|_{\omega_{1}}
$$

and

$$
\mathcal{E}_{m}^{2}(q)=\sum_{|k| \geq m}|q(k)|^{2} \leq \frac{1}{\left(\Omega_{1}(m)\right)^{2}} \sum_{|k| \geq m}|q(k)|^{2}\left(\Omega_{1}(k)\right)^{2} \leq \frac{\|v\|_{\omega_{1}}^{2}}{\left(\Omega_{1}(m)\right)^{2}} .
$$

By $(6.16)-(6.19)$,

$$
\kappa_{n}(v) \leq C\left(\frac{\|v\|_{\omega_{1}}}{\Omega_{1}(\sqrt{n})}+\frac{\|v\|_{\omega_{1}}}{\sqrt{n}}\right)
$$

Therefore, with

$$
r_{N}=\left(\frac{1}{\Omega_{1}(\sqrt{N})}+\frac{1}{\sqrt{N}}\right)^{-1 / 4}
$$

and a proper choice of $N^{*}=N^{*}\left(\Omega_{1},\|v\|_{\omega_{1}}\right)$, we have for $n \geq N \geq N^{*}$

$$
\kappa_{n}(v) \leq C\left(\frac{1}{\Omega_{1}(\sqrt{N})}+\frac{1}{\sqrt{N}}\right)^{1 / 2} \leq \frac{1}{4} \quad \text { if } \quad\|v\|_{\omega_{1}} \leq 3 r_{N} .
$$


But $\|v\|_{\omega_{1}} \leq\|v\|_{\Omega}$, so $\beta_{n}^{ \pm}(v, z), n \geq N$, are well defined if $\|v\|_{\omega_{1}} \leq 3 r_{N}$ and $|z| \leq n / 4$, we have the inequality (4.51) in Proposition 19, which guarantees that

$$
\left\|\Phi_{N}(v)\right\|_{\Omega}^{2} \leq C\left(\Omega_{1}\right)\left(\frac{1}{\Omega_{1}(\sqrt{N})}+\frac{1}{\sqrt{N}}\right)\|v\|_{\Omega}^{4}, \quad v \in B_{3 r_{N}}^{\Omega} \subset B_{3 r_{N}}^{\omega_{1}},
$$

where $C\left(\Omega_{1}\right) \geq 1$.

To explain (6.13), i.e., to show that $\Phi_{N}$ is a contractive mapping with a coefficient $1 / 2$, we estimate its derivative. Fix $v_{1}$ and $w$ such that $\left\|v_{1}\right\|_{\Omega} \leq r_{N}$ and $\|w\|_{\Omega}=1$. The $H(\Omega)$-valued function $\varphi(t)=\Phi_{N}\left(v_{1}+t w\right)$ is analytic in the disc $|t| \leq 2 r_{N}$. Let

$$
b_{N}=\left[C\left(\Omega_{1}\right)\left(\frac{1}{\Omega_{1}(\sqrt{N})}+\frac{1}{\sqrt{N}}\right)\right]^{1 / 2} .
$$

Then, in view of (6.23), we have

$$
\left\|\Phi_{N}(v)\right\|_{\Omega} \leq b_{N}\|v\|_{\Omega}^{2} \leq b_{N} \cdot 9 r_{N}^{2}, \quad v \in B_{3 r_{N}}^{\Omega} .
$$

The Cauchy inequality and (6.21) imply

$$
\begin{gathered}
\sup _{|t| \leq r_{N}}\left\|\frac{d}{d t} \Phi_{N}\left(v_{1}+t w\right)\right\|_{\Omega} \leq \frac{1}{r_{N}} 9 b_{N} r_{N}^{2}=9 b_{N} r_{N} \\
\leq 9 \sqrt{C\left(\Omega_{1}\right)}\left(\frac{1}{\Omega_{1}(\sqrt{N})}+\frac{1}{\sqrt{N}}\right)^{1 / 4} \leq \frac{1}{2}
\end{gathered}
$$

for $N \geq N^{*}$ if $N^{*}$ is chosen large enough.

Therefore, if $v_{1}, v_{2} \in B_{r_{N}}^{\Omega}$ and $\left\|v_{1}-v_{2}\right\|_{\Omega} \leq r_{N}$, then we obtain with $w=$ $\left(v_{1}-v_{2}\right) /\left\|v_{1}-v_{2}\right\|_{\Omega}$

$$
\left\|\Phi_{N}\left(v_{1}\right)-\Phi_{N}\left(v_{2}\right)\right\|_{\Omega} \leq \sup _{|t| \leq r_{N}}\left\|\frac{d}{d t} \Phi_{N}\left(v_{1}+t w\right)\right\|_{\Omega} \cdot\left\|v_{1}-v_{2}\right\|_{\Omega} \leq \frac{1}{2}\left\|v_{1}-v_{2}\right\|_{\Omega} .
$$

If $\left\|v_{1}-v_{2}\right\|_{\Omega}>r_{N}$, then (6.26) implies

$\left\|\Phi_{N}\left(v_{1}\right)-\Phi_{N}\left(v_{1}\right)\right\|_{\Omega} \leq\left\|\Phi_{N}\left(v_{1}\right)\right\|_{\Omega}+\left\|\Phi_{N}\left(v_{1}\right)\right\|_{\Omega} \leq 2 b_{N} r_{N}^{2} \leq \frac{1}{2} r_{N} \leq \frac{1}{2}\left\|v_{1}-v_{2}\right\|_{\Omega}$.

Of course, in view of (6.2), (6.13) implies (6.14).

Finally, a standard argument shows that (6.13)-(6.14) imply (6.15). Namely, for each $u \in B_{r_{N} / 2}^{\Omega}$ the mapping

$$
v \rightarrow u-\Phi_{N}(v)
$$

takes the ball $B_{r_{N}}^{\Omega}$ into itself because (in view of $\Phi(0)=0$ )

$$
\left\|u-\Phi_{N}(v)\right\|_{\Omega} \leq\|u\|_{\Omega}+\left\|\Phi_{N}(v)-\Phi_{N}(0)\right\|_{\Omega} \leq \frac{1}{2} r_{N}+\frac{1}{2} r_{N}=r_{N} .
$$

Thus, with (6.13), by the contraction mapping principle the (nonlinear) operator (6.27) has a unique fixed point $v_{*} \in B_{r_{N}}^{\Omega}$, i.e., $v_{*}=u-\Phi_{N}\left(v_{*}\right)$, or $A_{N}\left(v_{*}\right)=u$. This completes the proof of Lemma 24 .

REMARK 25. Lemma 24 is formulated and proved for spaces of complex-valued periodic potentials $v \in H^{-1}$. In view of Part (b) of Lemma 9 and (3.31), the formulas (6.1)-(6.10) show immediately that this lemma holds for spaces of realvalued potentials as well. 
Proposition 26. Let $\Omega_{1}$ be a slowly increasing unbounded weight such that

$$
\Omega_{1}(k) \leq C_{1}|k|^{1 / 4}, \quad k \in \mathbb{Z},
$$

and let the weight $\omega_{1}$ be defined by $\omega_{1}(k)=\Omega_{1}(k) / k$ for $k>0$. Suppose $\Omega=$ $(\Omega(k))_{k \in \mathbb{Z}}$ is a weight of the form

$$
\Omega(k)=\frac{\Omega_{1}(k) \Omega_{2}(k)}{k}, \quad k \in \mathbb{Z},
$$

where $\Omega_{2}$ is a sub-multiplicative weight.

(a) If

$$
\frac{\log \Omega_{2}(n)}{n} \searrow 0 \quad \text { as } n \rightarrow \infty
$$

then, for $v \in H\left(\omega_{1}\right)$,

$$
\exists N \quad A_{N}(v) \in H(\Omega) \quad \Rightarrow v \in H(\Omega) .
$$

(b) If $\Omega_{2}$ is a sub-multiplicative weight of exponential type, then

$$
\exists N \quad A_{N}(v) \in H(\Omega) \Rightarrow \exists \varepsilon>0: v \in H\left(e^{\varepsilon|n|}\right) .
$$

Proof. (a) If (6.31) holds, then (see Lemma 47 in [14] - this observation comes from [64], Lemma 9) for each $\varepsilon>0$ the weight

$$
\Omega_{2}^{\varepsilon}(m)=\min \left(e^{\varepsilon|m|}, \Omega_{2}(m)\right)
$$

is sub-multiplicative, and obviously for large enough $|m|$ we have $\Omega_{2}^{\varepsilon}(m)=\Omega_{2}(m)$. Let $\Omega^{\varepsilon}$ be a weight given by

$$
\Omega^{\varepsilon}(k)=\frac{\Omega_{1}(k) \Omega_{2}^{\varepsilon}(k)}{k}, \quad k \neq 0 ;
$$

then it follows $H\left(\Omega^{\varepsilon}\right)=H(\Omega)$.

Next we use the constructions and notations of Lemma 24. If $v \in H\left(\omega_{1}\right)$, then

$$
\|v\|_{\omega_{1}}<r_{N} / 8
$$

for large enough $N>N^{*}\left(\Omega_{1},\|v\|_{\omega_{1}}\right)$. We choose $N$ so that (6.32) holds and set

$$
w:=A_{N}(v)=v+\Phi_{N}(v) .
$$

Then, by (6.13) (with $\Omega_{2} \equiv 1$ in (6.12)), we have

$$
\left\|\Phi_{N}(v)\right\|_{\omega_{1}} \leq \frac{1}{2}\|v\|_{\omega_{1}} \leq \frac{r_{N}}{16}
$$

and therefore,

$$
\|w\|_{\omega_{1}}=\left\|A_{N}(v)\right\|_{\omega_{1}} \leq\|v\|_{\omega_{1}}+\left\|\Phi_{N}(v)\right\|_{\omega_{1}} \leq \frac{r_{N}}{4} .
$$

There exists $\varepsilon>0$ such that $\|w\|_{\Omega^{\varepsilon}} \leq r_{N} / 2$. Indeed, let $w(x)=\sum_{k \in \mathbb{Z}} w_{k} \exp (2 i k x)$; choose $N_{1} \in \mathbb{N}$ so that

$$
\sum_{|k|>N_{1}}\left|w_{k}\right|^{2}(\Omega(k))^{2}<\frac{r_{N}^{2}}{16} .
$$

After that, choose $\varepsilon>0$ so that $e^{\varepsilon N_{1}} \leq \sqrt{2}$. Then we have

$$
\Omega_{2}^{\varepsilon}(m) \leq \sqrt{2} \omega_{1}(m) \text { for }|m| \leq N_{1} .
$$


Now (6.35)-(6.37) imply

$\|w\|_{\Omega^{\varepsilon}}^{2} \leq \sum_{|k| \leq N_{1}} 2\left|w_{k}\right|^{2}\left(\omega_{1}(m)\right)^{2}+\sum_{|k|>N_{1}}\left|w_{k}\right|^{2}(\Omega(k))^{2} \leq 2\|w\|_{\omega_{1}}^{2}+\frac{r_{N}^{2}}{16} \leq \frac{r_{N}^{2}}{8}+\frac{r_{N}^{2}}{16}$, and therefore, $\|w\|_{\Omega^{\varepsilon}}<r_{N} / 2$.

By (6.15) in Lemma 24, there exists $\tilde{v} \in \mathcal{B}_{r_{N}}^{\Omega^{\varepsilon}} \subset \mathcal{B}_{r_{N}}^{\omega_{1}}$ such that

$$
A_{N}(\tilde{v})=w=A_{N}(v) \text {. }
$$

On the other hand, by Lemma 24, the restriction of $A_{N}$ on the ball $\mathcal{B}_{r_{N}}^{\omega_{1}}$ is injective. Thus

$$
v=\tilde{v} \in H\left(\Omega^{\varepsilon}\right)=H(\Omega)
$$

(b) If $\Omega_{2}$ is a sub-multiplicative weight of exponential type, i.e.,

$$
\lim _{n \rightarrow \infty} \frac{\log \Omega_{2}(n)}{n}>0,
$$

then, for small enough $\varepsilon>0$,

$$
\Omega_{2}^{\varepsilon}(m)=\min \left(e^{\varepsilon|m|}, \Omega_{2}(m)\right) \equiv e^{\varepsilon|m|}, \quad \Omega^{\varepsilon}(m)=\frac{\Omega_{1}(m)}{m} e^{\varepsilon|m|} .
$$

Thus, the same argument as in (a) shows that $v \in H\left(\Omega^{\varepsilon}\right) \subset H\left(e^{(\varepsilon / 2)|m|}\right)$, which completes the proof of Proposition 26.

Proposition 27. Suppose $\Omega=(\Omega(m))_{m \in \mathbb{Z}}$ is a weight of the form

$$
\Omega(m)=\frac{\omega(m)}{m}, \quad m \neq 0 .
$$

(a) If $\omega$ a sub-multiplicative weight such that

$$
\frac{\log \omega(n)}{n} \searrow 0 \quad \text { as } \quad n \rightarrow \infty
$$

then

$$
\exists N: A_{N}(v) \in \ell^{2}(\mathbb{N}, \Omega) \Rightarrow v \in H(\Omega) .
$$

(b) If $\omega$ is a sub-multiplicative weight of exponential type, i.e.,

$$
\lim _{n \rightarrow \infty} \frac{\log \omega(n)}{n}>0
$$

then

$$
\exists N: A_{N}(v) \in \ell^{2}(\mathbb{N}, \Omega) \Rightarrow \exists \varepsilon>0: v \in H\left(e^{\varepsilon|m|}\right) .
$$

Proof. Let $w:=A_{N}(v)$, and let $\sum_{m \in \mathbb{Z}} w_{m} e^{2 i m x}$ be the Fourier series of $w$.

If $w=A_{N}(v) \in H(\Omega)$, then $\left(\left|w_{m}\right|\right)_{m \in \mathbb{Z}} \in \ell^{2}(\Omega)$. Consider the sequence $x=$ $\left(x_{n}\right)_{n \in \mathbb{N}}$ given by

$$
x_{n}=\left(\frac{1}{n^{2}}\left|v_{-n}\right|^{2}+\frac{1}{n^{2}}\left|v_{n}\right|^{2}+\left(\left|w_{-n}\right|^{2}+\left|w_{n}\right|^{2}\right)(\Omega(n))^{2}\right)^{1 / 2} .
$$

Since $x \in \ell^{2}(\mathbb{N})$, there exists a slowly increasing unbounded weight $\Omega_{1}$ such that $x \in \ell^{2}\left(\mathbb{N}, \Omega_{1}\right)$ (see Lemma 48 in $[\mathbf{1 4}]$ ). We may assume without loss of generality that

$$
\Omega_{1}(n) \leq C_{1}|n|^{1 / 4}
$$

(otherwise, we may replace $\Omega_{1}$ with $\left(\Omega_{1}\right)^{1 / a}$, where $a$ is a suitable constant). 
By the choice of $\Omega_{1}$ we have $A_{N}(v) \in H\left(\Omega_{1} \Omega\right)$ and $v \in H\left(\omega_{1}\right)$, where the weight $\omega_{1}$ is given by $\omega_{1}(m)=\Omega_{1}(m) / m, m>0$. Now, since $H\left(\Omega_{1} \Omega\right) \subset H(\Omega)$, Proposition 27 follows from Proposition 26.

Now we are ready to complete our analysis in the case of real-valued potentials $v \in H^{-1}$

TheOREM 28. Let $L=L^{0}+v(x)$ be the Hill-Schrödinger operator with a periodic real-valued potential $v \in H_{\text {loc }}^{-1}(\mathbb{R}), v(x+\pi)=v(x)$, and let $\gamma=\left(\gamma_{n}\right)$ be its gap sequence. If $\Omega=(\Omega(m))_{m \in \mathbb{Z}}$ is a weight of the form

$$
\Omega(m)=\frac{\omega(m)}{m}, \quad m \neq 0,
$$

where $\omega$ a sub-multiplicative weight such that

$$
\frac{\log \omega(n)}{n} \searrow 0 \quad \text { as } \quad n \rightarrow \infty,
$$

then

$$
\gamma \in \ell^{2}(\mathbb{N}, \Omega) \Rightarrow v \in H(\Omega) .
$$

If $\omega$ is a sub-multiplicative weight of exponential type, i.e.,

$$
\lim _{n \rightarrow \infty} \frac{\log \omega(n)}{n}>0,
$$

then there exists $\varepsilon>0$ such that

$$
\gamma \in \ell^{2}(\mathbb{N}, \Omega) \Rightarrow v \in H\left(e^{\varepsilon|m|}\right) .
$$

Proof. In view of Theorem 22,

$$
\left(\gamma_{n}\right)_{n>N} \in \ell^{2}(\Omega) \Rightarrow \exists N: A_{N}(v) \in H(\Omega) .
$$

Therefore, Theorem 28 follows from Proposition 27.

\section{Complex-valued $H^{-1}$-potentials}

In this section we extend Theorem 28 - with proper adjustments - to the case of complex-valued $H^{-1}$ potentials.

In [11] we did similar "extension" of our results [9] for real $L^{2}$-potentials. We followed the general scheme of $[\mathbf{4 0 , 4 1 , 9}]$ but added two important technical ingredients.

(a) (elementary observation): A $2 \times 2$ matrix $\left(\begin{array}{cc}a & K \\ k & a\end{array}\right)$ has two linearly independent eigenvectors $u_{1}$ and $u_{2}$ if $k K \neq 0$. With the normalization $k K=1$ and $|k| \leq 1$ (otherwise the coordinates in $\mathbb{C}^{2}$ could be interchanged) the angle $\alpha=\alpha\left(u_{1}, u_{2}\right)$ between the eigenvectors $u_{1}=\left(\begin{array}{l}1 \\ k\end{array}\right)$ and $u_{2}=\left(\begin{array}{c}1 \\ -k\end{array}\right)$ is equal to

$$
\arccos \frac{1-|k|^{2}}{1+|k|^{2}}=\arcsin \frac{2 k}{1+|k|^{2}},
$$

so $\alpha \sim 2|k|$ if $|k|<<1$.

(b) (hard analysis) The Riesz projections $P_{n}, P_{n}^{0}$ on $E_{n}$ and $E_{n}^{0}$, respectively, are close in the following sense

$$
\left\|P_{n}-P_{n}^{0}\right\|_{L^{2} \rightarrow L^{2}} \leq \kappa_{n}:=\left\|P_{n}-P_{n}^{0}\right\|_{L^{2} \rightarrow L^{\infty}} \rightarrow 0 .
$$


Remark. In the case of $L^{2}$ potentials

$$
\kappa_{n} \leq \frac{C\|v\|}{n}, \quad n \geq n_{*}
$$

See Proposition 4 in $[\mathbf{1 1}]$ or Proposition 11 in $[\mathbf{1 4}]$. Close estimates may be found in $[77,8,73]$.

Even for $v \in H^{-b}, b \in[0,1)$, the inequalities (7.1) can be proven with

$$
\kappa_{n} \leq C(b) \frac{\left\|v \mid H^{-b}\right\|}{n^{1-b}}, \quad n \geq n_{*},
$$

i.e., these estimates are uniform on the balls in $H^{-b}, b \in[0,1)$.

If $v \in L^{1}$ then

$$
\kappa_{n} \leq C\|v\|_{L^{1}} \frac{\log n}{n} .
$$

This is proven, although not explicitly claimed, in $[\mathbf{7 7}, \mathbf{8}, \mathbf{7 3}]$.

In the case of Dirac operators (see [60], Proposition 8.1 and Corollary 8.6, and [14], Proposition 19, formula (1.165)), estimates like (7.1) do not hold on the balls in $L^{2}$-space of potentials. But for individual potentials or on compacts in $L^{2}$ an analogue of (7.1) holds with

$$
\kappa_{n} \leq C\|v\|\left(\frac{\|v\|}{\sqrt{n}}+\mathcal{E}_{|n| / 2}(w)\right), \quad n \geq n_{*},
$$

where $w$ is an $\ell^{2}$-sequence.

In Appendix, we give estimates of $\left\|P-P_{n}^{0}\right\|_{L^{2} \rightarrow L^{\infty}}$ or even of $\left\|P-P_{n}^{0}\right\|_{L^{1} \rightarrow L^{\infty}}$ in the case of Hill-Schrödinger operators with complex-valued $\pi$-periodic $H^{-1}$ potentials, subject to $\mathrm{Per}^{ \pm}$or Dirichlet boundary conditions. See Proposition 44, Theorem 45 and the inequality (9.84) in Section 9, Appendix.

These facts make possible to preserve the basic structure of the proof in the case of $L^{2}$ potentials: we just need to use Proposition 44, or (9.84). Keeping this in mind we omit details of the proofs (see [14], Section 4) but reproduce the steps and the core statements leading to the proof of the main result.

THEOREM 29. Let $L=L^{0}+v(x)$ be the Hill-Schrödinger operator with a $\pi-$ periodic potential $v \in H_{l o c}^{-1}(\mathbb{R})$. Then, for $n>N(v)$, the operator $L$ has in the disc of center $n^{2}$ and radius $r_{n}=n / 4$ one Dirichlet eigenvalue $\mu_{n}$ and two (counted with their algebraic multiplicity) periodic (for even $n$ ), or antiperiodic (for odd $n$ ) eigenvalues $\lambda_{n}^{+}$and $\lambda_{n}^{-}$.

Let

$$
\Delta_{n}=\left|\lambda_{n}^{+}-\lambda_{n}^{-}\right|+\left|\lambda_{n}^{+}-\mu_{n}\right|, \quad n>N(v)
$$

then, for each weight $\Omega=(\Omega(m))_{m \in \mathbb{Z}}$ of the form

$$
\Omega(m)=\omega(m) / m, \quad m \neq 0,
$$

where $\omega$ is a sub-multiplicative weight, we have

$$
v \in H(\Omega) \Rightarrow\left(\Delta_{n}\right) \in \ell^{2}(\Omega) .
$$

Conversely, if $\omega=(\omega(m))_{m \in \mathbb{Z}}$ is a sub-multiplicative weight such that

$$
\frac{\log \omega(n)}{n} \searrow 0 \quad \text { as } n \rightarrow \infty,
$$


then

$$
\left(\Delta_{n}\right) \in \ell^{2}(\Omega) \Rightarrow v \in H(\Omega) .
$$

If $\omega$ is a sub-multiplicative weight of exponential type, i.e.,

$$
\lim _{n \rightarrow \infty} \frac{\log \omega(n)}{n}>0
$$

then

$$
\left(\Delta_{n}\right) \in \ell^{2}(\Omega) \Rightarrow \exists \varepsilon>0: v \in H\left(e^{\varepsilon|m|}\right) .
$$

Proof. By Proposition 4, for $n>N(v)$, the operator $L$ has in the disc of center $n^{2}$ and radius $r_{n}=n / 4$ one Dirichlet eigenvalue $\mu_{n}$ and two (counted with their algebraic multiplicity) periodic (for even $n$ ), or antiperiodic (for odd $n$ ) eigenvalues $\lambda_{n}^{+}$and $\lambda_{n}^{-}$.

Let $E=E_{n}$ and $E^{0}=E_{n}^{0}$ denote the corresponding 2-dimensional invariant subspace of $L$ and the free operator $L^{0}$ (subject to periodic or antiperiodic bc), and let $P=P_{n}$ and $P^{0}=P_{n}^{0}$ denote the Cauchy-Riesz projections on $E_{n}$ and $E_{n}^{0}$, respectively. In what follows, we fix an $n \in \mathbb{N}$ and consider the corresponding objects like $E=E_{n}, P=P_{n}$ etc, suppressing $n$ in the notations. The subspace $E^{0}=E_{n}^{0}$ has the following standard basis of eigenvectors of $L^{0}$ (corresponding to the eigenvalue $n^{2}$ ):

$$
e^{1}(x)=e^{-i n x}, \quad e^{2}(x)=e^{i n x}, \quad n \in \mathbb{N}
$$

If the restriction of $L$ on $E$ has two distinct eigenvalues, we denote them by $\lambda^{+}$ and $\lambda^{-}$, where $\lambda^{+}$is the eigenvalue which real part is larger, or which imaginary part is larger if the real parts are equal, and set $\gamma=\lambda^{+}-\lambda^{-} .3 \mathrm{~mm}$

Step 1 (analogue of Lemma 59 in [14]).

LEMMA 30. In the above notations, for large enough $n$, there exists a pair of vectors $f, \varphi \in E=E_{n}$ such that

(a) $\|f\|=1,\|\varphi\|=1,\langle f, \varphi\rangle=0$;

(b) $L f=\lambda^{+} f$;

(c) $L \varphi=\lambda^{+} \varphi-\gamma \varphi+\xi f$.

Moreover, with $\varphi^{0}=P^{0} \varphi$, we have

$$
|\xi| \leq 4|\gamma|+2\left\|\left(z^{+}-S\left(\lambda^{+}\right)\right) \varphi^{0}\right\|
$$

and

$$
\left\|\left(z^{+}-S\left(\lambda^{+}\right)\right) \varphi^{0}\right\| \leq 2(|\xi|+|\gamma|),
$$

where $z^{+}=\lambda^{+}-n^{2}$ and $S\left(\lambda^{+}\right): E^{0} \rightarrow E^{0}$ is the operator (3.9) constructed in Lemma 6.

Proof is given in [14], Lemma 59. However, there in all inequalities on pp. 735-736 after (4.9) till the lines $9-10$, p. 736 we need to use (9.84) to guarantee that $\kappa_{n} \rightarrow 0$.

Step 2. In what follows we use Lemma 30 and its notations. Let $f, \varphi \in E$ be the orthonormal pair of vectors constructed in Lemma 30 , and let $f_{1}^{0}, f_{2}^{0}$ and $\varphi_{1}^{0}, \varphi_{1}^{0}$ be the coordinates of $f^{0}=P^{0} f$ and $\varphi^{0}=P^{0} \varphi$ with respect to the basis $\left\{e^{1}, e^{2}\right\}$, i.e.,

$$
f^{0}(x)=f_{1}^{0} e^{1}(x)+f_{2}^{0} e^{2}(x), \quad \varphi^{0}(x)=\varphi_{1}^{0} e^{1}(x)+\varphi_{2}^{0} e^{2}(x) .
$$


Then $L f=\lambda^{+} f$ and, by Lemma 6 , the vector $f^{0}=P^{0} f$ is an eigenvector of the operator $L^{0}+S\left(\lambda^{+}\right): E^{0} \rightarrow E^{0}$ with eigenvalue $\lambda^{+}$. This leads to

$$
\left(\begin{array}{cc}
\zeta^{+} & B^{-} \\
B^{+} & \zeta^{+}
\end{array}\right)\left(\begin{array}{l}
f_{1}^{0} \\
f_{2}^{0}
\end{array}\right)=\left(\begin{array}{c}
\zeta^{+} f_{1}^{0}+B^{-} f_{2}^{0} \\
B^{+} f_{1}^{0}+\zeta^{+} f_{2}^{0}
\end{array}\right)=\left(\begin{array}{l}
0 \\
0
\end{array}\right), \quad\left(\zeta^{+}\right)^{2}=B^{+} B^{-},
$$

where $\left(\begin{array}{cc}\zeta^{+} & B^{-} \\ B^{+} & \zeta^{+}\end{array}\right)$is the matrix representation of the operator $z^{+}-S\left(\lambda^{+}\right)$, so, in view of (3.22) and (3.33),

$$
\zeta^{+}=z^{+}-\alpha_{n}\left(z^{+}\right), \quad B^{ \pm}=\beta_{n}^{ \pm}\left(z^{+}\right), \quad z^{+}=\lambda^{+}-n^{2} .
$$

LEMMA 31. In the above notations, for large enough $n$,

$$
\frac{1}{2}\left(\left|B^{+}\right|+\left|B^{-}\right|\right) \leq\left\|\left(z^{+}-S\left(\lambda^{+}\right)\right) \varphi^{0}\right\| \leq\left(\left|B^{+}\right|+\left|B^{-}\right|\right)
$$

Proof is really given in [14], Lemma 60. However, there in inequalities (4.21), (4.22) and between we need to use (9.84) to guarantee that $\kappa \equiv \kappa_{n} \leq 1 / 2$ for $n \geq n(v)$.

Step 3. Upper bounds for deviations $\left|\mu-\lambda^{+}\right|$.

Now we construct a Dirichlet function $G \in E=E_{n}$ and use it to estimate $\left|\mu-\lambda^{+}\right|$in terms of $\left|B^{-}\right|$and $\left|B^{+}\right|$.

Let $g$ be a unit Dirichlet eigenvector that corresponds to $\mu$, i.e.,

$$
L_{\text {dir }} g=\mu g, \quad\|g\|=1,
$$

and let $P_{d i r}$ be the Cauchy-Riesz projection on the corresponding one-dimensional eigenspace.

LEMMA 32. Under the assumptions of Lemma 30,

(a) there is a vector $G \in E=E_{n}$ of the form

$$
G=a f+b \varphi, \quad\|G\|^{2}=|a|^{2}+|b|^{2}=1,
$$

such that

$$
G(0)=0, \quad G(\pi)=0,
$$

i.e., $G$ is in the domain of $L_{d i r}$.

(b) Moreover, we have

$$
\tau\left(\mu-\lambda^{+}\right)=b \xi\left\langle P_{\text {dir }} f, g\right\rangle-b \gamma\left\langle P_{\text {dir }} \varphi, g\right\rangle,
$$

where $\tau=\tau_{n}$,

$$
1 / 2 \leq|\tau| \leq 2 .
$$

Proof repeats the proof of Lemma 61 (its Hill-Schrödinger part) in [14] but on pp. 739-740 in inequalities (4.33-39) and (4.40-42) again we use (9.84) to guarantee that $\kappa_{n} \rightarrow 0$.

Steps 1-3 lead us to the following

Proposition 33. Under the above assumptions and notations, for large enough $n$, we have

$$
\left|\mu-\lambda^{+}\right| \leq 18|\gamma|+8\left(\left|B^{+}\right|+\left|B^{-}\right|\right)
$$


Proof is given in [14], Proposition 62, where it is explained how the statements of steps 1-3 imply the inequality (7.24).

Step 4.

Proposition 34. For large enough $n$, if

$$
\frac{1}{4}\left|B^{-}\right| \leq\left|B^{+}\right| \leq 4\left|B^{-}\right|
$$

then

$$
\left|\beta_{n}^{-}\left(z_{n}^{*}\right)\right|+\left|\beta_{n}^{+}\left(z_{n}^{*}\right)\right| \leq 2\left|\gamma_{n}\right|,
$$

where $B^{ \pm}=\beta_{n}^{ \pm}\left(z^{+}\right)$and $z_{n}^{*}=\frac{\lambda_{n}^{+}+\lambda_{n}^{-}}{2}-n^{2}$ in the case of simple eigenvalues, $z_{n}^{*}=\lambda_{n}^{+}-n^{2}$ otherwise.

Proof. If (7.25) holds, then either $B^{+}=B^{-}=0$, or $B^{+} B^{-} \neq 0$. If we have $B^{+}=0$ and $B^{-}=0$, then $\lambda^{+}$is an eigenvalue of geometric multiplicity 2 of the operator $P^{0} L^{0} P^{0}+S\left(\lambda^{+}\right)$. That may happen, by Remark 7 , if and only if $\lambda^{+}$is an eigenvalue of geometric multiplicity 2 of the operator $L$ also. But then $\gamma_{n}=0, z_{n}^{*}=z_{n}^{+}$, and therefore, (7.26) holds.

If $B^{+} B^{-} \neq 0$ and $\gamma_{n} \neq 0$, then the claim follows from Lemma 21 because $(7.25)$ implies $t=\left|B^{+}\right| /\left|B^{-}\right| \in[1 / 4,4]$, and therefore, $2 \sqrt{t} /(1+t) \geq 4 / 5$.

Finally, let us consider the case, where $B^{+} B^{-} \neq 0$ but $\gamma_{n}=0$. By Lemma 6 , if $z \in D=\{w:|w|<n / 4\}$, then the point $\lambda=n^{2}+z$ is an eigenvalue of $L$ if and only if $z$ is a root of the basic equation

$$
h(z):=(\zeta(z))^{2}-\beta^{+}(z) \beta^{-}(z)=0, \quad \zeta(z)=z-\alpha(z) .
$$

Therefore, if $\gamma_{n}=0$, then $z^{+}=\lambda^{+}-n^{2}$ is the only root of the equation $h(z)=0$ on the disc $D$.

Moreover, the root $z^{+}$is of multiplicity 2. Indeed, consider the two equations $z^{2}=0$ and $h(z)=0$ on the disk $D$. In view of Proposition 15 , the maximum values of $\left|\alpha_{n}(z)\right|$ and $\left|\beta_{n}^{ \pm}(z)\right|$ on the circle $\partial D=\{z:|z|=n / 4\}$ do not exceed $n \varepsilon_{n}$, where $\varepsilon_{n} \rightarrow 0$ as $n \rightarrow \infty$. Since

$$
h(z)-z^{2}=-2 \alpha z+\alpha^{2}-\beta^{+} \beta^{-},
$$

we have

$$
\sup _{\partial D}\left|h(z)-z^{2}\right| \leq \sup _{\partial D}\left(2|z||\alpha|+\left|\alpha^{2}\right|+\left|\beta^{+} \beta^{-}\right|\right) \leq\left(\frac{n}{4}\right)^{2} \eta_{n},
$$

where $\eta_{n}=8 \varepsilon_{n}\left(1+4 \varepsilon_{n}\right) \rightarrow 0$.

Therefore, for large enough $n$, we have

$$
\sup _{\partial D}\left|h(z)-z^{2}\right|<\sup _{\partial D}\left|z^{2}\right|
$$

so the Rouche Theorem implies that $z^{+}$is a double root of the equation $h(z)=0$. Thus, the derivative of $h$ vanishes at $z^{+}$, i.e.,

$$
2 \zeta\left(z^{+}\right) \cdot\left(1-\frac{d \alpha}{d z}\left(z^{+}\right)\right)=\frac{d \beta^{+}}{d z}\left(z^{+}\right) \cdot \beta^{-}\left(z^{+}\right)+\beta^{+}\left(z^{+}\right) \cdot \frac{d \beta^{-}}{d z}\left(z^{+}\right) .
$$

By Proposition 15, formula (4.33), we have, for large enough $n$,

$$
\left|\frac{d \alpha}{d z}\left(z^{+}\right)\right| \leq \frac{1}{5}, \quad\left|\frac{d \beta^{ \pm}}{d z}\left(z^{+}\right)\right| \leq \frac{1}{5} .
$$


Therefore, by the triangle inequality, we get

$$
2\left|\zeta^{+}\right|(1-1 / 5) \leq \frac{1}{5}\left(\left|B^{+}\right|+\left|B^{-}\right|\right)
$$

where $\left|\zeta^{+}\right|=\left|\zeta\left(z^{+}\right)\right|=\sqrt{\left|B^{+} B^{-}\right|}$. Thus, the latter inequality implies, in view of $(7.25)$,

$$
8 \leq \sqrt{\left|B^{+} / B^{-}\right|}+\sqrt{\left|B^{-} / B^{+}\right|} \leq 4,
$$

which is impossible. This completes the proof.

Step 5. Next we consider the case that is complementary to (7.25), i.e.,

$$
\text { (a) } 4\left|B^{+}\right|<\left|B^{-}\right| \quad \text { or }(b) \quad 4\left|B^{-}\right|<\left|B^{+}\right| \text {. }
$$

We begin with the following technical statement (which is an analogue of Lemma 9 in $[\mathbf{1 1}])$.

LEMMA 35. If $n$ is large enough and (7.27) holds, then

$$
\frac{1}{4} \leq \frac{|f(0)|}{|\varphi(0)|} \leq 4 \text {. }
$$

Proof is essentially given in [14], pp. 743-744, but again to justify the analogs of the inequalities (4.49) to (4.56) and between in [14], we use Proposition 44 (or Theorem 45) to claim that $\kappa_{n} \rightarrow 0$.

Step 6.

Proposition 36. If (7.27) holds, then we have, for large enough $n$,

$$
\left|B^{+}\right|+\left|B^{-}\right| \leq 36|\gamma|+144\left|\mu-\lambda^{+}\right| \text {. }
$$

Proof with the same disclaimer as above is given in [14], pp. 744-745.

Step $\%$.

TheOREM 37. Let $L=L^{0}+v(x)$ be the Hill-Schrödinger operator with a $\pi-$ periodic potential $v \in H_{\text {loc }}^{-1}(\mathbb{R})$. For large enough $n$, if $\lambda_{n}^{+}, \lambda_{n}^{-}$is the $n$-th couple of periodic (for even $n$ ) or antiperiodic (for odd $n$ ) eigenvalues of $L, \gamma_{n}=\lambda_{n}^{+}-\lambda_{n}^{-}$, and $\mu_{n}$ is the $n$-th Dirichlet eigenvalue of $L$, then

(7.30) $\frac{1}{72}\left(\left|\beta_{n}^{-}\left(z_{n}^{*}\right)\right|+\left|\beta_{n}^{+}\left(z_{n}^{*}\right)\right|\right) \leq\left|\gamma_{n}\right|+\left|\mu_{n}-\lambda_{n}^{+}\right| \leq 58\left(\left|\beta_{n}^{-}\left(z_{n}^{*}\right)\right|+\left|\beta_{n}^{+}\left(z_{n}^{*}\right)\right|\right)$.

Proof. By Proposition 4 (localization of spectra) and Proposition 15,

$$
\sup _{\left[z_{n}^{-}, z_{n}^{+}\right]}\left|\partial \alpha_{n} / \partial z\right| \leq \varepsilon_{n}, \quad \sup _{\left[z_{n}^{-}, z_{n}^{+}\right]}\left|\partial \beta_{n}^{ \pm} / \partial z\right| \leq \varepsilon_{n}, \quad \varepsilon_{n} \downarrow 0,
$$

where $\left[z_{n}^{-}, z_{n}^{+}\right]$denotes the segment with end points $z_{n}^{-}$and $z_{n}^{+}$. Therefore, since $\left|z_{n}^{+}-z_{n}^{*}\right| \leq\left|\gamma_{n}\right|=\left|z_{n}^{+}-z_{n}^{-}\right|$, we have

$$
\left|\beta_{n}^{ \pm}\left(z_{n}^{+}\right)-\beta_{n}^{ \pm}\left(z_{n}^{*}\right)\right| \leq \varepsilon_{n} \cdot\left|\gamma_{n}\right|
$$

By the triangle inequality, it follows, for large enough $n$, that

$$
\left|B^{ \pm}\right|-\frac{1}{2}\left|\gamma_{n}\right| \leq\left|\beta_{n}^{ \pm}\left(z_{n}^{*}\right)\right| \leq\left|B^{ \pm}\right|+\frac{1}{2}\left|\gamma_{n}\right|,
$$

where $B^{ \pm}=\beta_{n}^{ \pm}\left(z_{n}^{+}\right)$. 
In view of (7.31), Propositions 34 and 36 imply the left inequality in (7.30). On the other hand, Lemma 20 yields that, for large enough $n$,

$$
\left|\gamma_{n}\right| \leq 2\left(\left|\beta_{n}^{-}\left(z_{n}^{*}\right)\right|+\left|\beta_{n}^{+}\left(z_{n}^{*}\right)\right|\right) .
$$

Therefore, Proposition 33 and the inequality (7.31) imply the right inequality in (7.30). Theorem 37 is proved.

Step 8. Let $N$ be so large that Theorem 37 holds for $n \geq N$, and let $\Omega=$ $(\Omega(m))_{m \in \mathbb{Z}}$ be a weight of the form

$$
\Omega(m)=\omega(m) / m, \quad m \neq 0,
$$

where $\omega$ is a sub-multiplicative weight. By the right inequality in (7.30), Theorem 37 , we have, for $n>N$,

$$
\begin{gathered}
\Delta_{n}=\left|\gamma_{n}\right|+\left|\mu_{n}-\lambda_{n}^{+}\right| \leq 58\left(\left|\beta_{n}^{-}\left(z_{n}^{*}\right)\right|+\left|\beta_{n}^{+}\left(z_{n}^{*}\right)\right|\right) \\
\leq 58\left(\left|v_{-n}\right|+\left|v_{n}\right|+\left|\beta_{n}^{-}\left(z_{n}^{*}\right)-v_{-n}\right|+\left|\beta_{n}^{+}\left(z_{n}^{*}\right)-v_{n}\right|\right) .
\end{gathered}
$$

Therefore, in view of (4.51) in Proposition 19, or more precisely as in its specification (5.23), we obtain

$$
\sum_{n>N} \Delta_{n}^{2}(\Omega(n))^{2} \leq C \sum_{n>N}\left(\left|v_{-n}\right|^{2}+\left|v_{n}\right|^{2}\right)(\Omega(n))^{2}+C\left(\|v\|_{\Omega}^{4}+\|v\|_{\Omega}^{2}\right)<\infty,
$$

which proves (7.7).

Conversely, suppose $\left(\Delta_{n}\right)_{n \geq N} \in \ell^{2}(\Omega)$ with $\Omega(m)=\omega(m) / m, \quad m \neq 0$, where $\omega$ is a sub-multiplicative weight having the property (7.8) (or, respectively, (7.10)). Then, by the left inequality in (7.30), Theorem 37 , we have

$$
\left(\Delta_{n}\right)_{n \geq N} \in \ell^{2}(\Omega) \Rightarrow\left(\left|\beta_{n}^{-}\left(z_{n}^{*}\right)\right|+\left|\beta_{n}^{+}\left(z_{n}^{*}\right)\right|\right)_{n \geq N} \in \ell^{2}(\Omega) .
$$

This yields, in view of the definition of the mapping $A_{N}$ (see (6.2)-(6.5)), that $A_{N}(v) \in H(\Omega)$ because, for $n>N$, the numbers $\beta_{n}^{ \pm}\left(z_{n}^{*}\right)$ are, respectively, the $\pm n$ th Fourier coefficients of $A_{N}(v)$. Now, by Proposition 27, we get $v \in H(\Omega)$ (or, respectively, $v \in H\left(e^{\varepsilon|m|}\right)$ ), which completes the proof of Theorem 29.

\section{Comments}

1. In his preprint $[\mathbf{6 4}] \mathrm{J}$. Pöschel presented results of $[40,41,9, \mathbf{1 0}, \mathbf{1 1}]$ and made attempts to improve the technical exposition and to ease some assumptions, for example on weight sequences $\Omega$. But its starting point (at least in the case of complex-valued $L^{2}$-potentials) is the family of "alternate gap lengths" which would mimic the properties of Dirichlet eigenvalues. He mentions that Sansuc and Tkachenko (presumably in [67]) considered the quantities $\delta_{n}=\mu_{n}-\tau_{n}$, where $\mu_{n}$ are the Dirichlet eigenvalues and $\tau_{n}=\left(\lambda_{n}^{+}+\lambda_{n}^{-}\right) / 2$ are the midpoints of the spectral gaps, and then claims: "More generally, one may consider a family of continuously differentiable alternate gap lengths $\delta_{n}: \mathcal{H}^{0} \rightarrow \mathbb{C}$, characterized by the properties that

- $\delta_{n}$ vanishes whenever $\lambda_{n}^{+}=\lambda_{n}^{-}$has also geometric multiplicity 2 , and

- there are real numbers $\xi_{n}$ such that its gradient satisfy

$$
d \delta_{n}=t_{n}+O(1 / n), \quad t_{n}=\cos 2 \pi n\left(x+\xi_{n}\right),
$$

uniformly on bounded subsets of $\mathcal{H}^{0}$. That is,

$$
\left\|d_{q} \delta_{n}-t_{n}\right\|_{0} \leq C_{q}\left(\|q\|_{0}\right) / n
$$


with $C_{\delta}$ depending only on $\|q\|_{0}:=\|q\|_{\mathcal{H}^{0}}$ ".

(In [64], line 10 on page $3, \mathcal{H}^{0}$ is defined as the $L^{2}$-space of complex-valued functions on $[0, \pi]$.)

But such entire functions $\delta_{n}$ do not exist. (It means that many constructions of [64] manipulate with an empty set.) This was well understood by J. Meixner and F. W. Schäfke in the early 1950 's. They explained $[\mathbf{5 5 , 5 6}]$ that the $n$-th Dirichlet eigenvalue $E_{n}(z)$ of the Mathieu operator

$$
L(z) y=-y^{\prime \prime}+z \cos 2 x y, \quad y(0)=y(\pi)=0, \quad E_{n}(0)^{2},
$$

has a finite radius of analyticity.

This phenomenon is very important in understanding and construction of analytic functions used in the papers $[40,41,9,10,11]$

Now, for completeness of our analysis and presentation, we give a proof of the following statement which is a generalization of the Meixner-Schäfke result ([55], Thm 8, Section 1.5).

Proposition 38. Let

$$
v(x)=\sum_{k=1}^{\infty} v_{k} \sqrt{2} \cos 2 k x,
$$

where $\left(v_{k}\right)$ is a real sequence such that

$$
\sum_{k}\left|v_{k}\right|=\sigma<\infty
$$

and

$$
k v_{k} \rightarrow 0 \quad \text { as } k \rightarrow \infty
$$

Then the $n$-th Dirichlet eigenvalue $E_{n}(z)$ of the operator

$$
L(z) y=-y^{\prime \prime}+z v(x) y, \quad y(0)=y(\pi)=0, \quad E_{n}(0)^{2}
$$

is analytic in a neighborhood of $z=0$, and the radius of convergence $R_{n}$ of its Taylor series about $z=0$ satisfies, for large enough $n$,

$$
R_{n} \leq C n^{2}, \quad C=\frac{32 \sqrt{2} \sigma}{\|v\|^{2}} .
$$

Proof. It is well known (see [44], Sections 7.2 and 7.3) that the function $E_{n}(z)$ is analytic in a neighborhood of 0 .

Let

$$
E_{n}(z)=n^{2}+\sum_{1}^{\infty} a_{k}(z) z^{k}
$$

be its Taylor series expansion about $z=0$, and let $R_{n}$ be the radius of convergence of (8.5).

Proof by the Meixner-Schäfke scheme has two independent (to some extent) parts:

(A) the estimates from above of the Taylor coefficient $a_{2}(n)$ of $E_{n}(z)$, or, more generally, $a_{k}(n), k \geq 3$, in terms of the radius $R_{n}$;

(B) the estimates from below of $a_{2}(n)$, or, more generally, $a_{k}(n), k \geq 3$. 
Part A. Under the condition (8.2) the potential $v$ is a bounded (continuous) function, so the multiplier operator $V: f \rightarrow v f$ is bounded in $L^{2}([0, \pi])$ and

$$
\|V\| \leq \sigma
$$

Let us assume that $E_{n}(z)$ is well defined analytic function of $z$ in a disc $D_{r}=$ $\{z:|z| \leq r\}$. Then we have, with $f \in \operatorname{Dom}(L)$ depending on $z$,

$$
\left(L^{0}+z V\right) f=E_{n}(z) f, \quad\|f\|=1,
$$

and therefore,

$$
E_{n}(z)=\left\langle L^{0} f, f\right\rangle+z\langle V f, f\rangle .
$$

Since the operator $L^{0}$ is self-adjoint, we have

$$
\operatorname{Im} E_{n}(z)=\operatorname{Im}(z\langle V f, f\rangle) .
$$

From (8.6) and (8.9) it follows that

$$
\left|\operatorname{Im}\left(E_{n}(z)-n^{2}\right)\right|=\left|\operatorname{Im} E_{n}(z)\right| \leq \sigma r .
$$

Since $E_{n}(0)^{2}$, in view of (8.10) we have

$$
\frac{1}{2 \pi} \int_{0}^{2 \pi}\left|\operatorname{Re}\left(E_{n}\left(r e^{i t}\right)-n^{2}\right)\right|^{2} d t=\frac{1}{2 \pi} \int_{0}^{2 \pi}\left|\operatorname{Im}\left(E_{n}\left(r e^{i t}\right)-n^{2}\right)\right|^{2} d t \leq \sigma^{2} r^{2}
$$

which implies, by the Cauchy inequality,

$$
\frac{1}{2 \pi} \int_{0}^{2 \pi}\left|E_{n}\left(r e^{i t}\right)-n^{2}\right| d t \leq\left(\frac{1}{2 \pi} \int_{0}^{2 \pi}\left|E_{n}\left(r e^{i t}\right)-n^{2}\right|^{2} d t\right)^{1 / 2} \leq \sqrt{2} \sigma r
$$

Therefore, the Cauchy formula yields

$$
\left|a_{2}(n)\right|=\left|\frac{1}{2 \pi i} \int_{\partial D_{r}} \frac{E_{n}(z)-n^{2}}{z^{3}} d z\right| \leq \frac{1}{2 \pi r^{2}} \int_{0}^{2 \pi}\left|\left(E_{n}\left(r e^{i t}\right)-n^{2}\right)\right| d t \leq \frac{\sqrt{2} \sigma}{r} .
$$

Since this inequality holds for every $r<R_{n}$, we get

$$
\left|a_{2}(n)\right| \leq \frac{\sqrt{2} \sigma}{R_{n}}
$$

so whenever $a_{2}(n) \neq 0$ it implies that $R_{n}$ is finite and

$$
R_{n} \leq \frac{\sqrt{2} \sigma}{\left|a_{2}(n)\right|}
$$

Remark. Of course, an analogue of (8.13) could be derived for any $k>2$. The Cauchy formula and (8.11) imply, for each $r<R_{n}$,

$$
\left|a_{k}(n)\right|=\left|\frac{1}{2 \pi i} \int_{\partial D_{r}} \frac{E_{n}(z)-n^{2}}{z^{k+1}} d z\right| \leq \frac{1}{2 \pi r^{k}} \int_{0}^{2 \pi}\left|\left(E_{n}\left(r e^{i t}\right)-n^{2}\right)\right| d t \leq \frac{\sqrt{2} \sigma}{r^{k-1}},
$$

So

$$
R_{n} \leq\left(\frac{\sqrt{2} \sigma}{\left|a_{k}(n)\right|}\right)^{1 /(k-1)} \quad \text { if } \quad a_{k}(n) \neq 0
$$

Part $B$. To make the inequality (8.13) meaningful as a tool to evaluate $R_{n}$ we need to estimate $\left|a_{2}(n)\right|$ from below. Let us follow the Raleigh-Schrödinger procedure. 
It is well known (e.g., see [44], Section 7.3, in particular (7.3.3)) that there exists an analytic family of eigenvectors

$$
\varphi(z)=\varphi_{0}+z \varphi_{1}+z^{2} \varphi_{2}+\cdots, \quad|z|<\rho<<1,
$$

with

$$
\varphi_{0}=\sqrt{2} \sin n x
$$

Therefore, by (8.5),

(8.17)

$\left(L^{0}+z V\right)\left(\varphi_{0}+z \varphi_{1}+z^{2} \varphi_{2}+\cdots\right)=\left(a_{0}+a_{1} z+a_{2} z^{2}+\cdots\right)\left(\varphi_{0}+z \varphi_{1}+z^{2} \varphi_{2}+\cdots\right)$,

so we have

$$
\begin{gathered}
L^{0} \varphi_{0}=a_{0} \varphi_{0}, \quad a_{0}=n^{2}, \\
L^{0} \varphi_{1}+V \varphi_{0}=a_{0} \varphi_{1}+a_{1} \varphi_{0}, \\
L^{0} \varphi_{2}+V \varphi_{1}=a_{0} \varphi_{2}+a_{1} \varphi_{1}+a_{2} \varphi_{0} .
\end{gathered}
$$

Let us notice that if $g \in \operatorname{Dom}\left(L^{0}\right)=\operatorname{Dom}\left(L^{0}+z V\right)$, then we have

$$
\left\langle\left(L^{0}-a_{0}\right) g, \varphi_{0}\right\rangle=\left\langle g,\left(L^{0}-a_{0}\right) \varphi_{0}\right\rangle=0 .
$$

Therefore, taking the scalar product of both sides of (8.19) with $\varphi_{0}$, we get $(8.22)$

$$
a_{1}=\left\langle V \varphi_{0}, \varphi_{0}\right\rangle=\frac{1}{\pi} \int_{0}^{\pi} v(x) 2 \sin ^{2} n x d x=\frac{1}{\pi} \int_{0}^{\pi} v(x)(1-\cos 2 n x) d x=-\frac{v_{n}}{\sqrt{2}} .
$$

We rewrite (8.19) as

$$
\left(a_{0}-L^{0}\right) \varphi_{1}=\left(V-a_{1}\right) \varphi_{0}
$$

this implies

$$
\varphi_{1}=b_{1} \varphi_{0}+\hat{R}\left(a_{0}\right)\left(V-a_{1}\right) \varphi_{0},
$$

where $b_{1}$ is an unknown constant and

$$
\hat{R}\left(a_{0}\right)(\sin k x)= \begin{cases}0, & k, \\ \frac{1}{n^{2}-k^{2}} \sin k x, & k \neq n .\end{cases}
$$

The next step will give us the value of $a_{2}(n)$. In view of (8.21), a multiplication of both sides of $(8.20)$ by $\varphi_{0}$ leads to

$$
a_{2}(n)=\left\langle\left(V-a_{1}\right) \varphi_{1}, \varphi_{0}\right\rangle .
$$

The first term $b_{1} \varphi_{0}$ in (8.24) is not known but (8.22) yields

$$
\left\langle\left(V-a_{1}\right) b_{1} \varphi_{0}, \varphi_{0}\right\rangle=b_{1}\left\langle\left(V-a_{1}\right) \varphi_{0}, \varphi_{0}\right\rangle=0 .
$$

Therefore,

$$
a_{2}=\left\langle\left(V-a_{1}\right) \hat{R}\left(a_{0}\right)\left(V-a_{1}\right) \varphi_{0}, \varphi_{0}\right\rangle=\sum_{k \neq n} \frac{1}{n^{2}-k^{2}}\left(g_{k}\right)^{2},
$$

where $g_{k}$ are the Fourier coefficients of the function $g=\left(V-a_{1}\right) \varphi_{0}$, i.e.,

$$
g_{k}=\frac{1}{\pi} \int_{0}^{\pi} g(x) \sqrt{2} \sin k x d x, \quad k \in \mathbb{N} .
$$


By (8.1),

(8.28) $V \varphi_{0}=\sum_{k} v_{k} \sqrt{2} \cos 2 k x \sqrt{2} \sin n x=\sum_{k} v_{k}(\sin (n+2 k) x+\sin (n-2 k) x)$.

Therefore, by (8.22), if $n$ is even, $n=2 m$, we have

$$
\left(V-a_{1}\right) \varphi_{0}=\frac{1}{\sqrt{2}} \sum_{p \in \mathbb{N} \backslash\{m\}}\left(v_{|p-m|}-v_{p+m}\right) \sqrt{2} \sin 2 p x,
$$

and if $n$ is odd, $n=2 m-1$,

$$
\left(V-a_{1}\right) \varphi_{0}=\frac{1}{\sqrt{2}} \sum_{p \in \mathbb{N} \backslash\{m\}}\left(v_{|p-m|}-v_{p+m-1}\right) \sqrt{2} \sin (2 p-1) x .
$$

Now (8.27) implies

$$
a_{2}(n)=\frac{1}{8} \sum_{p \in \mathbb{N} \backslash\{m\}} \frac{1}{m^{2}-p^{2}}\left(v_{|p-m|}-v_{p+m}\right)^{2}, \quad n=2 m,
$$

and

$$
a_{2}(n)=\frac{1}{8} \sum_{p \in \mathbb{N} \backslash\{m\}} \frac{1}{(m-p)(m+p-1)}\left(v_{|p-m|}-v_{p+m-1}\right)^{2}, \quad n=2 m-1 .
$$

Of course, these formulas could be useful for different purposes, so let us state the following.

Proposition 39. Assume that the potential $v(x)=\sum_{k} v_{k} \cos 2 k x$ is continuous on $[0, \pi]$. Then the operator $L=-d^{2} / d x^{2}+z V$ subject to Dirichlet boundary conditions has, for $|z|<\rho$, simple eigenvalues $E_{n}(z)$ which are analytic functions, where $\rho>0$ does not depend of $n$. Moreover

$$
E_{n}(0)=n^{2}, \quad E^{\prime}(0)=-v_{n} / \sqrt{2}, \quad E^{\prime \prime}(0)=a_{2}(n) / 2,
$$

where $a_{2}(n)$ is given by (8.31) and (8.32).

Now we use (8.31) and (8.32) to estimate $\left|a_{2}(n)\right|$ from below. We present details for the case of even $n=2 m$ only. For odd $n$ technical details are the same.

Analysis of quadratic forms given by the sums in (8.31) and (8.32) could give many examples of potentials (sequences $\left(v_{k}\right)$ ) with specific properties. To prove our main Proposition 38 we will use the following.

LEMmA 40. Let $\left(v_{k}\right), k \in \mathbb{N}$, be a real $\ell^{2}$-sequence such that, for sufficiently large $k$,

$$
\left|v_{k}\right| \leq \delta / k, \quad 0<\delta \leq\|v\| / 15 .
$$

Then we have, for sufficiently large $n$,

$$
\left|a_{2}(n)\right| \geq \frac{\|v\|^{2}}{32 n^{2}}
$$

Proof. Consider the case $n=2 m$. By (8.31), with $k=|m-p|$, we have

$$
8 a_{2}(n)=\sum_{k=1}^{m-1} \frac{1}{k(2 m-k)}\left(v_{k}-v_{2 m-k}\right)^{2}-\sum_{k=1}^{\infty} \frac{1}{k(2 m+k)}\left(v_{k}-v_{2 m+k}\right)^{2} .
$$


Therefore,

$$
\sum_{k=m}^{\infty} \frac{1}{k(2 m+k)}\left(v_{k}-v_{2 m+k}\right)^{2} \leq \frac{1}{3 m^{2}} \sum_{k=m}^{\infty}\left(2\left|v_{k}\right|^{2}+2\left|v_{2 m+k}\right|^{2}\right) \leq \frac{4}{3 m^{2}}\left(\mathcal{E}_{m}(v)\right)^{2},
$$

where

$$
\mathcal{E}_{m}(v):=\left(\sum_{k=m+1}^{\infty}\left|v_{k}\right|^{2}\right)^{1 / 2} \rightarrow 0 \quad m \rightarrow \infty
$$

On the other hand,

$$
\sum_{k=1}^{m-1} \frac{1}{k(2 m-k)}\left(v_{k}-v_{2 m-k}\right)^{2}-\sum_{k=1}^{m-1} \frac{1}{k(2 m+k)}\left(v_{k}-v_{2 m+k}\right)^{2}=\sum_{j=1}^{5} A_{j},
$$

where

$$
\begin{gathered}
A_{1}=\sum_{k=1}^{m-1} \frac{1}{k}\left(\frac{1}{2 m-k}-\frac{1}{2 m+k}\right)\left(v_{k}\right)^{2}=\sum_{k=1}^{m-1} \frac{2}{(2 m-k)(2 m+k)}\left(v_{k}\right)^{2} \\
\geq \frac{2}{3 m^{2}} \sum_{k=1}^{m-1}\left(v_{k}\right)^{2} \geq \frac{2}{3 m^{2}}\left(\|v\|^{2}-\left(\mathcal{E}_{m}(v)\right)^{2}\right) ; \\
\left|A_{2}\right|=\left|\sum_{k=1}^{m-1} \frac{1}{k(2 m-k)}\left(-2 v_{k} v_{2 m-k}\right)\right| \leq \frac{2 \delta}{m^{2}} \sum_{k=1}^{m-1} \frac{\left|v_{k}\right|}{k} \leq \frac{3\|v\| \delta}{m^{2}}
\end{gathered}
$$

by $(8.34)$, and $\sum\left|v_{k}\right| / k \leq\left(\sum\left|v_{k}\right|^{2}\right)^{1 / 2}\left(\sum 1 / k^{2}\right)^{1 / 2} \leq\|v\| \cdot \frac{\pi}{\sqrt{6}} \leq \frac{3}{2}\|v\|$;

$$
\left|A_{3}\right|=\left|\sum_{k=1}^{m-1} \frac{1}{k(2 m+k)}\left(2 v_{k} v_{2 m+k}\right)\right| \leq \frac{2 \delta}{4 m^{2}} \sum_{k=1}^{m-1} \frac{\left|v_{k}\right|}{k} \leq \frac{3\|v\| \delta}{4 m^{2}}
$$

by the same argument as above;

$$
A_{4}=\sum_{k=1}^{m-1} \frac{1}{k(2 m-k)}\left(v_{2 m-k}\right)^{2} \leq \frac{\delta}{m^{2}} \sum_{k=1}^{m-1} \frac{\left|v_{2 m-k}\right|}{k} \leq \frac{\delta}{m^{2}} \cdot \mathcal{E}_{m}(v) \cdot \frac{\pi}{\sqrt{6}}
$$

by (8.34) and the Cauchy inequality, as in the estimate of $A_{2}$;

$$
\left|A_{5}\right|=\left|-\sum_{k=1}^{m-1} \frac{1}{k(2 m+k)}\left(v_{2 m+k}\right)^{2}\right| \leq \frac{1}{4 m^{2}} \sum_{k=1}^{m-1} \frac{\left|v_{2 m+k}\right|}{k} \leq \frac{\delta}{4 m^{2}} \cdot \mathcal{E}_{m}(v) \cdot \frac{\pi}{\sqrt{6}}
$$

by the same argument as above.

Since $\mathcal{E}_{m}(v) \rightarrow 0$ as $m \rightarrow \infty,(8.36)$, (8.34) and the above inequalities imply, for large enough $m$,

$$
8 a_{2}(2 m) \geq \frac{1}{m^{2}}\left(\frac{1}{2}\|v\|^{2}-\frac{15\|v\| \delta}{4 m^{2}}\right) \geq \frac{\|v\|^{2}}{4 m^{2}} .
$$

This completes the proof of (8.35). 
Now we can complete the proof of Proposition 38. In view of (8.3), the condition (8.34) holds for large enough $k$. Therefore, the inequalities (8.13) and (8.35) hold, so

$$
R_{n} \leq \frac{\sqrt{2} \sigma}{\left|a_{2}(n)\right|} \leq C n^{2}, \quad C=\frac{32 \sqrt{2} \sigma}{\|v\|^{2}} .
$$

2. More comments. In this subsection we will make a few comments on applications of the Banach-Cacciopoli contraction principle (see Section 6) to complement the references in [14], Section 3.5.

In an unpublished 2000 manuscript [58] B. Mityagin used the Banach-Cacciopoli contraction principle to prove density of (complex-valued) finite-zone potentials of Hill operators in $H(\Omega)$-spaces when $\Omega$ is a submultiplicative weight (see Theorem 69 in [14] for a precise statement). His analysis dealt with "tails" (6.5); the "head" was not important but the choice of sufficiently large $N$ to guarantee that $H^{N}$ is contractive has been.

Following the same scheme, P. Djakov and B. Mityagin (see announcement in [59]), and independently (but with extra conditions on $L^{2}$-potentials) B. Grebert and T. Kappeler [31], proved density of finite-zone potentials of 1D Dirac operator (see for an accurate statement [14], Theorem 70). In particular, B. Grebert and T. Kappeler write in [31] (their paper appeared in January 2003 issue of Asymptotic Analysis):

"To prove Theorem 1.1 ... we follow the approach used in [58]: as a set-up we take the Fourier block decomposition introduced first for the Hill operator in $[\mathbf{4 0}, \mathbf{4 1}]$ and used out subsequently for the Zaharov-Shabat operators in $[\mathbf{2 9}, \mathbf{3 0}]$. Unlike in [58] where a contraction mapping argument was used to obtain the density results for the Hill operator, we get a short proof of Theorem 1.1 by applying the inverse function theorem in a straightforward way. As in [58], the main feature of the present proof is that it does not involve any results from the inverse spectral theory." (This is a word-by-word quote from [31] but we changed its reference numbers to fit the reference list of the present paper.)

According to his 2004 preprint [64] J. Pöschel spent a few months in 2003/04 in University of Zürich and had long discussions with T. Kappeler. In [64] he combined "tails" and "heads " into the operator $A_{N}=H_{N}+T_{N} \in(6.3)$. Of course, this does not change the analytic core of the proofs, i.e., the necessity of inequalities which guarantee that $T_{N}$, or $A_{N}$ are contractive, or - B. Grebert and T. Kappeler [31] and J. Pöschel [64] believe that this is a simplification - a version of Implicit Function Theorem could be used instead. T. Kappeler and J. Pöschel [43] claim in 2008 that to use Implicit Function Theorem in the context of spectral gaps was "new functional analytic approach" invented by J. Pöschel. We will not argue with this opinion but mention which elements in [64] we have found really useful for application of the Banach-Cacciopoli contraction principle. Firstly, let us mention the following statement ${ }^{2}$.

\footnotetext{
${ }^{2}$ One of the authors (B.M.) thanks Professor Petr Zabreiko (Belorussian State University, Minsk, Belarus), who reminded him lovely discussions and wonderful atmosphere in the led by Mark Krasnoselski seminar on non-linear functional analysis and differential equations in Voronezh State University, Voronezh, Russia, in 1962-1967.
} 
TRICK 41. (folklore of the 1950's) Let $X_{1} \subset X_{0}$ be two Banach spaces with norms $\|x\|_{0} \leq\|x\|_{1}$. Suppose $F$ is an operator acting in both spaces, $F: X_{j} \rightarrow$ $X_{j}, \quad j=0,1$, so that

$$
\|F(x)-F(y)\|_{j} \leq \frac{1}{2}\|x-y\|_{j}, \quad x, y \in X_{j}, \quad j=0,1 .
$$

If $a \in X_{0}$ and

$$
c=a+F(a) \in X_{1},
$$

then $a \in X_{1}$.

Proof. By (8.37), the operator $y \rightarrow c-F(y)$ is a contraction in $X_{1}$. Therefore, by the Banach-Cacciopoli contraction principle, it has a fixed point $p \in X_{1}$, i.e.,

$$
p=c-F(p) .
$$

By (8.38) and (8.39),

$$
a-p=F(p)-F(a) .
$$

On the other hand, by (8.37) with $j=0$,

$$
\|a-p\|_{0} \leq \frac{1}{2}\|a-p\|_{0}
$$

which yields $a=p$, so $a \in X_{1}$.

We have explained this trick in the case when the operator is defined on the entire spaces $X_{j}, j=0,1$. Of course, as usually, the Banach-Cacciopoli contraction principle is used in Lemma 24 when the operator acts on balls. We follow J. Pöschel [64], when we introduce the weight (6.34) and apply Trick 41. But this "soft" analysis does not help to avoid "hard analysis" of proving that the operators involved are contractive - by 2003, i.e., prior to either [31] or [64], it has been done in $[4 \mathbf{4}, \mathbf{9}, \mathbf{1 1}]$ for Hill operators with $L^{2}$ complex-valued potentials. No surprise, neither [64], no [65] make any claims about 1D periodic Dirac operators (see "hard analysis " in [12] and [14]) or Hill operators with $H^{-1}$-potentials - the latter case is analyzed and done in the present paper.

3. In Introduction - see (1.7),(1.8) - "Sobolev" spaces of functions or weighted sequence spaces are defined by weights $\Omega$. In this paper we consider weights of the form

$$
\Omega(m)=\frac{\omega(m)}{m} \quad \text { for } m \neq 0, \quad \Omega(0)=1,
$$

where $\omega(m)$ is a sub-multiplicative weight such that $\log (\omega(n)) / n$ is monotone decreasing as $n \rightarrow \infty$.

Of course, classical examples of such weights are

$$
\begin{gathered}
\Omega(m)=|m|^{a}, \quad a \geq-1, \\
\Omega(m)=|m|^{s} \exp \left(c|m|^{b}\right), \quad 0<b<1,
\end{gathered}
$$

which give us Sobolev spaces $H^{a}$ or Gevrey spaces $G(b ; s, c)$ correspondingly.

More generally, if $\varphi(x), x \geq 0$, is a concave function such that $\varphi(0)=0$, then the weight

$$
\omega(m)=\exp (\varphi|m|), \quad m \in \mathbb{Z},
$$


is sub-multiplicative, $\log (\omega(n)) / n$ is monotone decreasing as $n \rightarrow \infty$, so we can consider the corresponding Sobolev space $H(\Omega)$ with $\Omega(m)=\omega(m) / m, m \neq 0$. In this general setting a weight $\Omega$ could be chosen "to oscillate" so that the space $H(\Omega)$ does not contain all $C^{\infty}$ (or even Gevrey) functions and at the same time $H(\Omega)$ is not contained in $H^{a}$ for every $a>-1$.

Let us make this remark more formal and precise.

LEMmA 42. Let the functions $a(x), b(x) \in C^{2}([0, \infty))$ satisfy the following conditions:

(i) $a(0)=b(0)=0, \quad a(x)<b(x)$ for $x>0, \quad b(x)-a(x) \rightarrow \infty$ as $x \rightarrow \infty$;

(ii) $a^{\prime}(x), b^{\prime}(x)>0, \quad a^{\prime}(x), b^{\prime}(x) \rightarrow 0$ as $x \rightarrow \infty$;

(iii) $a^{\prime \prime}(x), b^{\prime \prime}(x)<0, \quad x \geq 0$

Then, there is a concave function $g(x)$ and a sequence $c_{k} \uparrow \infty$ such that

$$
a(x) \leq g(x) \leq b(x)
$$

and

$$
g\left(c_{2 k-1}\right)=b\left(c_{2 k-1}\right), \quad g\left(c_{2 k}\right)=a\left(c_{2 k}\right), \quad k=1,2, \ldots
$$

Proof. We construct inductively $c_{k}$ and $g(x)$ so that (8.45) holds and $g(x)$ is linear on the interval $\left[c_{2 k-1}, c_{2 k+1}\right], k \geq 1$.

Choose $c_{1}>0$ large enough to guarantee that

$$
a^{\prime}(x), b^{\prime}(x) \leq 1 / 2 \text { for } x \geq c_{1}
$$

and

$$
b(x)-a(x) \geq 1 \text { for } x \geq c_{1} .
$$

We set $g(x)=b(x)$ for $0 \leq x \leq c_{1}$, and

$$
m_{1}=\inf \left\{m: g\left(c_{1}\right)+m\left(x-c_{1}\right) \geq a(x) \text { for } x \geq c_{1}\right\} .
$$

Concavity of $a(x)$ and the initial condition

$$
g\left(c_{1}\right)=b\left(c_{1}\right)>a\left(c_{1}\right)
$$

guarantee that $m_{1}$ is well-defined by (8.48) and there are uniquely determined points $c_{2}, c_{3}$ such that

$$
m_{1}\left(c_{2}-c_{1}\right)+g\left(c_{1}\right)=a\left(c_{2}\right), \quad m_{1}\left(c_{3}-c_{1}\right)+g\left(c_{1}\right)=b\left(c_{3}\right) .
$$

Therefore, with $g(x)=m_{1}\left(x-c_{1}\right)+g\left(c_{1}\right)$ for $x \in\left[c_{1}, c_{3}\right]$ the condition (8.45) holds for $k=1$.

We continue by induction. Assuming that $c_{1}, \ldots, c_{2 p-1}$ and $g(x), x \leq c_{2 p-1}$, are constructed, we set

$$
m_{p}=\inf \left\{m: g\left(c_{2 p-1}\right)+m\left(x-c_{2 p-1}\right) \geq a(x) \text { for } x \geq c_{2 p-1}\right\} .
$$

Then there are uniquely determined points $c_{2 p}, c_{2 p+1}$ such that

$$
m_{p}\left(c_{2 p}-c_{2 p-1}\right)+g\left(c_{2 p-1}\right)=a\left(c_{2 p}\right), \quad m_{p}\left(c_{2 p+1}-c_{2 p-1}\right)+g\left(c_{2 p-1}\right)=b\left(c_{2 p+1}\right) .
$$

Therefore, with $g(x)=m_{p}\left(x-c_{2 p-1}\right)+g\left(c_{2 p-1}\right)$ for $x \in\left[c_{2 p-1}, c_{2 p+1}\right]$ the condition (8.45) holds for $k=p$.

Since $a\left(c_{2 p}\right) \geq b\left(c_{2 p-1}\right),(8.46)$ and (8.47) imply

$$
1 \leq b\left(c_{2 p}\right)-a\left(c_{2 p}\right) \leq b\left(c_{2 p+1}\right)-b\left(c_{2 p-1}\right) \leq\left(c_{2 p+1}-c_{2 p-1}\right) / 2 .
$$

Therefore, $c_{2 p+1}-c_{2 p-1} \geq 2$, so $c_{k} \rightarrow \infty$. 
Let $a(x), b(x), g(x)$ be the functions from Lemma 42. We are going to define weight sequences using the values of $a(x), b(x), g(x)$ at integer points. Let

$$
n_{k}=\left[c_{k}\right], \quad \text { so } n_{k} \leq c_{k}<n_{k}+1
$$

where $\left(c_{k}\right)$ is the sequence constructed in the proof of Lemma 42 . By its construction, in view of (8.46), the function $g(x)$ is piecewise linear for $x \geq c_{1}$ with positive slopes $m_{p} \leq 1 / 2$. Therefore, the Mean Value Theorem implies

$$
b\left(n_{k}\right)-g\left(n_{k}\right) \geq b\left(c_{k}\right)-a\left(c_{k}\right)-1 / 2 \text { for even } k
$$

and

$$
g\left(n_{k}\right)-a\left(n_{k}\right) \geq b\left(c_{k}\right)-a\left(c_{k}\right)-1 / 2 \text { for odd } k .
$$

Consider the weights

$$
A(m)=\frac{1}{|m|} e^{a(|m|)}, \quad B(m)=\frac{1}{|m|} e^{b(|m|)}, \quad G(m)=\frac{1}{|m|} e^{g(|m|)}, \quad m \neq 0 .
$$

By (i) in Lemma 42, $b(x)-a(x) \rightarrow \infty$ as $x \rightarrow \infty$. Therefore, (8.49) and (8.50) imply

$$
\sup _{n} \frac{B(n)}{G(n)} \geq \sup _{k} \frac{B\left(n_{2 k}\right)}{G\left(n_{2 k}\right)}=\infty, \quad \sup _{n} \frac{G(n)}{A(n)} \geq \sup _{k} \frac{G\left(n_{2 k-1}\right)}{A\left(n_{2 k-1}\right)}=\infty .
$$

We have $A(m) \leq G(m) \leq B(m)$, so in view of $(8.51)$,

$$
H(B) \varsubsetneqq H(G) \varsubsetneqq H(A) \text {. }
$$

LEMMA 43. In the notations of Lemma 42, suppose $f(x) \in C^{2}([0, \infty))$ is a function which satisfies (ii) and (iii) and

$$
\begin{gathered}
a(x) \leq f(x) \leq b(x), \quad x \geq 0 \\
f(x)-a(x) \rightarrow \infty, \quad b(x)-f(x) \rightarrow \infty \quad \text { as } x \rightarrow \infty .
\end{gathered}
$$

Let $F$ be the corresponding the weight sequence $F(m)=|m|^{-1} \exp f(|m|)$ for $m \neq$ $0, F(0)=1$. Then

$$
H(B) \subset H(F) \subset H(A)
$$

and

$$
H(B) \varsubsetneqq H(G) \varsubsetneqq H(A),
$$

but

$$
H(F) \not \subset H(G) \quad \text { and } H(G) \not \subset H(F) .
$$

Proof. Inequalities (8.53) and (8.44) imply the inclusions (8.55), and (8.56) is explained in (8.52). The same argument proves (8.57) because (8.54) implies

$$
\sup _{n} \frac{G(n)}{F(n)} \geq \sup _{k} \frac{G\left(n_{2 k-1}\right)}{F\left(n_{2 k-1}\right)} \geq \sup _{k} \exp \left[-1 / 2+b\left(c_{2 k-1}\right)-f\left(c_{2 k-1}\right)\right]=\infty
$$

and, in a similar way, $\sup _{n} F(n) / G(n)=\infty$. 
Lemmas 42 and 43 give a variety of options to construct weights with prescribed imbedding properties of related Sobolev spaces.

Example 1. For $-1<\alpha<\beta$, let

$$
a(x)=(\alpha+1) \log (x+e), \quad b(x)=(\beta+1) \log (x+e),
$$

and let $g(x), G$ be the corresponding function from Lemma 42 and the weight sequence $G(m)=|m|^{-1} \exp g(|m|)$ for $m \neq 0, G(0)=1$. Then $H^{\beta} \subset H(G) \subset H^{\alpha}$ but we have $H^{\gamma} \not \subset H(G)$ and $H(G) \not \subset H^{\gamma}$ for any $\gamma \in(\alpha, \beta)$.

Example 2. Let

$$
a(x)=\log \log (x+e), \quad b(x)=x / \log (x+e),
$$

and let $g(x), G$ be the corresponding function from Lemma 42 and the weight sequence $G(m)=|m|^{-1} \exp g(|m|)$ for $m \neq 0, G(0)=1$.

Then, for any weight $\Omega$ in (8.41) with $a>-1$ or in (8.42), we have $H(\Omega) \not \subset$ $H(G)$ and $H(G) \not \subset H(\Omega)$. At the same time $H(G) \subset H^{-1}$ and all analytic functions are in $H(G)$.

\section{Appendix:Deviations of Riesz projections of Hill operators with singular potentials}

In this Appendix we show that the deviations $P_{n}-P_{n}^{0}$ of Riesz projections

$$
P_{n}=\frac{1}{2 \pi i} \int_{C_{n}}(z-L)^{-1} d z, \quad C_{n}=\left\{\left|z-n^{2}\right|=n\right\},
$$

of Hill operators $L y=-y^{\prime \prime}+v(x) y, x \in[0, \pi]$, with zero and $H^{-1}$ periodic potentials go to zero as $n \rightarrow \infty$ even if we consider $P_{n}-P_{n}^{0}$ as operators from $L^{1}$ to $L^{\infty}$. This implies that all $L^{p}$-norms are uniformly equivalent on the Riesz subspaces Ran $P_{n}$ (see Theorem 51).

9.1. Preliminaries. Now, in the case of singular potentials, we want to compare the Riesz projections $P_{n}$ of the operator $L_{b c}$, defined for large enough $n$ by the formula

$$
P_{n}=\frac{1}{2 \pi i} \int_{C_{n}}\left(z-L_{b c}\right)^{-1} d z, \quad C_{n}=\left\{\left|z-n^{2}\right|=n\right\},
$$

with the corresponding Riesz projections $P_{n}^{0}$ of the free operator $L_{b c}^{0}$ (although $E_{n}^{0}=\operatorname{Ran}\left(P_{n}^{0}\right)$ maybe have no common nonzero vectors with the domain of $\left.L_{b c}\right)$.

The main result is Theorem 45 (see Subsection 2 below), which claims that

$$
\tilde{\tau}_{n}=\left\|P_{n}-P_{n}^{0}\right\|_{L^{1} \rightarrow L^{\infty}} \rightarrow 0 \text { as } n \rightarrow \infty .
$$

This implies a sort of quantum chaos, namely all $L^{p}$-norms on the Riesz subspaces $E_{n}=\operatorname{Ran} P_{n}$, for bc $=P e r^{ \pm}$or Dir, are uniformly equivalent (see Theorem 51 in Section 9.5).

In our analysis (see [14]) of the relationship between smoothness of a potential $v$ and the rate of decay of spectral gaps and spectral triangles a statement similar to $(9.2)$

$$
\tau_{n}=\left\|P_{n}-P_{n}^{0}\right\|_{L^{2} \rightarrow L^{\infty}} \rightarrow 0 \quad \text { as } n \rightarrow \infty .
$$

was crucial when we used the deviations of Dirichlet eigenvalues from periodic or anti-periodic eigenvalues to estimate the Fourier coefficients of the potentials $v$. But 
if $v \in L^{2}$ it was "easy" (see [11], Section 3, Proposition 4, or [14], Proposition 11). Moreover, those are strong estimates: for $n \geq N\left(\|v\|_{L^{2}}\right)$

$$
\tau_{n} \leq \frac{C}{n}\|v\|_{L^{2}}
$$

where $C$ is an absolute constant. Therefore, in (9.4) only the $L^{2}$-norm is important, so $\tau_{n} \leq C R / n$ holds for every $v$ in an $L^{2}$-ball of radius $R$.

In a more general context, the estimates of sequences (9.2) and (9.3) are related to convergence (or equiconvergence) of spectral decompositions $\sum P_{n} f$ in $L^{p}$ (or $\sum\left(P_{n}-P_{n}^{0}\right) f$ is $\left.L^{\infty}\right)$ under different assumptions on potentials $v$ and functions $f$. For Schrödinger operators, their "spectral decompositions" and structure of projection families see a fundamental paper [27].

Just for comparison let us mention the same type of question in the case of $1 \mathrm{D}$ periodic Dirac operators

$$
M F=i\left(\begin{array}{cc}
1 & 0 \\
0 & -1
\end{array}\right) \frac{d F}{d x}+V F, \quad 0 \leq x \leq \pi
$$

where $V=\left(\begin{array}{ll}0 & p \\ q & 0\end{array}\right), p$ and $q$ are $L^{2}$-functions, and $F=\left(\begin{array}{l}f_{1} \\ f_{2}\end{array}\right)$.

The boundary conditions under consideration are $P e r^{ \pm}$and Dir, where

$$
\text { Per }^{ \pm}: \quad F(\pi)= \pm F(0), \quad \text { Dir }: \quad f_{1}(0)=f_{2}(0), \quad f_{1}(\pi)=f_{2}(\pi) .
$$

Then (see [60] or [14], Section 1.1)

$$
E_{n}^{0}=\left\{\left(\begin{array}{c}
a e^{-i n x} \\
b e^{i n x}
\end{array}\right): a, b \in \mathbb{C}\right\}, \quad n \in \mathbb{Z},
$$

where $n$ is even if $b c=P e r^{+}$and $n$ is odd if $b c=P e r^{-}$, and

$$
E_{n}^{0}=\{c \sin n x, c \in \mathbb{C}\}, \quad n \in \mathbb{N}
$$

if $b c=$ Dir. Then for

$$
Q_{n}=\frac{1}{2 \pi i} \int_{C_{n}}(\lambda-L)^{-1} d \lambda, \quad C_{n}=\{\lambda:|\lambda-n|=1 / 4\},
$$

we have

$$
\rho_{n}(V):=\left\|Q_{n}-Q_{n}^{0}\right\|_{L^{2} \rightarrow L^{\infty}} \rightarrow 0 \quad \text { as } n \rightarrow \infty ;
$$

moreover, for any compact set $K \subset L^{2}$ and $V \in K$, i.e., $p, q \in K$ one can construct a sequence $\varepsilon_{n}(K) \rightarrow 0$ such that $\rho_{n}(V) \leq \varepsilon_{n}(K), V \in K$. This has been proven in [60], Proposition 8.1 and Corollary 8.6; see Proposition 19 in [14] as well. in $L^{2}$

Of course, the norms $\tau_{n}$ in (9.3) are larger than the norms of these operators

$$
t_{n}=\left\|P_{n}-P_{n}^{0}\right\|_{L^{2} \rightarrow L^{2}} \leq \tau_{n}
$$

and better (smaller) estimates for $t_{n}$ are possible. For example, A. Savchuk and A. Shkalikov proved ([71], Sect.2.4) that $\sum t_{n}^{2}<\infty$. This implies (by Bari-Markus theorem - see [28], Ch.6, Sect.5.3, Theorem 5.2) that the spectral decompositions

$$
f=f_{N}+\sum_{n>N} P_{n} f
$$

converge unconditionally. For Dirac operators the Bari-Markus condition is

$$
\sum_{n \in \mathbb{Z},|n|>N}\left\|Q_{n}-Q_{n}^{0}\right\|^{2}<\infty .
$$


This fact (together with the completeness and minimality of the system of Riesz subspaces $\operatorname{Ran} Q_{n}$ ) imply unconditional convergence of the spectral decompositions. This has been proved in $[\mathbf{5 9}, \mathbf{6 0}]$ under the assumption that the potential $V$ is in the Sobolev space $H^{\alpha}, \alpha>1 / 2$ (see [60], Theorem 8.8 for more precise statement). Further results on convergence of spectral expansions for Dirac operators will be given in our forthcoming papers $[\mathbf{1 8}, \mathbf{1 9}]$.

The proof of Theorem 45, or the estimates of norms (9.2), are based on the perturbation theory, which gives the representation

$$
P_{n}-P_{n}^{0}=\frac{1}{2 \pi i} \int_{C_{n}}\left(R(\lambda)-R^{0}(\lambda)\right) d \lambda,
$$

where $R(\lambda)=\left(\lambda-L_{b c}\right)^{-1}$ and $R^{0}(\lambda)$ are the resolvents of $L_{b c}$ and of the free operator $L_{b c}^{0}$, respectively. Often - and certainly in the above mentioned examples where $v \in L^{2}$ - one can get reasonable estimates for the norms $\left\|R(\lambda)-R^{0}(\lambda)\right\|$ on the contour $C_{n}$, and then by integration for $\left\|P_{n}-P_{n}^{0}\right\|$. But now, with $v \in H^{-1}$, we succeed to get good estimates for the norms $\left\|P_{n}-P_{n}^{0}\right\|$ after having integrated term by term the series representation

$$
R-R^{0}=R^{0} V R^{0}+R^{0} V R^{0} V R^{0}+\cdots .
$$

This integration kills or makes more manageable many terms, maybe in their matrix representation. Only then we go to the norm estimates. Technical details of this procedure (Subsection 9.3) is the core of the proof of Theorem 45.

9.2. Main result on the deviations $P_{n}-P_{n}^{0}$. By Proposition 4 (i.e., our Theorem 21 in [16] about spectra localization), the operator $L_{P e r \pm}$ has, for large enough $n$, exactly two eigenvalues (counted with their algebraic multiplicity) inside the disc of radius $n$ about $n^{2}$ (periodic for even $n$ or antiperiodic for odd $n$ ). The operator $L_{D i r}$ has one eigenvalue in these discs for all large enough $n$.

Let $E_{n}$ be the corresponding Riesz invariant subspace, and let $P_{n}$ be the corresponding Riesz projection, i.e.,

$$
P_{n}=\frac{1}{2 \pi i} \int_{C_{n}}(\lambda-L)^{-1} d \lambda
$$

where $C_{n}=\left\{\lambda:\left|\lambda-n^{2}\right|\right\}$. We denote by $P_{n}^{0}$ the Riesz projection that corresponds to the free operator.

Proposition 44. In the above notations, for boundary conditions $b c=P e r^{ \pm}$ or Dir,

$$
\left\|P_{n}-P_{n}^{0}\right\|_{L^{2} \rightarrow L^{\infty}} \rightarrow 0 \quad \text { as } n \rightarrow \infty .
$$

As a matter of fact we will prove a stronger statement.

THEOREM 45. In the above notations, for boundary conditions $b c=P e r^{ \pm}$or Dir

$$
\left\|P_{n}-P_{n}^{0}\right\|_{L^{1} \rightarrow L^{\infty}} \rightarrow 0 \quad \text { as } n \rightarrow \infty .
$$

Proof. We give a complete proof for $b c=P e r^{ \pm}$. If $b c=D i r$ the proof is the same, and only minor changes are necessary due to the fact that in this case the orthonormal system of eigenfunctions of $L^{0}$ is $\{\sqrt{2} \sin n x, n \in \mathbb{N}\}$ (while it is $\{\exp (i m x), m \in 2 \mathbb{Z}\}$ for $b c=P e r^{+}$, and $\{\exp (i m x), m \in 1+2 \mathbb{Z}\}$ for $\left.b c=P e r^{-}\right)$. So, roughly speaking, the only difference is that when working with $b c=P e r^{ \pm}$the 
summation indexes in our formulas below run, respectively, in $2 \mathbb{Z}$ and $1+2 \mathbb{Z}$, while for $b c=D i r$ the summation indexes have to run in $\mathbb{N}$. Therefore, we consider in detail only $b c=P e r^{ \pm}$, and provide some formulas for the case $b c=D i r$.

Let

$$
B_{k m}(n):=\left\langle\left(P_{n}-P_{n}^{0}\right) e_{m}, e_{k}\right\rangle .
$$

We are going to prove that

$$
\sum_{k, m}\left|B_{k m}(n)\right| \rightarrow 0 \quad \text { as } n \rightarrow \infty .
$$

Of course, the convergence of the series in (9.10) means that the operator with the matrix $B_{k m}(n)$ acts from $\ell^{\infty}$ into $\ell^{1}$.

The Fourier coefficients of an $L^{1}$-function form an $\ell^{\infty}$-sequence. On the other hand,

$$
D=\sup _{x, n}\left|e_{n}(x)\right|<\infty .
$$

Therefore, the operators $P_{n}-P_{n}^{0}$ act from $L^{1}$ into $L^{\infty}$ (even into $C$ ) and

$$
\left\|P_{n}-P_{n}^{0}\right\|_{L^{1} \rightarrow L^{\infty}} \leq D^{2} \sum_{k, m}\left|B_{k m}(n)\right| .
$$

Indeed, if $\|f\|_{L^{1}}=1$ and $f=\sum f_{m} e_{m}$, then $\left|f_{m}\right| \leq D$ and

$$
\left(P_{n}-P_{n}^{0}\right) f=\sum_{k}\left(\sum_{m} B_{k m} f_{m}\right) e_{k} .
$$

Taking into account (9.11), we get

$$
\left\|\left(P_{n}-P_{n}^{0}\right) f\right\|_{L^{\infty}} \leq D \sum_{k}\left|\sum_{m} B_{k m} f_{m}\right| \leq D^{2} \sum_{k} \sum_{m}\left|B_{k m}\right|,
$$

which proves (9.12).

In [16], Section 5 , we gave a detailed analysis of the representation

$$
R_{\lambda}-R_{\lambda}^{0}=\sum_{s=0}^{\infty} K_{\lambda}\left(K_{\lambda} V K_{\lambda}\right)^{s+1} K_{\lambda}
$$

where $K_{\lambda}=\sqrt{R_{\lambda}^{0}}-$ see $[\mathbf{1 6}],(5.13-14)$ and what follows there. By (9.5),

$$
P_{n}-P_{n}^{0}=\frac{1}{2 \pi i} \int_{C_{n}} \sum_{s=0}^{\infty} K_{\lambda}\left(K_{\lambda} V K_{\lambda}\right)^{s+1} K_{\lambda} d \lambda .
$$

if the series on the right converges. Thus

$$
\left\langle\left(P_{n}-P_{n}^{0}\right) e_{m}, e_{k}\right\rangle=\sum_{s=0}^{\infty} \frac{1}{2 \pi i} \int_{C_{n}}\left\langle K_{\lambda}\left(K_{\lambda} V K_{\lambda}\right)^{s+1} K_{\lambda} e_{m}, e_{k}\right\rangle d \lambda
$$

so we have

$$
\sum_{k, m}\left|\left\langle\left(P_{n}-P_{n}^{0}\right) e_{m}, e_{k}\right\rangle\right| \leq \sum_{s=0}^{\infty} A(n, s),
$$


where

$$
A(n, s)=\sum_{k, m}\left|\frac{1}{2 \pi i} \int_{C_{n}}\left\langle K_{\lambda}\left(K_{\lambda} V K_{\lambda}\right)^{s+1} K_{\lambda} e_{m}, e_{k}\right\rangle d \lambda\right|
$$

By the matrix representation of the operators $K_{\lambda}$ and $V$ (see more details in [16], (5.15-22)) it follows that

$$
\left\langle K_{\lambda}\left(K_{\lambda} V K_{\lambda}\right) K_{\lambda} e_{m}, e_{k}\right\rangle=\frac{V(k-m)}{\left(\lambda-k^{2}\right)\left(\lambda-m^{2}\right)}, \quad k, m \in n+2 \mathbb{Z},
$$

for $b c=P e r^{ \pm}$, and

$$
\left\langle K_{\lambda}\left(K_{\lambda} V K_{\lambda}\right) K_{\lambda} e_{m}, e_{k}\right\rangle=\frac{|k-m| \tilde{q}(|k-m|)-(k+m) \tilde{q}(k+m)}{\sqrt{2}\left(\lambda-k^{2}\right)\left(\lambda-m^{2}\right)}, \quad k, m \in \mathbb{N},
$$

for $b c=D i r$. Let us remind that $\tilde{q}(m)$ are the sine Fourier coefficients of the function $Q(x)$, i.e.,

$$
Q(x)=\sum_{m=1}^{\infty} \tilde{q}(m) \sqrt{2} \sin m x .
$$

The matrix representations of $K_{\lambda}\left(K_{\lambda} V K_{\lambda}\right) K_{\lambda}$ in (9.16) and (9.14) are the "building blocks" for the matrices of the products of the form $K_{\lambda}\left(K_{\lambda} V K_{\lambda}\right)^{s} K_{\lambda}$ that we have to estimate below. For convenience, we set

$$
V(m)=m w(m), \quad w \in \ell^{2}(2 \mathbb{Z}), \quad r(m)=\max (|w(m)|,|w(-m)|)
$$

if $b c=P e r^{ \pm}$, and

$$
\tilde{q}(0)=0, \quad r(m)=\tilde{q}(|m|), \quad m \in \mathbb{Z} .
$$

if $b c=D i r$. We use the notations (9.18) in the estimates related to $b c=P e r^{ \pm}$ below, and if one would use in a similar way (9.19) in the Dirichlet case, then the corresponding computations becomes practically identical (the only difference will be that in the Dirichlet case the summation will run over $\mathbb{Z}$ ).

Let us calculate the first term on the right-hand side of (9.13) (i.e., the term coming for $s=0$ ). We have

$$
\frac{1}{2 \pi i} \int_{C_{n}} \frac{V(k-m)}{\left(\lambda-k^{2}\right)\left(\lambda-m^{2}\right)} d \lambda= \begin{cases}\frac{V(k \mp n)}{\left(n^{2}-k^{2}\right)} & m= \pm n, \quad k \neq \pm n \\ \frac{V( \pm n-m)}{\left(n^{2}-m^{2}\right)} & k= \pm n, \quad m \neq \pm n \\ 0 & \text { otherwise. }\end{cases}
$$

Thus

$$
\begin{gathered}
A(n, 0)=\sum_{k, m}\left|\frac{1}{2 \pi i} \int_{C_{n}}\left\langle K_{\lambda}\left(K_{\lambda} V K_{\lambda}\right) K_{\lambda} e_{m}, e_{k}\right\rangle\right| \\
=\sum_{k \neq \pm n} \frac{|V(k-n)|}{\left|n^{2}-k^{2}\right|}+\sum_{k \neq \pm n} \frac{|V(k+n)|}{\left|n^{2}-k^{2}\right|}+\sum_{m \neq \pm n} \frac{|V(-n+m)|}{\left|n^{2}-m^{2}\right|}+\sum_{m \neq \pm n} \frac{|V(n-m)|}{\left|n^{2}-m^{2}\right|} .
\end{gathered}
$$

By the Cauchy inequality, we estimate the first sum on the right-hand side:

$$
\begin{aligned}
& \sum_{k \neq \pm n} \frac{|V(k-n)|}{\left|n^{2}-k^{2}\right|}=\sum_{k \neq \pm n} \frac{|k-n||w(k-n)|}{\left|n^{2}-k^{2}\right|} \\
& \leq \sum_{k \neq-n} \frac{r(k-n)}{|n+k|} \leq \sum_{k>0} \cdots+\sum_{k \leq 0, k \neq-n} \cdots
\end{aligned}
$$




$$
\begin{aligned}
\leq\left(\sum_{k>0} \frac{1}{|n+k|^{2}}\right)^{1 / 2} \cdot\|r\|+ & \left(\sum_{k \leq 0, k \neq-n} \frac{1}{|n+k|^{2}}\right)^{1 / 2}\left(\sum_{k \leq 0}(r(n-k))^{2}\right)^{1 / 2} \\
& \leq \frac{\|r\|}{\sqrt{n}}+\mathcal{E}_{n}(r) .
\end{aligned}
$$

Since each of the other three sums could be estimated in the same way, we get

$$
A(n, 0) \leq \sum_{k, m}\left|\frac{1}{2 \pi i} \int_{C_{n}}\left\langle K_{\lambda}\left(K_{\lambda} V K_{\lambda}\right) K_{\lambda} e_{m}, e_{k}\right\rangle d \lambda\right| \leq \frac{4\|r\|}{\sqrt{n}}+4 \mathcal{E}_{n}(r) .
$$

Next we estimate $A(n, s), s \geq 1$. By the matrix representation of $K_{\lambda}$ and $V-$ see $(9.16)$ - we have

$$
\left\langle K_{\lambda}\left(K_{\lambda} V K_{\lambda}\right)^{s+1} K_{\lambda} e_{m}, e_{k}\right\rangle=\frac{\Sigma(\lambda ; s, k, m)}{\left(\lambda-k^{2}\right)\left(\lambda-m^{2}\right)}
$$

where

$$
\Sigma(\lambda ; s, k, m)=\sum_{j_{1}, \ldots, j_{s}} \frac{V\left(k-j_{1}\right) V\left(j_{1}-j_{2}\right) \cdots V\left(j_{s-1}-j_{s}\right) V\left(j_{s}-m\right)}{\left(\lambda-j_{1}^{2}\right)\left(\lambda-j_{2}^{2}\right) \cdots\left(\lambda-j_{s}^{2}\right)},
$$

$k, m, j_{1}, \ldots, j_{s} \in n+2 \mathbb{Z}$. For convenience, we set also

$$
\Sigma(\lambda ; 0, k, m)=V(k-m) .
$$

In view of (9.15), we have

$$
A(n, s)=\sum_{k, m}\left|\frac{1}{2 \pi i} \int_{C_{n}} \frac{\Sigma(\lambda ; s, k, m)}{\left(\lambda-k^{2}\right)\left(\lambda-m^{2}\right)} d \lambda\right| .
$$

Let us consider the following sub-sums of $\operatorname{sum} \Sigma(\lambda ; s, k, m)$ defined in (9.24):

$$
\begin{gathered}
\Sigma^{0}(\lambda ; s, k, m)=\sum_{j_{1}, \ldots, j_{s} \neq \pm n} \cdots \quad \text { for } s \geq 1, \quad \Sigma^{0}(\lambda ; 0, k, m):=V(k-m) ; \\
\Sigma^{1}(\lambda ; s, k, m)=\sum_{\exists \text { one } j_{\nu}= \pm n} \cdots \quad \text { for } s \geq 1 ; \\
\Sigma^{*}(\lambda ; s, k, m)=\sum_{\exists j_{\nu}= \pm n} \ldots, \quad \Sigma^{* *}(\lambda ; s, k, m)=\sum_{\exists j_{\nu}, j_{\mu}= \pm n} \ldots, \quad s \geq 2
\end{gathered}
$$

(i.e., $\Sigma^{0}$ is the sub-sum of the sum $\Sigma$ in (9.24) over those indices $j_{1}, \ldots, j_{s}$ that are different from $\pm n$, in $\Sigma^{1}$ exactly one summation index is equal to $\pm n$, in $\Sigma^{*}$ at least one summation index is equal to $\pm n$, and in $\Sigma^{* *}$ at least two summation indices are equal to $\pm n)$. Notice that

$$
\Sigma(\lambda ; s, k, m)=\Sigma^{0}(\lambda ; s, k, m)+\Sigma^{*}(\lambda ; s, k, m), \quad s \geq 1,
$$

and

$$
\Sigma(\lambda ; s, k, m)=\Sigma^{0}(\lambda ; s, k, m)+\Sigma^{1}(\lambda ; s, k, m)+\Sigma^{* *}(\lambda ; s, k, m), \quad s \geq 2 .
$$

In these notations we have

$$
\sum_{m, k \neq \pm n}\left|\frac{1}{2 \pi i} \int_{C_{n}} \frac{\Sigma^{0}(\lambda ; s, k, m)}{\left(\lambda-k^{2}\right)\left(\lambda-m^{2}\right)} d \lambda\right|=0
$$


because, for $m, k \neq \pm n$, the integrand is an analytic function of $\lambda$ in the disc $\left\{\lambda:\left|\lambda-n^{2}\right| \leq n / 4\right\}$.

Therefore, $A(n, s)$ could be estimated as follows:

$$
A(n, 1) \leq \sum_{i=1}^{5} A_{i}(n, 1)
$$

and

$$
A(n, s) \leq \sum_{i=1}^{7} A_{i}(n, s), \quad s \geq 2
$$

where

$$
\begin{gathered}
A_{1}(n, s)=\sum_{k, m= \pm n} n \cdot \sup _{\lambda \in C_{n}}\left|\frac{\Sigma(\lambda ; s, k, m)}{\left(\lambda-k^{2}\right)\left(\lambda-m^{2}\right)}\right|, \\
A_{2}(n, s)=\sum_{k= \pm n, m \neq \pm n} n \cdot \sup _{\lambda \in C_{n}}\left|\frac{\Sigma^{0}(\lambda ; s, k, m)}{\left(\lambda-k^{2}\right)\left(\lambda-m^{2}\right)}\right| \\
A_{3}(n, s)=\sum_{k= \pm n, m \neq \pm n} n \cdot \sup _{\lambda \in C_{n}}\left|\frac{\Sigma^{*}(\lambda ; s, k, m)}{\left(\lambda-k^{2}\right)\left(\lambda-m^{2}\right)}\right|, \\
A_{4}(n, s)=\sum_{k \neq \pm n, m= \pm n} n \cdot \sup _{\lambda \in C_{n}}\left|\frac{\Sigma^{0}(\lambda ; s, k, m)}{\left(\lambda-k^{2}\right)\left(\lambda-m^{2}\right)}\right|, \\
A_{5}(n, s)=\sum_{k \neq \pm n, m= \pm n} n \cdot \sup _{\lambda \in C_{n}}\left|\frac{\Sigma^{*}(\lambda ; s, k, m)}{\left(\lambda-k^{2}\right)\left(\lambda-m^{2}\right)}\right| \\
A_{6}(n, s)=\sum_{k, m \neq \pm n} n \cdot \sup _{\lambda \in C_{n}}\left|\frac{\Sigma^{1}(\lambda ; s, k, m)}{\left(\lambda-k^{2}\right)\left(\lambda-m^{2}\right)}\right| \\
A_{7}(n, s)=\sum_{k, m \neq \pm n} n \cdot \sup _{\lambda \in C_{n}}\left|\frac{\Sigma^{* *}(\lambda ; s, k, m)}{\left(\lambda-k^{2}\right)\left(\lambda-m^{2}\right)}\right| .
\end{gathered}
$$

First we estimate $A_{1}(n, s)$. By (9.16) and [16], Lemma 19 (inequalities (5.30),(5.31)), (9.40) $\sup _{\lambda \in C_{n}}\left\|K_{\lambda}\right\|=\frac{2}{\sqrt{n}}, \quad \sup _{\lambda \in C_{n}}\left\|K_{\lambda} V K_{\lambda}\right\| \leq \rho_{n}:=C\left(\frac{\|r\|}{\sqrt{n}}+\mathcal{E}_{\sqrt{n}}(r)\right)$, where $r=(r(m))$ is defined by the relations (9.18) and $C$ is an absolute constant.

LEMMA 46. In the above notations

$$
\sup _{\lambda \in C_{n}}\left|\frac{\Sigma(\lambda ; s, k, m)}{\left(\lambda-k^{2}\right)\left(\lambda-m^{2}\right)}\right| \leq \frac{1}{n} \rho_{n}^{s+1} .
$$

Proof. Indeed, in view of (9.24) and (9.40), we have

$$
\begin{gathered}
\left|\frac{\Sigma(\lambda ; s, k, m)}{\left(\lambda-k^{2}\right)\left(\lambda-m^{2}\right)}\right|=\left|K_{\lambda}\left(K_{\lambda} V K_{\lambda}\right)^{s+1} K_{\lambda} e_{k}, e_{m}\right\rangle \mid \\
\leq\left\|K_{\lambda}\left(K_{\lambda} V K_{\lambda}\right)^{s+1} K_{\lambda}\right\| \leq\left\|K_{\lambda}\right\| \cdot\left\|K_{\lambda} V K_{\lambda}\right\|^{s+1} \cdot\left\|K_{\lambda}\right\| \leq \frac{1}{n} \rho_{n}^{s+1},
\end{gathered}
$$

which proves (9.41). 
Now we estimate $A_{1}(n, s)$. By $(9.41)$,

$$
A_{1}(n, s)=\sum_{m, k= \pm n} n \cdot \sup _{\lambda \in C_{n}}\left|\frac{\Sigma(\lambda ; s, k, m)}{\left(\lambda-k^{2}\right)\left(\lambda-m^{2}\right)}\right| \leq 4 \rho_{n}^{s+1} .
$$

To estimate $A_{2}(n, s)$, we consider $\Sigma^{0}(\lambda ; s, k, m)$ for $k= \pm n$. From the elementary inequality

$$
\frac{1}{\left|\lambda-j^{2}\right|} \leq \frac{2}{\left|n^{2}-j^{2}\right|} \quad \text { for } \quad \lambda \in C_{n}, j \in n+2 \mathbb{Z}, j \neq \pm n
$$

it follows, for $m \neq \pm n$,

$$
\begin{gathered}
\sup _{\lambda \in C_{n}}\left|\frac{\Sigma^{0}(\lambda ; s, \pm n, m)}{\left(\lambda-n^{2}\right)\left(\lambda-m^{2}\right)}\right| \leq \frac{1}{n} \cdot 2^{s+1} \times \\
\times \sum_{j_{1}, \ldots, j_{s} \neq \pm n} \frac{\left|V\left( \pm n-j_{1}\right) V\left(j_{1}-j_{2}\right) \cdots V\left(j_{s-1}-j_{s}\right) V\left(j_{s}-m\right)\right|}{\left|n^{2}-j_{1}^{2}\right|\left|n^{2}-j_{2}^{2}\right| \cdots\left|n^{2}-j_{s}^{2}\right|\left|n^{2}-m^{2}\right|} .
\end{gathered}
$$

Thus, taking the sum of both sides of (9.44) over $m \neq \pm n$, we get

$$
A_{2}(n, s) \leq 2^{s+1}[L(s+1, n)+L(s+1,-n)]
$$

where

$$
L(p, d):=\sum_{i_{1}, \ldots, i_{p} \neq \pm n} \frac{\left|V\left(d-i_{1}\right)\right|}{\left|n^{2}-i_{1}^{2}\right|} \cdot \frac{\left|V\left(i_{1}-i_{2}\right)\right|}{\left|n^{2}-i_{2}^{2}\right|} \cdots \frac{\left|V\left(i_{p-1}-i_{p}\right)\right|}{\left|n^{2}-i_{p}^{2}\right|} .
$$

The roles of $k$ and $m$ in $A_{2}(n, s)$ and $A_{4}(n, s)$ are symmetric, so $A_{4}(n, s)$ could be estimated in an analogous way. Indeed, for $k \neq \pm n$, we have

$$
\begin{gathered}
\sup _{\lambda \in C_{n}}\left|\frac{\Sigma^{0}(\lambda ; s, k, \pm n)}{\left(\lambda-k^{2}\right)\left(\lambda-n^{2}\right)}\right| \leq \frac{1}{n} \cdot 2^{s+1} \times \\
\times \sum_{j_{1}, \ldots, j_{s} \neq \pm n} \frac{\left|V\left(k-j_{1}\right) V\left(j_{1}-j_{2}\right) \cdots V\left(j_{s-1}-j_{s}\right) V\left(j_{s}- \pm n\right)\right|}{\left|n^{2}-k^{2}\right|\left|n^{2}-j_{1}^{2}\right|\left|n^{2}-j_{2}^{2}\right| \cdots\left|n^{2}-j_{s}^{2}\right|} .
\end{gathered}
$$

Thus, taking the sum of both sides of (9.47) over $k \neq \pm n$, we get

$$
A_{4}(n, s) \leq 2^{s+1}[R(s+1, n)+R(s+1,-n)],
$$

where

$$
R(p, d):=\sum_{i_{1}, \ldots, i_{p} \neq \pm n} \frac{\left|V\left(i_{1}-i_{2}\right)\right|}{\left|n^{2}-i_{1}^{2}\right|} \cdots \frac{\left|V\left(i_{p-1}-i_{p}\right)\right|}{\left|n^{2}-i_{p-1}^{2}\right|} \cdot \frac{\left|V\left(i_{p}-d\right)\right|}{\left|n^{2}-i_{p}^{2}\right|} .
$$

Below (see Lemma 47 and its proof in Subsection 9.3) we estimate the sums $L(p, \pm n)$ and $R(p, \pm n)$. But now we are going to show that $A_{i}(n, s), i=3,5,6,7$, could be estimated in terms of $L$ and $R$ from (9.46), (9.49) as well.

To estimate $A_{6}(n, s)$ we write the expression $\frac{\Sigma^{1}(\lambda ; s, k, m)}{\left(\lambda-k^{2}\right)\left(\lambda-m^{2}\right)}$ in the form

$$
\sum_{\nu=1}^{s} \sum_{d= \pm n} \frac{1}{\lambda-k^{2}} \Sigma^{0}(\lambda ; \nu-1, k, d) \frac{1}{\lambda-n^{2}} \Sigma^{0}(\lambda ; s-\nu, d, m) \frac{1}{\lambda-m^{2}}
$$

By (9.43), the absolute values of the terms of this double sum do not exceed: 
(a) for $\nu=1$

$2^{s+1} \cdot \frac{|V(k- \pm n)|}{\left|n^{2}-k^{2}\right|} \cdot \frac{1}{n} \cdot \sum_{i_{1}, \ldots, i_{s-1} \neq \pm n} \frac{\left|V\left( \pm n-i_{1}\right)\right|\left|V\left(i_{1}-i_{2}\right)\right| \cdots\left|V\left(i_{s-1}-m\right)\right|}{\left|n^{2}-i_{1}^{2}\right| \cdots\left|n^{2}-i_{s-1}^{2}\right|\left|n^{2}-m^{2}\right|}$.

(b) for $\nu=s$

$2^{s+1} \cdot\left(\sum_{i_{1}, \ldots, i_{s-1} \neq \pm n} \frac{\left|V\left(k-i_{1}\right)\right|\left|V\left(i_{1}-i_{2}\right)\right| \cdots\left|V\left(i_{s-1}- \pm n\right)\right|}{\left|n^{2}-k^{2}\right|\left|n^{2}-i_{1}^{2}\right|\left|n^{2}-i_{2}^{2}\right| \cdots\left|n^{2}-i_{s-1}^{2}\right|}\right) \cdot \frac{1}{n} \cdot \frac{|V( \pm n-m)|}{\left|n^{2}-m^{2}\right|}$

(c) for $1<\nu<s$

$$
\begin{aligned}
2^{s+1} & \left.\cdot \sum_{i_{1}, \ldots, i_{\nu-1} \neq \pm n} \frac{\left|V\left(k-i_{1}\right)\right|\left|V\left(i_{1}-i_{2}\right)\right| \cdots\left|V\left(i_{\nu-1}- \pm n\right)\right|}{\left|n^{2}-k^{2}\right|\left|n^{2}-i_{1}^{2}\right|\left|n^{2}-i_{2}^{2}\right| \cdots\left|n^{2}-i_{\nu-1}^{2}\right|}\right) \cdot \frac{1}{n} \\
& \times \sum_{i_{1}, \ldots, i_{s-\nu} \neq \pm n} \frac{\left|V\left( \pm n-i_{1}\right)\right|\left|V\left(i_{1}-i_{2}\right)\right| \cdots\left|V\left(i_{s-\nu}-m\right)\right|}{\left|n^{2}-i_{1}^{2}\right| \cdots\left|n^{2}-i_{s-\nu}^{2}\right|\left|n^{2}-m^{2}\right|} .
\end{aligned}
$$

Therefore, taking the sum over $m, k \neq \pm n$, we get

$$
A_{6}(n, s) \leq 2^{s+1} \cdot \sum_{\nu=1}^{s} \sum_{d= \pm n} R(\nu, d) \cdot L(s+1-\nu, d) .
$$

One could estimate $A_{3}(n, s), A_{5}(n, s)$ and $A_{7}(n, s)$ in an analogous way. We will write the core formulas but omit some details.

To estimate $A_{3}(n, s)$, we use the identity

$$
\frac{\Sigma(\lambda ; s, k, \pm n)}{\left(\lambda-k^{2}\right)\left(\lambda-n^{2}\right)}=\sum_{\nu=1}^{s} \sum_{d= \pm n} \frac{1}{\lambda-k^{2}} \Sigma^{0}(\lambda ; \nu-1, k, d) \frac{1}{\lambda-n^{2}} \frac{\Sigma(\lambda ; s-\nu, d, \pm n)}{\lambda-n^{2}} .
$$

In view of (9.41), (9.43) and (9.49), from here it follows that

$$
A_{3}(n, s) \leq 2^{s+1} \cdot \sum_{\nu=1}^{s} \sum_{d= \pm n} R(\nu, d) \cdot \rho_{n}^{s-\nu+1} .
$$

We estimate $A_{5}(n, s)$ by using the identity

$$
\frac{\Sigma(\lambda ; s, \pm n, m)}{\left(\lambda-n^{2}\right)\left(\lambda-m^{2}\right)}=\sum_{\nu=1}^{s} \sum_{d= \pm n} \frac{1}{\lambda-n^{2}} \Sigma(\lambda ; \nu-1, \pm n, d) \frac{1}{\lambda-n^{2}} \frac{\Sigma^{0}(\lambda ; s-\nu, d, m)}{\lambda-m^{2}} .
$$

In view of (9.41), (9.43) and (9.46), from here it follows that

$$
A_{5}(n, s) \leq 2^{s+1} \cdot \sum_{\nu=1}^{s} \sum_{d= \pm n} \rho_{n}^{\nu} \cdot L(s-\nu+1, d) .
$$

Finally, to estimate $A_{7}(n, s)$ we use the identity

$$
\begin{aligned}
& \frac{\Sigma(\lambda ; s, k, m)}{\left(\lambda-k^{2}\right)\left(\lambda-m^{2}\right)}=\sum_{1 \leq \nu<\mu \leq s}^{s} \sum_{d_{1}, d_{2}= \pm n} \frac{1}{\lambda-k^{2}} \Sigma^{0}\left(\lambda ; \nu-1, k, d_{1}\right) \times \\
& \times \frac{1}{\lambda-n^{2}} \Sigma\left(\lambda ; \mu-\nu-1, d_{1}, d_{2}\right) \frac{1}{\lambda-n^{2}} \Sigma^{0}\left(\lambda ; s-\mu, d_{2}, m\right) \frac{1}{\lambda-m^{2}}
\end{aligned}
$$


In view of (9.41), (9.43), (9.46) and (9.49), from here it follows that

$$
A_{7}(n, s) \leq 2^{s} \cdot \sum_{1 \leq \nu<\mu \leq s} \sum_{d_{1}, d_{2}= \pm n} R\left(\nu, d_{1}\right) \cdot \rho_{n}^{\mu-\nu} \cdot L\left(s-\mu+1, d_{2}\right) .
$$

Next we estimate $L(p, \pm n)$ and $R(p, \pm n)$. Changing the indices in (9.49) by

$$
j_{\nu}=-i_{p+1-\nu}, \quad 1 \leq \nu \leq p
$$

we get

$$
R(p, d)=L(p,-d) .
$$

Therefore, it is enough to estimate only $L(p, \pm n)$.

LEMMA 47. In the above notations, there exists a sequence of positive numbers $\varepsilon_{n} \rightarrow 0$ such that, for large enough $n$,

$$
L(s, \pm n) \leq\left(\varepsilon_{n}\right)^{s}, \quad \forall s \in \mathbb{N} .
$$

The proof of this lemma is technical. It is given in detail in Subsection 9.3. Then in Subsection 9.4 we complete the proof of Theorem 45. With (9.54) and (9.55), in Subsection 9.4 we will use Lemma 47 in the following form.

COROLlaRY 48. In the above notations, there exists a sequence of positive numbers $\varepsilon_{n} \rightarrow 0$ such that, for large enough $n$,

$$
\max \{L(s, \pm n), R(s, \pm n)\} \leq\left(\varepsilon_{n}\right)^{s}, \quad \forall s \in \mathbb{N} .
$$

9.3. Technical inequalities and their proofs. We follow the notations from Subsection 9.2. Now we prove Lemma 47.

Proof. First we show that

$$
L(s, \pm n) \leq \sigma(n, s), \quad s \geq 1
$$

where

$$
\sigma(n, 1)=\sum_{j_{1} \neq \pm n} \frac{r\left(n+j_{1}\right)}{\left|n^{2}-j_{1}^{2}\right|}
$$

for $s \geq 2$

$$
\begin{aligned}
\sigma(n, s):=\sum_{j_{1}, \ldots, j_{s} \neq \pm n} & \left(\frac{1}{\left|n-j_{1}\right|}+\frac{1}{\left|n+j_{2}\right|}\right) \cdots\left(\frac{1}{\left|n-j_{s-1}\right|}+\frac{1}{\left|n+j_{s}\right|}\right) \frac{1}{\left|n-j_{s}\right|} \\
& \times r\left(n+j_{1}\right) r\left(j_{1}+j_{2}\right) \cdots r\left(j_{s-1}+j_{s}\right),
\end{aligned}
$$

and the sequence $r=(r(m))$ is defined by (9.18).

For $s=1$ we have, with $i_{1}=-j_{1}$,

$$
\begin{gathered}
L(1, n)=\sum_{j_{1} \neq \pm n} \frac{\left|V\left(n-j_{1}\right)\right|}{\left|n^{2}-j_{1}^{2}\right|}=\sum_{i_{1} \neq \pm n} \frac{\left|V\left(n+i_{1}\right)\right|}{\left|n^{2}-i_{1}^{2}\right|} \\
=\sum_{i_{1} \neq \pm n} \frac{\left|w\left(n+i_{1}\right)\right|}{\left|n-i_{1}\right|} \leq \sum_{i_{1} \neq \pm n} \frac{r\left(n+i_{1}\right)}{\left|n-i_{1}\right|}
\end{gathered}
$$

(where (9.18) is used). In an analogous way we get

$$
L(1,-n)=\sum_{j_{1} \neq \pm n} \frac{\left|V\left(-n-j_{1}\right)\right|}{\left|n^{2}-j_{1}^{2}\right|}=\sum_{i_{1} \neq \pm n} \frac{\left|w\left(-n-i_{1}\right)\right|}{\left|n-i_{1}\right|} \leq \sum_{i_{1} \neq \pm n} \frac{r\left(n+i_{1}\right)}{\left|n-i_{1}\right|}
$$


so, (9.57) holds for $s=1$.

Let $s \geq 2$. Changing the indices of summation in (9.46) (considered with $p=s$ and $d$ ) by $j_{\nu}=(-1)^{\nu} i_{\nu}$, we get

$$
\begin{aligned}
& L(s, n)=\sum_{j_{1}, \ldots, j_{s} \neq \pm n} \frac{\left|V\left(n+j_{1}\right)\right|}{\left|n^{2}-j_{1}^{2}\right|} \frac{\left|V\left(-j_{1}-j_{2}\right)\right|}{\left|n^{2}-j_{1}^{2}\right|} \cdots \frac{\left|V\left[(-1)^{s-1}\left(j_{s-1}+j_{s}\right)\right]\right|}{\left|j_{s}^{2}-n^{2}\right|} \\
= & \sum_{j_{1}, \ldots, j_{s} \neq \pm n} \frac{\left|n+j_{1}\right|\left|j_{2}+j_{1}\right| \cdots\left|j_{s}+j_{s-1}\right|}{\left|j_{1}^{2}-n^{2}\right|\left|j_{2}^{2}-n^{2}\right| \cdots\left|j_{s}^{2}-n^{2}\right|}\left|w\left(n+j_{1}\right) \cdots w\left[(-1)^{s-1}\left(j_{s-1}+j_{s}\right)\right]\right| \\
\leq & \sum_{j_{1}, \ldots, j_{s} \neq \pm n} \frac{\left|j_{2}+j_{1}\right| \cdots\left|j_{s}+j_{s-1}\right|}{\left|n-j_{1}\right|\left|n^{2}-j_{2}^{2}\right| \cdots\left|n^{2}-j_{s}^{2}\right|} r\left(n+j_{1}\right) r\left(j_{1}+j_{2}\right) \cdots r\left(j_{s-1}+j_{s}\right) .
\end{aligned}
$$

By the identity

$$
\frac{i+k}{(n-i)(n+k)}=\frac{1}{n-i}-\frac{1}{n+k},
$$

we get that the latter sum does not exceed

$$
\begin{aligned}
& \sum_{j_{1}, \ldots, j_{s} \neq \pm n}\left|\frac{1}{n-j_{1}}-\frac{1}{n+j_{2}}\right| \cdots\left|\frac{1}{n-j_{s-1}}-\frac{1}{n+j_{s}}\right| \frac{1}{\left|n-j_{s}\right|} \\
& \quad \times r\left(n+j_{1}\right) r\left(j_{1}+j_{2}\right) \cdots r\left(j_{s-1}+j_{s}\right) \leq \sigma(n, s) .
\end{aligned}
$$

Changing the indices of summation in (9.46) (considered with $p=s$ and $d=$ $-n)$ by $j_{\nu}=(-1)^{\nu+1} i_{\nu}$, one can show that $L(s,-n) \leq \sigma(n, s)$. Since the proof is the same we omit the details. This completes the proof of (9.57).

In view of (9.57), Lemma 47 will be proved if we show that there exists a sequence of positive numbers $\varepsilon_{n} \rightarrow 0$ such that, for large enough $n$,

$$
\sigma(n, s) \leq\left(\varepsilon_{n}\right)^{s}, \quad \forall s \in \mathbb{N} .
$$

In order to prove (9.60) we need the following statements.

LeMma 49. Let $r=(r(k)) \in \ell^{2}(2 \mathbb{Z}), r(k) \geq 0$, and let

$$
\sigma_{1}(n, s ; m)=\sum_{j_{1}, \ldots, j_{s} \neq n} \frac{r\left(m+j_{1}\right)}{\left|n-j_{1}\right|} \frac{r\left(j_{1}+j_{2}\right)}{\left|n-j_{2}\right|} \cdots \frac{r\left(j_{s-1}+j_{s}\right)}{\left|n-j_{s}\right|}, \quad n, s \in \mathbb{N},
$$

where $m, j_{1}, \ldots, j_{s} \in n+2 \mathbb{Z}$. Then, with

$$
\tilde{\rho}_{n}:=\mathcal{E}_{n}(r)+2\|r\| / \sqrt{n},
$$

we have, for $n \geq 4$,

$$
\begin{gathered}
\sigma_{1}(n, 1 ; m) \leq \begin{cases}\tilde{\rho}_{n} & \text { if }|m-n| \leq n / 2, \\
\|r\| & \text { for arbitrary } m \in n+2 \mathbb{Z},\end{cases} \\
\sigma_{1}(n, 2 p ; m) \leq\left(2\|r\| \tilde{\rho}_{n}\right)^{p}, \quad \sigma_{1}(n, 2 p+1 ; m) \leq\|r\| \cdot\left(2\|r\| \tilde{\rho}_{n}\right)^{p} .
\end{gathered}
$$

Proof. Let us recall that

$$
\sum_{k=1}^{\infty} \frac{1}{k^{2}}=\pi^{2} / 6, \quad \sum_{k+1}^{\infty} \frac{1}{k^{2}}<\sum_{k+1}^{\infty}\left(\frac{1}{k-1}-\frac{1}{k}\right)=\frac{1}{n} .
$$

Therefore, one can easily see that

$$
\sum_{i \in 2 \mathbb{Z}, i \neq 0} \frac{1}{i^{2}}=\pi^{2} / 12<1, \quad \sum_{i \in 2 \mathbb{Z},|i|>n / 2} \frac{1}{i^{2}}<4 / n, \quad n \geq 4 .
$$


By the Cauchy inequality,

$$
\sigma_{1}(n, 1 ; m)=\sum_{j_{1} \neq n} \frac{r\left(m+j_{1}\right)}{\left|n-j_{1}\right|} \leq\left(\sum_{j_{1} \neq \pm n}\left|n-j_{1}\right|^{-2}\right)^{1 / 2} \cdot\|r\| \leq\|r\|,
$$

which proves the second case in (9.63).

If $|m-n| \leq n / 2$ then we have $n / 2 \leq m \leq 3 n / 2$. Let us write $\sigma_{1}(n, 1 ; m)$ in the form

$$
\sigma_{1}(n, 1 ; m)=\sum_{0<\left|j_{1}-n\right| \leq n / 2} \frac{r\left(m+j_{1}\right)}{\left|n-j_{1}\right|}+\sum_{\left|j_{1}-n\right|>n / 2} \frac{r\left(m+j_{1}\right)}{\left|n-j_{1}\right|}
$$

and apply the Cauchy inequality to each of the above sums. In the first sum $n / 2 \leq j \leq 3 n / 2$, so $j+m \geq n$, and therefore, we get

$$
\sigma_{1}(n, 1 ; m) \leq\left(\sum_{i \geq n}|r(i)|^{2}\right)^{1 / 2} \cdot 1+\|r\| \cdot\left(\sum_{\left|n-j_{1}\right|>n / 2}\left|j_{1}-n\right|^{-2}\right)^{1 / 2} .
$$

Thus

$$
\sigma_{1}(n, 1 ; m) \leq \mathcal{E}_{n}(r)+\frac{2\|r\|}{\sqrt{n}}=\tilde{\rho}_{n} \quad \text { if } \quad|n-m| \leq n / 2 .
$$

This completes the proof of (9.63).

Next we estimate $\sigma_{1}(n, 2 ; m)$. We have

$$
\begin{gathered}
\sigma_{1}(n, 2 ; m)=\sum_{j_{1} \neq n} \frac{r\left(m+j_{1}\right)}{\left|n-j_{1}\right|} \sum_{j_{2} \neq n} \frac{r\left(j_{1}+j_{2}\right)}{\left|n-j_{2}\right|} \\
=\sum_{0<\left|j_{1}-n\right| \leq n / 2} \frac{r\left(m+j_{1}\right)}{\left|n-j_{1}\right|} \cdot \sigma_{1}\left(n, 1 ; j_{1}\right)+\sum_{\left|j_{1}-n\right|>n / 2} \frac{r\left(m+j_{1}\right)}{\left|n-j_{1}\right|} \cdot \sigma_{1}\left(n, 1 ; j_{1}\right)
\end{gathered}
$$

By the Cauchy inequality and (9.63), we get

$$
\sum_{0<\left|j_{1}-n\right| \leq n / 2} \frac{r\left(m+j_{1}\right)}{\left|n-j_{1}\right|} \sigma_{1}\left(n, 1 ; j_{1}\right) \leq\|r\| \cdot \sup _{0<\left|j_{1}-n\right| \leq n / 2} \sigma_{1}\left(n, 1 ; j_{1}\right) \leq\|r\| \tilde{\rho}_{n},
$$

and

$$
\sum_{\left|j_{1}-n\right|>n / 2} \frac{r\left(m+j_{1}\right)}{\left|n-j_{1}\right|} \sigma_{1}\left(n, 1 ; j_{1}\right) \leq \sum_{\left|j_{1}-n\right|>n / 2} \frac{r\left(m+j_{1}\right)}{\left|n-j_{1}\right|} \cdot\|r\| \leq \frac{2\|r\|}{\sqrt{n}} \cdot\|r\| .
$$

Thus, in view of (9.62), we have

$$
\sigma_{1}(n, 2 ; m) \leq 2\|r\| \cdot \tilde{\rho}_{n} .
$$

On the other hand, for every $s \in \mathbb{N}$, we have

$$
\begin{gathered}
\sigma_{1}(n, s+2 ; m)=\sum_{j_{1}, \ldots, j_{s} \neq n} \frac{r\left(m+j_{1}\right)}{\left|n-j_{1}\right|} \cdots \frac{r\left(j_{s-1}+j_{s}\right)}{\left|n-j_{s}\right|} \\
\times \sum_{j_{s+1}, j_{s+2} \neq n} \frac{r\left(j_{s}+j_{s+1}\right)}{\left|n-j_{s+1}\right|} \frac{r\left(j_{s+1}+j_{s+2}\right)}{\left|n-j_{s+2}\right|}=\sigma_{1}(n, s ; m) \cdot \sup _{j_{s}} \sigma_{1}\left(n, 2 ; j_{s}\right) .
\end{gathered}
$$

Thus, by (9.65),

$$
\sigma_{1}(n, s+2 ; m) \leq \sigma_{1}(n, s ; m) \cdot 2\|r\| \tilde{\rho}_{n} .
$$


Now it is easy to see, by induction in $p$, that (9.63), (9.65) and (9.66) imply (9.64).

Lemma 50. Let $r=(r(k)) \in \ell^{2}(2 \mathbb{Z})$ be the sequence defined by (9.18), and let

$$
\sigma_{2}(n, s ; m)=\sum_{j_{1}, \ldots, j_{s} \neq n} r\left(m+j_{1}\right) \frac{r\left(j_{1}+j_{2}\right)}{\left|n+j_{2}\right|} \cdots \frac{r\left(j_{s-2}+j_{s-1}\right)}{\left|n+j_{s-1}\right|} \frac{r\left(j_{s-1}+j_{s}\right)}{\left|n^{2}-j_{s}^{2}\right|}
$$

where $n \in \mathbb{N}, s \geq 2$ and $m, j_{1}, \ldots, j_{s} \in n+2 \mathbb{Z}$. Then we have

$$
\sigma_{2}(n, 2 ; m) \leq\|r\|^{2} \cdot \frac{2 \log 6 n}{n}
$$

and

$$
\sigma_{2}(n, s ; m) \leq\|r\|^{2} \cdot \frac{2 \log 6 n}{n} \cdot \sup _{k} \sigma_{1}(n, s-2 ; k), \quad s \geq 3 .
$$

Proof. We have

$$
\sigma_{2}(n, 2, m)=\sum_{j_{2} \neq \pm n} \frac{1}{\left|n^{2}-j_{2}^{2}\right|} \sum_{j_{1} \neq \pm n} r\left(m+j_{1}\right) r\left(j_{1}+j_{2}\right) .
$$

By the Cauchy inequality, the sum over $j_{1} \neq \pm n$ does not exceed $\|r\|^{2}$. Let us notice that

$$
\sum_{j \neq \pm n} \frac{1}{n^{2}-j^{2}}=\frac{2}{n} \sum_{1}^{2 n} \frac{1}{k}-\frac{1}{2 n^{2}}<\frac{2 \log 6 n}{n} .
$$

Therefore, (9.70) and (9.71) imply (9.68).

If $s \geq 3$ then the sum $\sigma_{2}(n, s ; m)$ can be written as a product of three sums:

$$
\sum_{j_{s} \neq \pm n} \frac{1}{\left|n^{2}-j_{s}^{2}\right|} \sum_{j_{2}, \ldots, j_{s-1} \neq \pm n} \frac{r\left(j_{2}+j_{3}\right)}{\left|n+j_{2}\right|} \cdots \frac{r\left(j_{s-1}+j_{s}\right)}{\left|n+j_{s-1}\right|} \sum_{j_{1} \neq \pm n} r\left(m+j_{1}\right) r\left(j_{1}+j_{2}\right) .
$$

Changing the sign of all indices, one can easily see that the middle sum (over $\left.j_{2}, \ldots, j_{s-1}\right)$ equals $\sigma_{1}\left(n ; s-2, j_{s}\right)$. Thus, we have

$$
\sigma_{2}(n, s ; m) \leq \sum_{j_{s} \neq \pm n} \frac{1}{\left|n^{2}-j_{s}^{2}\right|} \sigma_{1}\left(n ; s-2, j_{s}\right) \cdot \sup _{j_{2}} \sum_{j_{1} \neq \pm n} r\left(m+j_{1}\right) r\left(j_{1}+j_{2}\right) .
$$

By the Cauchy inequality, the sum over $j_{1} \neq \pm n$ does not exceed $\|r\|^{2}$.

Therefore, by (9.71), we get (9.69).

Proof of Lemma 47. We set

$$
\varepsilon_{n}=M \cdot\left[\left(\frac{2 \log 6 n}{n}\right)^{1 / 4}+\left(\tilde{\rho}_{n}\right)^{1 / 2}\right],
$$

where $M=4(1+\|r\|)$ is chosen so that for large enough $n$

$$
\sup _{m} \sigma_{1}(n, 2 p, m) \leq\left(\varepsilon_{n} / 2\right)^{2 p}, \quad \sup _{m} \sigma_{1}(n, 2 p+1, m) \leq\|r\|\left(\varepsilon_{n} / 2\right)^{2 p} .
$$

Then, for large enough $n$, we have

$$
\sup _{m} \sigma_{2}(n, s, m) \leq \frac{1}{M}\left(\varepsilon_{n} / 2\right)^{s+1} .
$$


Indeed, by the choice of $M$, we have

$$
\|r\|^{2} \cdot \frac{2 \log 6 n}{n} \leq\|r\|^{2}\left(\varepsilon_{n} / M\right)^{4} \leq \frac{1}{M^{2}}\left(\varepsilon_{n} / 2\right)^{4} .
$$

Since $\varepsilon_{n} \rightarrow 0$, there is $n_{0}$ such that $\varepsilon_{n}<1$ for $n \geq n_{0}$. Therefore, if $n \geq n_{0}$, then (9.68) and (9.75) yields (9.74) for $s=2$. If $s=2 p$ with $p>1$, then (9.69), (9.75) and (9.73) imply, for $n \geq n_{0}$,

$$
\sup _{m} \sigma_{2}(n, 2 p, m) \leq \frac{1}{M^{2}}\left(\varepsilon_{n} / 2\right)^{4} \cdot\left(\varepsilon_{n} / 2\right)^{2 p-2} \leq \frac{1}{M}\left(\varepsilon_{n} / 2\right)^{2 p+1} .
$$

In an analogous way, for $n \geq n_{0}$, we get

$$
\sup _{m} \sigma_{2}(n, 2 p+1, m) \leq \frac{1}{M^{2}}\left(\varepsilon_{n} / 2\right)^{4} \cdot\|r\|\left(\varepsilon_{n} / 2\right)^{2(p-1)} \leq \frac{1}{M}\left(\varepsilon_{n} / 2\right)^{2 p+2},
$$

which completes the proof of (9.74).

Next we estimate $\sigma(n, s)$ by induction in $s$. By (9.63), we have for $n \geq n_{0}$,

$$
\sigma(n, 1)=\sum_{j_{1} \neq \pm n} \frac{r\left(n-j_{1}\right)}{\left|n-j_{1}\right|}=\sigma_{1}(n, 1 ; n) \leq \tilde{\rho}_{n} \leq\left(\varepsilon_{n} / 2\right)^{2} \leq \varepsilon_{n}
$$

For $s=2$ we get, in view of (9.73) and (9.73):

$$
\begin{gathered}
\sigma(n, 2)=\sum_{j_{1}, j_{2} \neq \pm n}\left(\frac{1}{\left|n-j_{1}\right|}+\frac{1}{\left|n+j_{2}\right|}\right) \frac{1}{\left|n-j_{2}\right|} r\left(n+j_{1}\right) r\left(j_{1}+j_{2}\right) \\
\leq \sum_{j_{1}, j_{2} \neq \pm n} \frac{r\left(n+j_{1}\right) r\left(j_{1}+j_{2}\right)}{\left|n-j_{1}\right|\left|n-j_{2}\right|}+\sum_{j_{1}, j_{2} \neq \pm n} \frac{1}{\left|n+j_{2}\right|} \cdot \frac{1}{\left|n-j_{2}\right|} r\left(n+j_{1}\right) r\left(j_{1}+j_{2}\right) \\
=\sigma_{1}(n, 2, n)+\sigma_{2}(n, 2, n) \leq\left(\varepsilon_{n} / 2\right)^{2}+\left(\varepsilon_{n} / 2\right)^{2} \leq\left(\varepsilon_{n}\right)^{2} .
\end{gathered}
$$

Next we estimate $\sigma(n, s), s \geq 2$, Recall that $\sigma(n, s)$ is the sum of terms of the form

$$
\Pi\left(j_{1}, \ldots, j_{s}\right) r\left(n+j_{1}\right) r\left(j_{1}+j_{2}\right) \cdots r\left(j_{s-1}+j_{s}\right),
$$

where

(9.78)

$$
\Pi\left(j_{1}, \ldots, j_{s}\right)=\left(\frac{1}{\left|n-j_{1}\right|}+\frac{1}{\left|n+j_{2}\right|}\right) \cdots\left(\frac{1}{\left|n-j_{s-1}\right|}+\frac{1}{\left|n+j_{s}\right|}\right) \frac{1}{\left|n-j_{s}\right|} .
$$

By opening the parentheses we get

$$
\Pi\left(j_{1}, \ldots, j_{s}\right)=\sum_{\delta_{1}, \ldots, \delta_{s-1}= \pm 1}\left(\prod_{\nu=1}^{s-1} \frac{1}{\left|n+\delta_{\nu} j_{\nu+\tilde{\delta}_{\nu}}\right|}\right) \frac{1}{\left|n-j_{s}\right|}, \quad \tilde{\delta}_{\nu}=\frac{1+\delta_{\nu}}{2}
$$

Therefore,

$$
\sigma(n, s)=\sum_{\delta_{1}, \ldots, \delta_{s-1}= \pm 1} \tilde{\sigma}\left(\delta_{1}, \ldots, \delta_{s-1}\right)
$$

where

$$
\tilde{\sigma}\left(\delta_{1}, \ldots, \delta_{s-1}\right)=\sum_{j_{1}, \ldots, j_{s} \neq \pm n}\left(\prod_{\nu=1}^{s-1} \frac{1}{\left|n+\delta_{\nu} j_{\nu+\tilde{\delta}_{\nu}}\right|}\right) \frac{1}{\left|n-j_{s}\right|} r\left(n+j_{1}\right) \cdots r\left(j_{s-1}+j_{s}\right) .
$$


In view of $(9.55),(9.57)$ and $(9.80)$, Lemma 47 will be proved if we show that

$$
\tilde{\sigma}\left(\delta_{1}, \ldots, \delta_{s-1}\right) \leq\left(\varepsilon_{n} / 2\right)^{s}, \quad s \geq 2 .
$$

We prove (9.82) by induction in $s$.

If $s=2$ then

$$
\tilde{\sigma}(-1)=\sigma_{1}(n, 2, n) \leq\left(\varepsilon_{n} / 2\right)^{2}
$$

and

$$
\tilde{\sigma}(+1)=\sigma_{2}(n, 2, n) \leq\left(\varepsilon_{n} / 2\right)^{2} .
$$

If $s=3$ then there are four cases:

$$
\begin{gathered}
\tilde{\sigma}(-1,-1)=\sigma_{1}(n, 3, n) \leq\left(\varepsilon_{n} / 2\right)^{3} ; \quad \tilde{\sigma}(+1,+1)=\sigma_{2}(n, 3, n) \leq\left(\varepsilon_{n} / 2\right)^{3} ; \\
\tilde{\sigma}(-1,+1)=\sum_{j_{1} \neq \pm n} \frac{r\left(n+j_{1}\right)}{\left|n-j_{1}\right|} \sum_{j_{2}, j_{3} \neq \pm n} \frac{r\left(j_{1}+j_{2}\right)}{\left|n+j_{3}\right|} \frac{r\left(j_{2}+j_{3}\right)}{\left|n-j_{3}\right|} \\
=\sum_{j_{1} \neq \pm n} \frac{r\left(n+j_{1}\right)}{\left|n-j_{1}\right|} \sigma_{2}\left(n, 2, j_{1}\right) \\
\leq \sigma_{1}(n, 1, n) \cdot \sup _{m} \sigma_{2}(n, 2, m) \leq\|r\| \frac{1}{K}\left(\varepsilon_{n} / 2\right)^{3} \leq\left(\varepsilon_{n} / 2\right)^{3} ; \\
\tilde{\sigma}(+1,-1)=\sum_{j_{1}, j_{2} \neq \pm n} \frac{r\left(n+j_{1}\right) r\left(j_{1}+j_{2}\right)}{\left|n^{2}-j_{2}^{2}\right|} \sum_{j_{3} \neq \pm n} \frac{r\left(j_{2}+j_{3}\right)}{\left|n-j_{3}\right|} \\
\leq \sigma_{2}(n, 2, n) \cdot \sup _{m} \sigma_{1}(n, 1, m) \leq \frac{1}{K}\left(\varepsilon_{n} / 2\right)^{3}\|r\| \leq\left(\varepsilon_{n} / 2\right)^{3} .
\end{gathered}
$$

Next we prove that if (9.82) hold for some $s$, then it holds for $s+2$. Indeed, let us consider the following cases:

(i) $\delta_{s}=\delta_{s+1}=-1$; then we have

$$
\begin{gathered}
\tilde{\sigma}\left(\delta_{1}, \ldots, \delta_{s-1},-1,-1\right)=\sum_{j_{1}, \ldots, j_{s} \neq \pm n}\left(\prod_{\nu=1}^{s-1} \frac{1}{\left|n+\delta_{\nu} j_{\nu+\tilde{\delta}_{\nu}}\right|}\right) \frac{1}{\left|n-j_{s}\right|} \\
\times r\left(n+j_{1}\right) r\left(j_{1}+j_{2}\right) \cdots r\left(j_{s-1}+j_{s}\right) \sum_{j_{s+1}, j_{s+2} \neq \pm n} \frac{r\left(j_{s}+j_{s+1}\right)}{\left|n-j_{s+1}\right|} \frac{r\left(j_{s+1}+j_{s+2}\right)}{\left|n-j_{s+2}\right|} \\
=\sum_{j_{1}, \ldots, j_{s} \neq \pm n}\left(\prod_{\nu=1}^{s-1} \frac{1}{\left|n+\delta_{\nu} j_{\nu+\tilde{\delta}_{\nu}}\right|}\right) \frac{1}{\left|n-j_{s}\right|} r\left(n+j_{1}\right) \cdots r\left(j_{s-1}+j_{s}\right) \sigma_{1}\left(n, 2, j_{s}\right) \\
\leq \tilde{\sigma}\left(\delta_{1}, \ldots, \delta_{s-1}\right) \cdot \sup _{m} \sigma_{1}(n, 2, m) \leq\left(\varepsilon_{n} / 2\right)^{s} \cdot\left(\varepsilon_{n} / 2\right)^{2}=\left(\varepsilon_{n} / 2\right)^{s+2} .
\end{gathered}
$$

(ii) $\delta_{s}=-1, \delta_{s+1}=+1$; then we have

$$
\begin{gathered}
\tilde{\sigma}\left(\delta_{1}, \ldots, \delta_{s-1},-1,+1\right)=\sum_{j_{1}, \ldots, j_{s} \neq \pm n}\left(\prod_{\nu=1}^{s-1} \frac{1}{\left|n+\delta_{\nu} j_{\nu+\tilde{\delta}_{\nu}}\right|}\right) \frac{1}{\left|n-j_{s}\right|} \\
\times r\left(n+j_{1}\right) r\left(j_{1}+j_{2}\right) \cdots r\left(j_{s-1}+j_{s}\right) \sum_{j_{s+1}, j_{s+2} \neq \pm n} \frac{r\left(j_{s}+j_{s+1}\right) r\left(j_{s+1}+j_{s+2}\right)}{\left|n^{2}-j_{s+2}^{2}\right|} \\
\leq \tilde{\sigma}\left(\delta_{1}, \ldots, \delta_{s-1}\right) \cdot \sup _{m} \sigma_{2}(n, 2, m) \leq\left(\varepsilon_{n} / 2\right)^{s} \cdot\left(\varepsilon_{n} / 2\right)^{2}=\left(\varepsilon_{n} / 2\right)^{s+2} .
\end{gathered}
$$

(iii) $\delta_{s}=\delta_{s+1}=+1$; then, if $\delta_{1}=\cdots=\delta_{s-1}=+1$, we have

$$
\tilde{\sigma}\left(\delta_{1}, \ldots, \delta_{s+1}\right)=\sigma_{2}(n, s+2, n) \leq\left(\varepsilon_{n} / 2\right)^{s+2} .
$$


Otherwise, let $\mu<s$ be the largest index such that $\delta_{\mu}=-1$. Then we have

$$
\begin{gathered}
\tilde{\sigma}\left(\delta_{1}, \ldots, \delta_{s-1},+1,+1\right)=\sum_{j_{1}, \ldots, j_{\mu} \neq \pm n}\left(\prod_{\nu=1}^{\mu-1} \frac{1}{\left|n+\delta_{\nu} j_{\nu+\tilde{\delta}_{\nu}}\right|}\right) \frac{1}{\left|n-j_{\mu}\right|} \\
\times r\left(n+j_{1}\right) r\left(j_{1}+j_{2}\right) \cdots r\left(j_{\mu-1}+j_{\mu}\right) \sigma_{2}\left(n, s+2-\mu, j_{\mu}\right) \\
\leq \tilde{\sigma}\left(\delta_{1}, \ldots, \delta_{\mu-1}\right) \cdot \sup _{m} \sigma_{2}\left(n, s+2-\mu, j_{\mu}\right) \leq\left(\varepsilon_{n} / 2\right)^{\mu} \cdot\left(\varepsilon_{n} / 2\right)^{s+2-\mu}=\left(\varepsilon_{n} / 2\right)^{s+2} . \\
\quad(\text { iv }) \delta_{s}=+1, \delta_{s+1}=-1 ; \text { then, if } \delta_{1}=\cdots=\delta_{s-1}=+1, \text { we have } \\
\quad \sum_{j_{1}, \ldots, j_{s+1} \neq \pm n}\left(\prod_{\nu=1}^{s} \frac{1}{\left|n+\delta_{\nu+1}\right|}\right) \frac{1}{\left.\mid n-\delta_{s+1},-1\right)=\tilde{\sigma}(+1, \ldots,+1,-1,-1)=} \\
\leq \sigma_{2}(n, s+1, n) \cdot \sup _{m} \sigma_{1}(n, 1, m) \leq \frac{1}{K}\left(\varepsilon_{n} / 2\right)^{s+2} \cdot\|r\| \leq\left(\varepsilon_{n} / 2\right)^{s+2} .
\end{gathered}
$$

Otherwise, let $\mu<s$ be the largest index such that $\delta_{\mu}=-1,1 \leq \mu<n$. Then we have

$$
\begin{gathered}
\tilde{\sigma}\left(\delta_{1}, \ldots, \delta_{s-1},+1,-1\right)=\sum_{j_{1}, \ldots, j_{\mu} \neq \pm n}\left(\prod_{\nu=1}^{\mu-1} \frac{1}{\left|n+\delta_{\nu} j_{\nu+\tilde{\delta}_{\nu}}\right|}\right) \frac{1}{\left|n-j_{\mu}\right|} \\
\times \sum_{j_{\mu+1}, \ldots, j_{s+1} \neq \pm n} \frac{r\left(j_{\mu}+j_{\mu+1}\right)}{\left|n+j_{\mu+2}\right|} \cdots \frac{r\left(j_{s-1}+j_{s}\right)}{\left|n+j_{s+1}\right|} \frac{r\left(j_{s}+j_{s+1}\right)}{\left|n-j_{s+1}\right|} \sum_{j_{s+2} \neq \pm n} \frac{r\left(j_{s+1}+j_{s+2}\right)}{\left|n-j_{s+2}\right|} \\
\leq \tilde{\sigma}\left(\delta_{1}, \ldots, \delta_{\mu-1}\right) \cdot \sup _{m} \sigma_{2}(n, s+1-\mu, m) \cdot \sup _{k} \sigma_{1}(n, 1, k) \\
\leq\left(\varepsilon_{n} / 2\right)^{\mu} \cdot \frac{1}{K}\left(\varepsilon_{n} / 2\right)^{s+2-\mu}\|r\| \leq\left(\varepsilon_{n} / 2\right)^{s+2} .
\end{gathered}
$$

Hence (9.82) holds for $s \geq 2$.

Now (9.57), (9.80) and (9.82) imply (9.55), which completes the proof of Lemma 47 .

Now we are ready to accomplish the proof of Theorem 45.

9.4. Proof of the main theorem on the deviations $\left\|P_{n}-P_{n}^{0}\right\|_{L^{1} \rightarrow L^{\infty}}$. We need - because we want to use (9.14) - to give estimates of $A(n, s)$ from (9.15), or (9.26). By (9.31) and (9.32), we reduce such estimates to analysis of quantities $A_{j}(n, s), j=1, \ldots, 7$.

With $\rho_{n} \in(9.40)$ and $\varepsilon_{n} \in(9.72)$, we set

$$
\kappa_{n}=\max \left\{\rho_{n}, \varepsilon_{n}\right\} .
$$

Then, by Lemma 47 (and Corollary 48), i.e., by the inequality (9.56), we have (in view of $(9.42),(9.45),(9.48)$ and $(9.50)-(9.53))$ the following estimates for $A_{j}$ :

$$
\begin{gathered}
A_{1} \leq 4 \kappa_{n}^{s+1}, \quad A_{j} \leq 2^{s+1} \cdot 2 \kappa_{n}^{s+1}, \quad j=2,4 \\
A_{j} \leq 2^{s+1} \sum_{\nu=1}^{s}\left(2 \kappa_{n}^{\nu} \cdot \kappa_{n}^{s-\nu+1}\right)=s 2^{s+2} \kappa_{n}^{s+1}, \quad j=3,5
\end{gathered}
$$




$$
\begin{gathered}
A_{6} \leq 2^{s+1} \sum_{\nu=1}^{s}\left(\kappa_{n}^{\nu} \cdot \kappa_{n}^{s-\nu+1}\right)=s 2^{s+1} \kappa_{n}^{s+1} \\
A_{7} \leq 2^{s} \sum_{1 \leq \nu+\mu \leq s}\left(4 \kappa_{n}^{\nu} \cdot \kappa^{\mu-\nu} \cdot \kappa_{n}^{s-\mu+1}\right)=s(s-1) 2^{s+1} \kappa_{n}^{s+1} .
\end{gathered}
$$

In view of (9.22), (9.72) and (9.32), these inequalities imply

$$
A(n, s) \leq(2+s)^{2}\left(2 \kappa_{n}\right)^{s+1} .
$$

Therefore, the right-hand side of (9.14) does not exceed

$$
\sum_{s=0}^{\infty} A(n, s) \leq\left(4 \kappa_{n}\right) \sum_{s=0}^{\infty}(s+1)(s+2)\left(2 \kappa_{n}\right)^{s}=\frac{8 \kappa_{n}}{\left(1-2 \kappa_{n}\right)^{3}} .
$$

Therefore, if $\kappa_{n}<1 / 4$ (which holds for $n \geq N^{*}$ with a proper choice of $N^{*}$ ), then $\sum_{s=0}^{\infty} A(n, s) \leq 64 \kappa_{n}$. Thus, by (9.14) and the notations (9.9),

$$
\left\|P_{n}-P_{n}^{0}\right\|_{L^{1} \rightarrow L^{\infty}} \leq \sum_{k, m}\left|B_{k m}(n)\right| \leq 64 \kappa_{n}, \quad n \geq N^{*},
$$

where $\kappa_{n} \in(9.83)$.

This completes the proof of Theorem 45. Of course, Proposition 44 follows because $\|T\|_{L^{2} \rightarrow L^{\infty}} \leq\|T\|_{L^{1} \rightarrow L^{\infty}}$ for any well defined operator $T$.

9.5. Miscellaneous. As we have already noticed many times, Theorem 45 (or Proposition 44) is an essential step in the proof of our main result Theorem 29 (see an announcement in [15], Theorem 9, or [16], Theorem 23) about the relationship between the rate of decay of spectral gap sequences (and deviations) and the smoothness of the potentials $v$ under the a priori assumption that $v$ is a singular potential, i.e., that $v \in H_{P e r}^{-1}$. But Theorem 45 is important outside this context as well. Now we will mention one very important corollary.

THEOREM 51. In the above notations, the $L^{p}$-norms, $1 \leq p \leq \infty$, on Riesz subspaces $E^{N}=\operatorname{Ran} S_{N}$, and $E_{n}=\operatorname{Ran} P_{n}, n \geq N$, are uniformly equivalent; more precisely,

$$
\|f\|_{\infty} \leq 3\|f\|_{1}, \quad \forall f \in E_{n}, \quad n \geq N^{*}(v),
$$

and

$$
\|f\|_{1} \leq\|f\|_{\infty} \leq C(N)\|f\|_{1}, \quad \forall f \in E^{N}
$$

where

$$
C(N) \leq 50 N \log N .
$$

Proof. By (9.8), if $N$ is large enough,

$$
\left\|P_{n}-P_{n}^{0}\right\|_{L^{1} \rightarrow L^{\infty}} \leq \frac{1}{2}, \quad n \geq N .
$$

If we are more careful when using $(9.83),(9.84),(9.40)$ and $(9.72)$, we may claim (9.88) for $N$ such that

$$
2^{9}(1+\|r\|)\left(\mathcal{E}_{\sqrt{N}}(r)+\frac{2}{N^{1 / 4}}\left(\|r\|^{1 / 2}+(\log 6 N)^{1 / 4}\right)\right) \leq \frac{1}{2} .
$$

If $f \in E_{n}, n \geq N$, we have

$$
f=P_{n} f=\left(P_{n}-P_{n}^{0}\right) f+P_{n}^{0} f
$$


where, for $b c=P e r^{ \pm}$

$$
P_{n}^{0} f=f_{n} e^{i n x}+f_{-n} e^{-i n x}, \quad f_{k}=\frac{1}{\pi} \int_{0}^{\pi} f(x) e^{-i k x} d x,
$$

and, for $b c=D i r$,

$$
P_{n}^{0} f=2 g_{n} \sin n x, \quad g_{n}=\frac{1}{\pi} \int_{0}^{\pi} f(x) \sin n x d x .
$$

In either case $\left\|P_{n} f\right\|_{\infty} \leq 2\|f\|_{1}$, and therefore, if $\|f\|_{1} \leq 1$ we have

$$
\|f\|_{\infty} \leq\left\|\left(P_{n}-P_{n}^{0}\right) f\right\|_{\infty}+\left\|P_{n}^{0} f\right\|_{\infty} \leq 1 / 2+2 \leq 3 .
$$

This proves (9.85).

Recall that the projection

$$
S_{N}=\frac{1}{2 \pi i} \int_{\partial R_{N}}\left(z-L_{b c}\right)^{-1} d z
$$

where, as in (5.40), [16],

$$
R_{N}=\left\{z \in \mathbb{C}:-N<\operatorname{Re} z<N^{2}+N,|\operatorname{Im} z|<N\right\},
$$

is finite-dimensional (see [16], (5.54), (5.56), (5.57) for $\left.\operatorname{dim} S_{N}\right)$. Now we follow the inequalities proven in [16] to explain (9.86) and (9.87). Lemma 20, inequality (5.41) in $[\mathbf{1 6}]$, states that

$$
\sup \left\{\left\|K_{\lambda} V K_{\lambda}\right\|_{H S}: \lambda \notin R_{N}, \operatorname{Re} \lambda \leq N^{2}-N\right\} \leq C\left(\frac{(\log N)^{1 / 2}}{N^{1 / 4}}\|q\|+\mathcal{E}_{4 \sqrt{N}}(q)\right) .
$$

But by (9.94)

$$
S_{N}-S_{N}^{0}=\frac{1}{2 \pi i} \int_{\Gamma} K_{\lambda} \sum_{m=1}^{\infty}\left(K_{\lambda} V K_{\lambda}\right)^{m} K_{\lambda} d \lambda,
$$

where we can choose $\Gamma$ to be the boundary $\partial \Pi$ of the rectangle

$$
\Pi(H)=\left\{z \in \mathbb{C}:-H \leq \operatorname{Re} z \leq N^{2}+N,|\operatorname{Im} z| \leq H\right\}, \quad H \geq N .
$$

Then by (9.96) and (9.97) the norm of the sum in the integrand can be estimated by

$$
\left\|\sum_{1}^{\infty}\right\|_{2 \rightarrow 2} \leq \sum_{1}^{\infty}\left\|K_{\lambda} V K_{\lambda}\right\|_{H S}^{m} \leq 1, \quad \forall \lambda \in \partial \Pi(H)
$$

if (compare with (9.89)) $N \geq N^{*}(q)$ and $N^{*}=N^{*}(q)$ is chosen to guarantee that

$$
\text { "the right side in }(9.96) \text { " } \leq 1 / 2 \text { for } N \geq N^{*} \text {. }
$$

The additional factor $K_{\lambda}$ is a multiplier operator defined by the sequence $\tilde{K}=$ $\left\{1 / \sqrt{\lambda-k^{2}}\right\}$, so its norms $\left\|K_{\lambda}: L^{1} \rightarrow L^{2}\right\|$ and $\left\|K_{\lambda}: L^{2} \rightarrow L^{\infty}\right\|$ are estimated by $2 \tilde{\kappa}$, where

$$
\tilde{\kappa}=\left\|\tilde{K}_{\lambda}: \ell^{\infty} \rightarrow \ell^{2}\right\|=\left\|\tilde{K}_{\lambda}: \ell^{2} \rightarrow \ell^{1}\right\|=\sum_{k} \frac{1}{\left|\lambda-k^{2}\right|} .
$$

Therefore, by (9.99) and (9.101),

$$
\alpha(\lambda):=\left\|K_{\lambda}\left(\sum_{1}^{\infty}\left(K_{\lambda} V K_{\lambda}\right)^{m}\right) K_{\lambda}: L^{1} \rightarrow L^{\infty}\right\| \leq \sum_{k} \frac{4}{\left|\lambda-k^{2}\right|} .
$$


By Lemma 18(a) in [16] (or, Lemma 79(a) in [14])

$$
\sum_{k} \frac{1}{\left|n^{2}-k^{2}\right|+b} \leq C_{1} \frac{\log b}{\sqrt{b}} \text { if } n \in \mathbb{N}, b \geq 2 .
$$

(In what follows $C_{j}, j=1,2, \ldots$ are absolute constants; $C_{1} \leq 12$.) These inequalities are used to estimate the norm $\alpha(\lambda)$ on the boundary $\partial \Pi(H)=\cup I_{k}(H), k=$ $1,2,3,4$, where

$$
\begin{gathered}
I_{1}(H)=\{z: \operatorname{Re} z=-H,|\operatorname{Im} z| \leq H\} \\
I_{2}(H)=\left\{z:-H \leq \operatorname{Re} z \leq N^{2}+N, \operatorname{Im} z=H\right\} \\
I_{3}(H)=\left\{z: \operatorname{Re} z=N^{2}+N,|\operatorname{Im} z| \leq H\right\} \\
I_{2}(H)=\left\{z:-H \leq \operatorname{Re} z \leq N^{2}+N, \operatorname{Im} z=-H\right\}
\end{gathered}
$$

Then we get

$$
\begin{gathered}
\int_{I_{1}} \alpha(\lambda)|d \lambda| \leq C_{2} \frac{\log H}{\sqrt{H}} \cdot H \\
\int_{I_{k}} \alpha(\lambda)|d \lambda| \leq C_{3} \frac{\log H}{\sqrt{H}} \cdot N^{2}, \quad k=2,4 . \\
\int_{I_{3}} \alpha(\lambda)|d \lambda| \leq C_{4} \int_{0}^{H} \frac{\log (N+y)}{\sqrt{N+y}} d y \leq C_{5} \sqrt{H} \log H .
\end{gathered}
$$

If we put $H=N^{2}$ and sum up these inequalities we get by (9.97)

$$
\left\|S_{N}-S_{N}^{0}\right\|_{L^{1} \rightarrow L^{\infty}} \leq C_{6} N \log N
$$

where $C_{6}$ is an absolute constant $\leq 600$.

Now, as in (9.90) and (9.91), let us notice that for $g \in E^{N}$

$$
g=S_{N} g=\left(S_{N}-S_{N}^{0}\right) g+S_{N}^{0} g
$$

where

(9.106) $\quad S_{N}^{0} g=\sum_{|k| \leq N} g_{k} e^{i k x}, \quad k$ even for $b c=P e r^{+}$, odd for $b c=P e r^{-}$,

and

$$
S_{N}^{0} g=2 \sum_{|k| \leq N} \tilde{g}_{k} \sin k x, \quad b c=D i r
$$

where

$$
g_{k}=\frac{1}{\pi} \int_{0}^{\pi} g(x) e^{i k x} d x, \quad \tilde{g}_{k}=\frac{1}{\pi} \int_{0}^{\pi} g(x) \sin k x d x .
$$

In either case

$$
\left\|S_{N}^{0} g\right\|_{\infty} \leq 2 N\|g\|_{1} .
$$

Therefore, by (9.104) and (9.109), if $\|f\|_{1} \leq 1$ we have

$$
\|f\|_{\infty} \leq C_{6} N \log N+2 N \leq C_{7} N \log N, \quad N \geq N^{*} \in(9.100) .
$$




\section{References}

[1] J. Adduci, P. Djakov, B. Mityagin, Convergence radii of eigenvalues of tri-diagonal matrices, manuscript, arXiv: 0901.4031.

[2] S. Albeverio, F. Gesztesy, R. Hegh-Krohn, H. Holden, Solvable models in quantum mechanics. Texts and Monographs in Physics. Springer-Verlag, New York, 1988.

[3] S. Albeverio and P. Kurasov, Singular perturbations of differential operators. Solvable Schrödinger type operators. London Mathematical Society Lecture Note Series, 271. Cambridge University Press, Cambridge, 2000.

[4] F.V. Atkinson, Discrete and continuous boundary problems, Academic Press, New York, 1964.

[5] Dzh.-G. Bak and A. A. Shkalikov, Multipliers in dual Sobolev spaces and Schrödinger operators with distribution potentials. (Russian) Mat. Zametki 71 (2002), no. 5, 643-651; translation in Math. Notes 71 (2002), no. 5-6, 587-594.

[6] F. A. Berezin and L. D. Faddeev, Remark on the Schrödinger equation with singular potential (Russian), Dokl. Akad. Nauk SSSR 137 (1961), 1011-1014; English transl., Soviet Math. Dokl. 2 (1961), 372-375.

[7] F. A. Berezin, On the Lee model. (Russian) Mat. Sb. 60 (1963) 425-446; English transl., Amer. Math. Soc. Transl. (2) 56 (1966), 249-272.

[8] N. Dernek and O. A. Veliev, On the Riesz basisness of the root functions of the nonself-adjoint Sturm-Liouville operator. Israel J. Math. 145 (2005), 113-123.

[9] P. Djakov and B. Mityagin, Smoothness of Schrödinger operator potential in the case of Gevrey type asymptotics of the gaps, J. Funct. Anal. 195 (2002), 89-128.

[10] P. Djakov and B. Mityagin, Spectral gaps of the periodic Schrödinger operator when its potential is an entire function. Adv. in Appl. Math. 31 (2003), no. 3, 562-596.

[11] P. Djakov and B. Mityagin, Spectral triangles of Schrödinger operators with complex potentials. Selecta Math. (N.S.) 9 (2003), 495-528.

[12] P. Djakov and B. Mityagin, Instability zones of a periodic 1D Dirac operator and smoothness of its potential. Comm. Math. Phys. 259 (2005), 139-183.

[13] P. Djakov and B. Mityagin, Trace formula and spectral Riemann surfaces for a class of tridiagonal matrices, J. Approx. Theory 139 (2006), 293-326.

[14] P. Djakov and B. Mityagin, Instability zones of periodic 1D Schrödinger and Dirac operators, in Russian, Uspekhi Mat. Nauk 61:4 (2006), 77-182; Enlish translation: Russian Math. Surveys 61:4 (2006), 663-766.

[15] P. Djakov and B. Mityagin, Spectral gap asymptotics of one dimensional Schrödinger operators with singular periodic potentials, Integral Transforms and Special Functions 20 (2009), 265-273.

[16] P. Djakov and B. Mityagin, Fourier method for one dimensional Schrödinger operators with singular periodic potentials, manuscript, arXiv:0710.0237, to appear in Topics in Operator Theory, editors: J. A. Ball, V. Bolotnikov, J. W. Helton, L Rodman, I. M. Spitkovsky, (series Operator Theory: Advances and Applications), Birkhuser Verlag, Basel, 2009.

[17] P. Djakov and B. Mityagin, Bari-Markus property for Riesz projections of Hill operators with singular potentials, Contemporary Mathematics 481, 59-80 (2009), AMS-Functional Analysis and Complex Analysis, editors A. Aytuna, R. Meize, T. Terzioglu, D. Vogt.

[18] P. Djakov and B. Mityagin, Deviations of Riesz projections of 1D Dirac operators, arXiv: 0901.0856

[19] P. Djakov and B. Mityagin, Convergence of spectral expansions of 1D Dirac operators subject to regular boundary conditions, in preparation.

[20] B. A. Dubrovin, The inverse problem of scatering theory for periodic finite-zone potentials, Funktsional. Anal. i Prilozhen. 9 (1975), 65-66.

[21] B. A. Dubrovin, V. A. Matveev, S. P. Novikov, Nonlinear equations of Korteweg-de Vries type, finite-band linear operators and Abelian varieties. (Russian) Uspehi Mat. Nauk 31 (1976), 55-136.

[22] M. S. P. Eastham, The spectral theory of periodic differential operators, Hafner, New York 1974.

[23] W. N. Everitt and A. Zettl, Generalized symmetric ordinary differential expressions. I. The general theory. Nieuw Arch. Wisk. (3) 27 (1979), 363-397. 
[24] W. N. Everitt and A. Zettl, A. Sturm-Liouville differential operators in direct sum spaces. Rocky Mountain J. Math. 16 (1986), 497-516.

[25] M. G. Gasymov, Spectral analysis of a class of second order nonselfadjoint differential operators, Functional Anal. and its Appl. 14 (1980), 14-19.

[26] I. M. Gelfand and B. M. Levitan, On the determination of a differential equation by its spectral function. (Russian) Doklady Akad. Nauk SSSR (N.S.) 77 (1951), 557-560.

[27] F. Gesztesy and V. Tkachenko, A criterian for a Hill operator to be a spectral operator, Journal D'Analyse Mathématicue, 107 (2009), 287-353.

[28] I. C. Gohberg, M. G. Krein, Introduction to the theory of linear non-self-adjoint operators, vol. 18 (Translation of Mathematical Monographs). Providence, Rhode Island, American Mathematical Society 1969.

[29] B. Grébert, T. Kappeler and B. Mityagin, Gap estimates of the spectrum of the ZakharovShabat system, Appl. Math. Lett. 11 (1998), 95-97.

[30] B. Grébert and T. Kappeler, Estimates on periodic and Dirichlet eigenvalues for the ZakharovShabat system, Asymptotic Analysis 25 (2001), 201-237; Erratum: "Estimates on periodic and Dirichlet eigenvalues for the Zakharov-Shabat system" Asymptot. Anal. 29 (2002), no. $2,183$.

[31] B. Grébert, T. Kappeler, Density of finite gap potentials for the Zakharov-Shabat system. Asymptot. Anal. 33 (2003), 1-8.

[32] H. Hochstadt, Estimates on the stability intervals for the Hill's equation, Proc. AMS 14 (1963), 930-932.

[33] H. Hochstadt, On the determination of a Hill's equation from its spectrum, Arch. Rational Mech. Anal. 19 (1965), 353 - 362

[34] R. O. Hryniv and Ya. V. Mykytyuk, 1-D Schrödinger operators with periodic singular potentials. Methods Funct. Anal. Topology 7 (2001), 31-42.

[35] R. O. Hryniv and Ya. V. Mykytyuk, 1-D Schrödinger operators with singular Gordon potentials. Methods Funct. Anal. Topology 8 (2002), 36-48.

[36] R. O. Hryniv and Ya. V. Mykytyuk, Inverse spectral problems for Sturm-Liouville operators with singular potentials. Inverse Problems 19 (2003), 665-684.

[37] R. O. Hryniv and Ya. V. Mykytyuk, Transformation operators for Sturm-Liouville operators with singular potentials. Math. Phys. Anal. Geom. 7 (2004), 119-149.

[38] R. O. Hryniv and Ya. V. Mykytyuk, Inverse spectral problems for Sturm-Liouville operators with singular potentials. IV. Potentials in the Sobolev space scale. Proc. Edinb. Math. Soc. 49 (2006), 309-329.

[39] R. O. Hryniv and Ya. V. Mykytyuk, Eigenvalue asymptotics for Sturm-Liouville operators with singular potentials, J. Funct. Anal. 238 (2006), 27-57.

[40] T. Kappeler and B. Mityagin, Gap estimates of the spectrum of Hill's Equation and Action Variables for KdV, Trans. AMS 351 (1999), 619-646.

[41] T. Kappeler and B. Mityagin, Estimates for periodic and Dirichlet eigenvalues of the Schrödinger operator, SIAM J. Math. Anal. 33 (2001), 113-152.

[42] T. Kappeler and C. Möhr, Estimates for periodic and Dirichlet eigenvalues of the Schrödinger operator with singular potential, J. Funct. Anal. 186 (2001), 69-91.

[43] T. Kappeler, J. Pöschel, Spectral gaps of potentials in weighted Sobolev spaces, In: W. Craig (ed), Hamiltonian Systems and Applications, Springer, 2008, 421-430.

[44] T. Kato, Perturbation theory for linear operators, Springer Verlag, Berlin, 1980.

[45] E. Korotyaev, Characterization of the spectrum of Schroedinger operators with periodic distributions, Int. Math. Res. Not. 37 (2003), 2019 - 2031.

[46] E. Korotyaev, Estimates for the Hill operator. II. J. Differential Equations 223 (2006), no. 2, 229-260.

[47] E. Korotyaev, A priori estimates for the Hill and Dirac operators, arXiv:math.SP/0701437v1 16 Jan 2007

[48] M. G. Krein, On a generalization of investigations of Stielstjes, Dokl. Akad. Nauk SSSR, 87 (1952), 881-884.

[49] M. G. Krein, On the characteristic function $A(\lambda)$ of a linear canonical system of system differential equation of the second order with periodic coefficients, Prikl. Mat. Mekh. 21 (1957), 320-329.

[50] P. Kuchment, Floquet theory for partial differential equations Basel-Boston, Birkhauser Verlag, 1993. 
[51] P. Kurasov, On the Coulomb potential in dimension one J. Phys. A: Math. Gen., 29 (1996), $1767-1771$.

[52] W. Magnus and S. Winkler, "Hill's equation", Interscience Publishers, John Wiley, 1969.

[53] V. A. Marchenko and I. V. Ostrovskii, Characterization of the spectrum of Hill's operator, Matem. Sborn. 97 (1975), 540-606; English transl. in Math. USSR-Sb. 26 (175).

[54] H. McKean and E. Trubowitz, Hill's operator and hyperelliptic function theory in the presence of infinitely many branch points. Comm. Pure Appl. Math. 29 (1976), 143-226.

[55] J. Meixner and F. W. Schäfke, Mathieusche Funktionen und Sphäroidfunktionen, Springer Verlag, 1954.

[56] J. Meixner, F. W. Schäfke and G. Wolf, Mathieu Functions and Spheroidal Functions and their Mathematical Foundations, Lecture Notes in Math. 837, Springer Verlag, 1980.

[57] R. A. Minlos and L. D. Faddeev, On the point interaction for a three-particle system in quantum mechanics, Dokl. Akad. Nauk SSSR 141 (1961), 1335-1338 (Russian); translated as Soviet Physics Dokl. 6 (1962), 1072-1074.

[58] B. Mityagin, Manuscript, 2000.

[59] B. Mityagin, Convergence of expansions in eigenfunctions of the Dirac operator. (Russian) Dokl. Akad. Nauk 393 (2003), no. 4, 456-459.

[60] B. Mityagin, Spectral expansions of one-dimensional periodic Dirac operators. Dyn. Partial Differ. Equ. 1 (2004), 125-191.

[61] M. A. Naimark, Linear differential operators, Moscow, 1969.

[62] M. I. Neı̌man-zade and A. A. Shkalikov, Schrödinger operators with singular potentials from spaces of multipliers. (Russian) Mat. Zametki 66 (1999), no. 5, 723-733; translation in Math. Notes 66 (1999), no. 5-6, 599-607

[63] M. I. Neľman-zade and A. A. Shkalikov, Schrödinger operators with singular potentials. (Russian) Spectral and evolution problems, Vol. 11 (Sevastopol, 2000), 3-8, Natl. Taurida Univ. "V. Vernadsky", Simferopol, 2001.

[64] J. Pöschel, Hill's potentials in weighted Sobolev spaces and their spectral gaps, Stuttgarter Mathematische Berichte, Preprint 2004/12, August 2004, pp. 1-28.

[65] J. Pöschel, Spectral gaps of potentials in weighted Sobolev spaces. In: W. Craig (ed), Hamiltonian Systems and Applications, Springer, 2008, 421-430.

[66] J. J. Sansuc and V. Tkachenko, Spectral parametrization of non-selfadjoint Hill's operators, J. Differential Equations 125 (1996), 366-384.

[67] J. J. Sansuc and V. Tkachenko, Spectral properties of non-selfadjoint Hill's operators with smooth potentials, in A. Boutet de Monvel and V. Marčenko (eds.), "Algebraic and geometric methods in mathematical physics", 371-385, Kluver, 1996.

[68] A. M. Savchuk, On the eigenvalues and eigenfunctions of the Sturm-Liouville operator with a singular potential (Russian) Mat. Zametki 69 (2001), no. 2, 277-285; translation in Math. Notes 69 (2001), no. 1-2, 245-252

[69] A. M. Savchuk and A. A. Shkalikov, Sturm-Liouville operators with singular potentials. (Russian) Mat. Zametki 66 (1999), no. 6, 897-912; translation in Math. Notes 66 (1999), no. 5-6, $741-753$.

[70] A. M. Savchuk and A. A. Shkalikov, A trace formula for Sturm-Liouville operators with singular potentials. (Russian) Mat. Zametki 69 (2001), 427-442; translation in Math. Notes 69 (2001), 387-400.

[71] Savchuk, A. M.; Shkalikov, A. A. Sturm-Liouville operators with distribution potentials. (Russian) Tr. Mosk. Mat. Obs. 64 (2003), 159-212; translation in Trans. Moscow Math. Soc. 2003, 143-192

[72] A. M. Savchuk and A. A. Shkalikov, On the eigenvalues of the Sturm-Liouville operator with potentials in Sobolev spaces, (Russian), Mat. Zametki 80 (2006), 864-884.

[73] A. A. Shkalikov and O. A. Veliev, On the Riesz Basis Property of the Eigen- and Associated Functions of Periodic and Antiperiodic Sturm-Liouville Problems, arXiv:0811.2337, 16 pages.

[74] V. Tkachenko, Spectral analysis of the nonselfadjoint Hill operator, (Russian) Dokl. Akad. Nauk SSSR 322 (1992), 248-252; translation in Soviet Math. Dokl. 45 (1992), 78-82.

[75] V. Tkachenko, Discriminants and generic spectra of nonselfadjoint Hill's operators, Spectral operator theory and related topics, 41-71, Adv. Soviet Math., 19, Amer. Math. Soc., Providence, RI, 1994.

[76] E. Trubowitz, The inverse problem for periodic potentials, CPAM 30 (1977), 321-342. 
[77] O. A. Veliev and M. T. Duman, The spectral expansion for a nonself-adjoint Hill operator with a locally integrable potential. J. Math. Anal. Appl. 265 (2002), no. 1, 76-90.

[78] H. Volkmer, Quadratic growth of convergence radii for eigenvalues of two-parameter SturmLiouville equations, J. Differential Equations 128 (1996), no. 1, 327-345.

[79] H. Volkmer, On the growth of convergence radii for the eigenvalues of the Mathieu equation. Math. Nachr. 192 (1998), 239-253.

[80] H. Volkmer, Asymptotics of convergence radii for the two-parameter Airy equation. Differential equations and dynamical systems (Waterloo, ON, 1997). Dynam. Contin. Discrete Impuls. Systems 5 (1999), no. 1-4, 31-38.

[81] H. Volkmer, Convergence radii for eigenvalues of two-parameter Sturm-Liouville problems. Analysis (Munich) 20 (2000), no. 3, 225-236.

[82] H. Volkmer, On Riemann surfaces of analytic eigenvalue functions. Complex Var. Theory Appl. 49 (2004), no. 3, 169-182.

[83] J. Weidmann, Spectral theory of ordinary differential operators, Lect. Notes in Math. 1258, Springer, Berlin, 1987

Sabanci University, Orhanli, 34956 Tuzla, Istanbul, Turkey

E-mail address: djakov@sabanciuniv.edu

Department of Mathematics, The Ohio State University, 231 West 18th Ave, ColumBUS, OH 43210, USA

E-mail address: mityagin.1@osu.edu 